Christian Nassau

\title{
Ein neuer Algorithmus zur Untersuchung der Kohomologie der Steenrod-Algebra
}





\title{
Ein neuer Algorithmus zur Untersuchung der Kohomologie der Steenrod-Algebra
}

\author{
Dissertation \\ zur Erlangung des Doktorgrades \\ der Naturwissenschaften
}

vorgelegt beim Fachbereich Mathematik

der Johann Wolfgang Goethe-Universität

in Frankfurt am Main

\author{
von Christian Nassau \\ aus Bad Homburg
}

Frankfurt am Main

2001

(DF 1) 
Die Deutsche Bibliothek - CIP-Einheitsaufnahme Nassau, Christian:

Ein neuer Algorithmus zur Untersuchung der Kohomologie der Steenrod-Algebra / von Christian Nassau. - Berlin : Logos-Verl., 2002

Zugl.: Frankfurt (Main), Univ., Diss., 2001

ISBN 3-89722-881-5

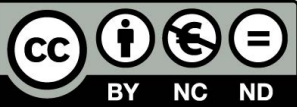

(C) Copyright Logos Verlag Berlin 2002

Alle Rechte vorbehalten.

ISBN 3-89722-881-5

Logos Verlag Berlin

Comeniushof, Gubener Str. 47,

10243 Berlin

Tel.: +4903042851090

Fax: +4903042851092

INTERNET: http://www.logos-verlag.de 


\section{Zusammenfassung}

Gegenstand der Arbeit ist die Untersuchung der Kohomologie der Steenrod-Algebra, hauptsächlich zur Primzahl $p=2$. Vorgestellt wird ein Lemma, das sowohl die computerunterstützte Berechnung, wie auch die anschliessende Untersuchung einer minimalen Auflösung dieser Algebra vereinfacht. Der theoretische Hintergrund dieses Lemmas ist in der für Kohomologiefragen seit langem bekannten Bedeutung elementar-abelscher Untergruppen zu suchen, die hier in der Gestalt der internen Margolis-Differentiale der Algebra auftreten. Aus diesem Hintergrund wird in der vorliegenden Arbeit ein Algorithmus gewonnen, der das Rechnen in bisher unerreichbaren Dimensionen erlaubt: mit vertretbarem Rechenaufwand erhalten wir eine minimale Auflösung, die bis zur Dimension 210 vollständig ist. Vorher war nur circa die Hälfte dieses Bereichs bekannt.

Das Interesse an diesen Kohomologiegruppen kommt aus der Topologie, und näherhin aus der stabilen Homotopietheorie: Die Steenrod-Algebra kann man am einfachsten als Endomorphismenring des Eilenberg-MacLane-Spektrums $H \mathbb{F}_{p}$, dh. des Systems der Eilenberg-MacLane-Räume $\left\{K\left(\mathbb{F}_{p}, n\right)\right\}_{n=0,1,2, \ldots}$, definieren. Als solcher operiert sie auf natürliche Weise auf allen Kohomologiegruppen $H^{*}\left(-; \mathbb{F}_{p}\right)$. Mithilfe der Adams-Spektralreihe

$$
\operatorname{Ext}_{A}^{s, t}\left(H^{*}\left(Y ; \mathbb{F}_{p}\right), H^{*}\left(X ; \mathbb{F}_{p}\right)\right) \Rightarrow\left\{\Sigma^{t-s} X, Y\right\}_{(p)}
$$

gewinnt man so hoch strukturierte obere Schranken für die $p$-Lokalisierungen der stabilen Homotopiegruppen

$$
\left\{\Sigma^{k} X, Y\right\}=\lim _{\rightarrow}\left[\Sigma^{k+n} X, \Sigma^{n} Y\right], \quad(X \text { kompakt }) .
$$

Der hier zumeist beschworene Fall $X=Y=S^{0}$ führt auf der linken Seite zu $\operatorname{Ext}_{A}^{s, t}\left(\mathbb{F}_{p}, \mathbb{F}_{p}\right)$, was auch schlicht als Kohomologie der Steenrod-Algebra bezeichnet wird. Diese ist also der Ausgangsterm einer Spektralreihe, die die $p$-Lokalisierung der stabilen Homotopiegruppen der Sphäre(n)

$$
\pi_{k}^{\text {stab }}(S)=\lim _{\rightarrow} \pi_{n+k}\left(S^{n}\right)=\left\{S^{k}, S^{0}\right\}
$$

berechnet.

Wir zeigen im weiteren, wie aus der minimalen Auflösung mit vergleichsweise geringem Aufwand weitere Informationen gewonnen werden können: darunter fällt die Berechnung von $\operatorname{Ext}_{A}^{s, t}(M, N)$ für kleine endliche $A$-Moduln $M$ und $N$, die Berechnung der Mahowald'schen Wurzelinvarianten, die damit eng verwandte Bestimmung der Verdopplungsoperation $\mathrm{Sq}^{0}: \operatorname{Ext}_{A}^{s, t}\left(\mathbb{F}_{2}, \mathbb{F}_{2}\right) \rightarrow \operatorname{Ext}_{A}^{s, 2 t}\left(\mathbb{F}_{2}, \mathbb{F}_{2}\right)$, und die Berechnung der algebraischen Hurewicz-Abbildungen $\operatorname{Ext}_{A}^{s, t}\left(\mathbb{F}_{2}, \mathbb{F}_{2}\right) \rightarrow \operatorname{Ext}_{A(n)}^{s, t}\left(\mathbb{F}_{2}, \mathbb{F}_{2}\right)$. Für $n=1$ und $n=2$ modellieren diese algebraisch die topologischen HurewiczTransformationen

$$
\begin{aligned}
& \pi_{*}^{\text {stab }}(S) \rightarrow \pi_{*}(\mathrm{ko}) \\
& \text { (Vergleich mit } K \text {-Theorie), } \\
& \pi_{*}^{\text {stab }}(S) \rightarrow \pi_{*}(\mathrm{tmf}) \\
& \text { (Vergleich mit topologischen Modulformen). }
\end{aligned}
$$





\section{Inhaltsverzeichnis}

1 Hintergrund 3

1.1 Die Steenrod-Algebra . . . . . . . . . . . . . . . . . . . 3

1.1.1 Hopf-Algebren allgemein . . . . . . . . . . . . . . 3

1.1.2 Steenrod-Algebra . . . . . . . . . . . . . 5

1.1 .3 Milnor-Basis . . . . . . . . . . . . . . . . 6

1.1.4 Hopf-Unteralgebren . . . . . . . . . . . . . . . . . . . . 9

1.2 Die Adams-Spektralreihe . . . . . . . . . . . . . . . . . . . . . . . 9

1.2.1 Steenrod-Operationen auf der Kohomologie . . . . . . . . . 10

1.2.2 Kohomologie von Eilenberg-MacLane-Räumen . . . . . . . . . 12

1.2.3 Stabile Homotopietheorie . . . . . . . . . . . . . . . . . 13

1.2.4 Adams-Spektralreihe . . . . . . . . . . . . . . . 16

1.2 .5 Beispiele ...................... 19

1.3 Minimale Auflösungen . . . . . . . . . . . . . . . . . . . 20

1.3.1 Allgemein .................... . . 20 20

1.3.2 Der Standardalgorithmus . . . . . . . . . . . . . 22

2 Ein Lemma $\quad 27$

2.1 Das Lemma . . . . . . . . . . . . . . . . . . . . 27

2.1.1 Freundliche Sequenzen . . . . . . . . . . . . . . . 27

2.1 .2 Das Lemma . . . . . . . . . . . . . . . . . . . . 29

2.2 Weisen seiner Anwendung . . . . . . . . . . . . . . . . 32

2.2.1 Von Unten, zum Aufwärmen . . . . . . . . . . . . . . . 33

2.2 .2 Zulässige Unteralgebren . . . . . . . . . . . . . . 35

2.2.3 Von Unten, allgemein . . . . . . . . . . . . . . . . . . 43

2.2.4 Von Oben .................... . . . 45

2.2.5 Von Oben (de-Luxe-Variante) . . . . . . . . . . . 46

2.3 Der Algorithmus . . . . . . . . . . . . . . . . . . . . . . . . . . 48

2.3.1 Die Signaturfiltrierung . . . . . . . . . . . . . . . . 48

2.3 .2 Die Implementierung . . . . . . . . . . . . . . . . . . . . 51

2.3.3 Zur Wahl des passenden $B \ldots \ldots . . . . . . . . .53$

2.4 Resultate, Geschichte und Statistiken . . . . . . . . . . . . 55

3 Weitere Anwendungen $\quad 61$

$3.1 \operatorname{Ext}_{A}(M, N)$ für kleine $M$ und $N \ldots \ldots \ldots$. . . . . . . . 61

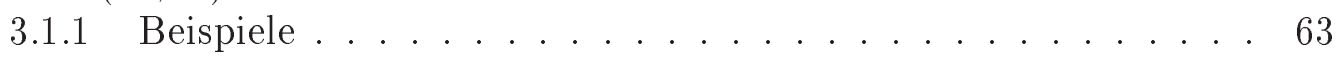

3.2 Wurzelinvarianten . . . . . . . . . . . . . . . . 72

3.3 Die Frobeniusoperation . . . . . . . . . . . . . . . . . . . . . . . 74

3.4 Hurewicz-Abbildung für tmf . . . . . . . . . . . . . . . . . . . 78

Danksagung 89 
Inhaltsverzeichnis 


\section{Hintergrund}

In diesem Kapitel geben wir zunächst eine rein algebraische Einführung in die Steenrod-Algebra, bevor wir dann im darauf folgenden Abschnitt die topologische Herkunft und Relevanz kurz diskutieren. Sodann widmen wir uns der Kohomologie der Steenrod-Algebra und diskutieren minimale Auflösungen und das bis dato gebräuchliche Verfahren ihrer Berechnung. All dies ist seit langem bekannt, und wird nur um der Geschlossenheit Willen rekapituliert. Ausführlicheres findet man zum Beispiel in [29], [39] und [32].

\subsection{Die Steenrod-Algebra}

\subsubsection{Hopf-Algebren allgemein}

Sei $k$ ein fest gewählter Grundkörper.

Definition 1.1.1. Eine unitale $k$-Algebra ist ein Tripel $(A, \mu, 1)$ bestehend aus einem $k$-Vektorraum $A$, einer assoziativen Multiplikation $\mu: A \otimes A \rightarrow A$ und einem Einselement $1: k \rightarrow A$.

Alle Tensorprodukte werden dabei über $k$ gebildet. Die Bedingung der Assoziativität und Unitalität bedeuten, daß die folgenden Diagramme kommutieren sollen:
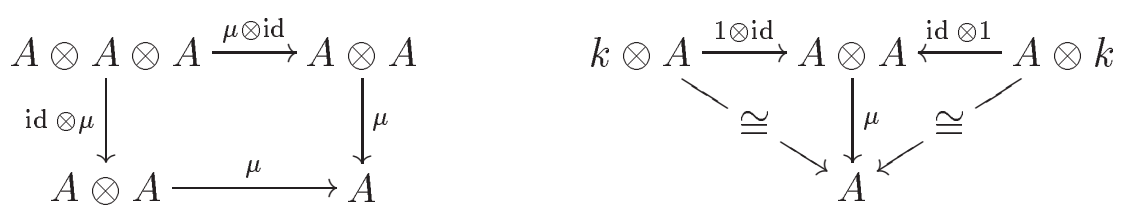

Durch Dualisieren erhält man den Begriff der augmentierten Koalgebra:

Definition 1.1.2. Eine augmentierte $k$-Koalgebra ist ein Tripel $(A, \Delta, \varepsilon)$ bestehend aus einem $k$-Vektorraum $A$, einer koassoziativen Komultiplikation $\Delta: A \rightarrow A \otimes A$ und einer Augmentation $\varepsilon: A \rightarrow k$.

Hier wird also die Kommutativität folgender Diagramme gefordert:
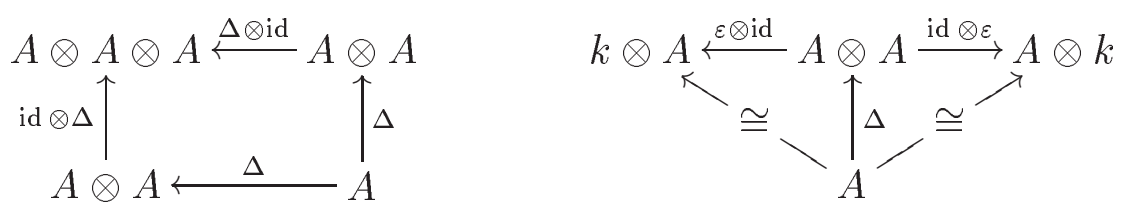

Kombiniert man beide Definitionen, so erhält man den Begriff der Hopf-Algebra: 
Definition 1.1.3. Eine Hopf-Algebra über $k$ ist ein Tupel $(A, \mu, \Delta, 1, \varepsilon)$, sodaß $(A, \mu, 1)$ eine unitale Algebra ist, $(A, \Delta, \varepsilon)$ eine augmentierte Koalgebra, und beide Strukturen kompatibel sind:

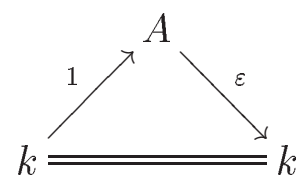

soll kommutieren. Außerdem soll $\Delta$ multiplikativ, und $\mu$ komultiplikativ sein.

Die letzten beiden Kompatibilitätsbedingungen sind äquivalent. Beide besagen gerade, daß das Diagramm

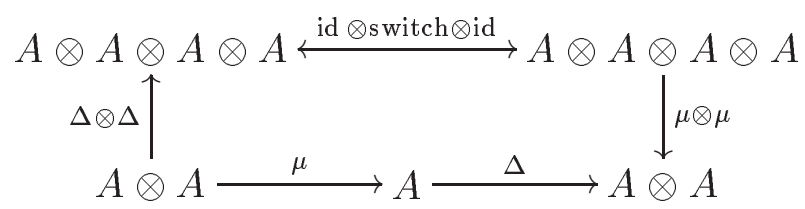

kommutiert. Falls $A$ graduiert ist, so muß beim Vertauschen die übliche Vorzeichenregel beachtet werden. Dazu setzt man $\operatorname{switch}(x \otimes y)=(-1)^{|x| \cdot|y|} y \otimes x$.

Für Moduln $M, N$ über einer Hopf-Algebra $A$ hat man ein internes Tensorprodukt $M \wedge N$. Als Vektorraum sei $M \wedge N=M \otimes_{k} N$. Die $A$-Operation sei diagonal, dh. durch

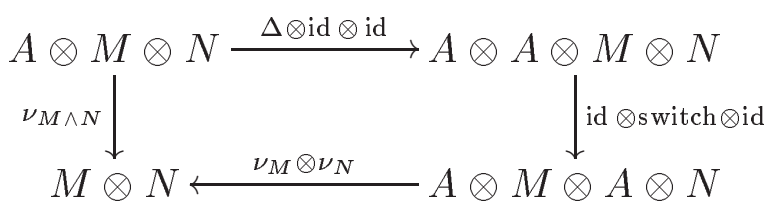

definiert. In Kurzschreibweise lautet dies $a(m \wedge n)=\sum(-1)^{|m| \cdot\left|a^{\prime \prime}\right|}\left(a^{\prime} m\right) \wedge\left(a^{\prime \prime} n\right)$. Dabei haben wir uns der gebräuchlichen symbolischen Notation $\Delta(a)=: \sum a^{\prime} \otimes a^{\prime \prime}$ bedient.

Definition 1.1.4. Eine Antipodenabbildung (oder Konjugation) auf einer Hopf-Algebra ist ein antimultiplikatives $\chi: A \rightarrow A$, sodaß $1 \circ \varepsilon=\mu($ id $\otimes \chi) \Delta$ gilt:

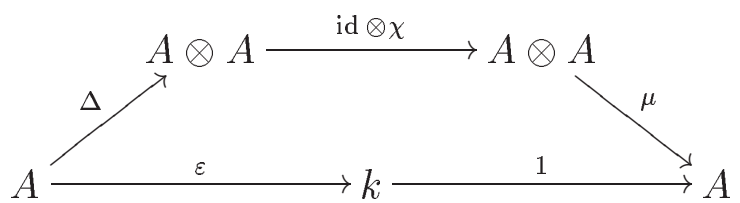

Zum Beispiel hat jede zusammenhängende, graduierte Hopf-Algebra automatisch eine solche Konjugation ([29], ch. 12.1), und falls $A$ kokommutativ ist, so ist diese involutiv: $\chi^{2}=$ id.

Für einen $k$-Vektorraum $M$ wird $A \otimes M$ durch Operation auf dem linken Faktor zu einem freien $A$-Modul.

Lemma 1.1.5. Hat A eine Antipodenabbildung, so ist die Abbildung

$$
A \otimes M \ni a \otimes m \mapsto \sum a^{\prime} \wedge a^{\prime \prime} m \in A \wedge M
$$

für alle A-Moduln $M$ ein A-linearer Isomorphismus. 
Insbesondere ist also $A \wedge M$ immer ein freier $A$-Modul.

Beweis. Man rechnet nach, daß

$$
A \wedge M \ni a \wedge m \mapsto \sum a^{\prime} \otimes \chi\left(a^{\prime \prime}\right) m \in A \otimes M
$$

ein Inverses ist. (Siehe zB. Seite 62.)

Es gibt zwei wichtige Beispielklassen, die man kennen sollte. Zum einen kann man jeder Gruppe $G$ den Gruppenring $k G$ zuordnen. Die Multiplikation auf $k G$ soll gerade von der Multiplikation $G \times G \rightarrow G$ induziert werden. Die Komultiplikation wird durch $\Delta(g)=g \otimes g$ für $g \in G$ erklärt. Die Antipodenabbildung wirkt durch $\chi(g)=g^{-1}$. Offenbar wird $k G$ dadurch zu einer kokommutativen Hopf-Algebra mit Konjugation.

Zum anderen kann man jeder kommutativen Hopf-Algebra mit Konjugation ein Gruppenschema zuordnen. Ist nämlich $A$ bzgl. $\mu$ ein kommutativer Ring, so kann man das zugehörige affine Schema $G=\operatorname{Spec} A$ bilden. Damit ist dann $G \times G=$ Spec $A \otimes A$. Da $\Delta: A \rightarrow A \otimes A, \chi: A \rightarrow A$ und $\varepsilon: A \rightarrow k$ multiplikativ sind, darf man sie als Abbildungen $G \times G \rightarrow G, G \rightarrow G$ bzw. Speck $=\{$ pt. $\} \rightarrow G$ betrachten. Obige Axiome besagen dann gerade, daß $G$ mit diesen Abbildungen als Multiplikation, Inversenbildung bzw. Inklusion der Eins, zu einem Gruppenobjekt in der Kategorie der $k$-Schemata wird. Diese Beziehung kann man auch umkehren: eine kommutative Hopf-Algebra mit Antipodenabbildung ist schlicht dasselbe wie ein affines Gruppenschema.

Bekanntlich ist der Dualraum einer Algebra eine Koalgebra, und umgekehrt. Man erhält also zu jeder Hopf-Algebra $(A, \mu, \Delta, 1, \varepsilon)$ eine duale Hopf-Algebra $\left(A^{*}, \mu^{\prime}, \Delta^{\prime}\right.$, $\left.1^{\prime}, \varepsilon^{\prime}\right)$ mit $\mu^{\prime}=\Delta^{*}, \Delta^{\prime}=\mu^{*}, 1^{\prime}=\varepsilon^{*}, \varepsilon^{\prime}=1^{*}$. Der Stern bezeichnet hier die kontragradiente Abbildung.

Ist $A$ endlich-dimensional (oder graduiert und lokal endlich-dimensional) so ist in natürlicher Weise $A=\left(A^{*}\right)^{*}$.

\subsubsection{Steenrod-Algebra}

Die Steenrod-Algebra, um die es im folgenden gehen wird, ist kokommutativ, nicht aber kommutativ. Man definiert sie am elegantesten als Dualraum ihres kommutativen Duals:

Definition 1.1.6. Sei $p$ eine Primzahl. Die duale, reduzierte Steenrod-Algebra zu $p$ ist folgende kommutative $\mathbb{F}_{p}$-Hopf-Algebra $A_{*}^{\text {red }}$ : Es sei $A_{*}^{\text {red }}=\mathbb{F}_{p}\left[\xi_{1}, \xi_{2}, \ldots\right]$ mit dem Koprodukt

$$
\Delta\left(\xi_{n}\right)=\sum_{i=0}^{n} \xi_{n-i}^{p^{i}} \otimes \xi_{i},
$$

wobei formal $\xi_{0}=1$ gesetzt werde. Außerdem sei $\varepsilon\left(\xi_{j}\right)=0$.

Das Koprodukt hat eine hübsche Interpretation durch die Algebra der additiven Potenzreihen. Darunter versteht man Potenzreihen der Form

$$
f_{\xi}(x)=x+\xi_{1} x^{p}+\xi_{2} x^{p^{2}}+\xi_{3} x^{p^{3}}+\cdots .
$$




\section{Hintergrund}

Für die Verknüpfung solcher Potenzreihen gilt nämlich über $\mathbb{F}_{p}$

$$
f_{\xi^{\prime}}\left(f_{\xi^{\prime \prime}}(x)\right)=\sum_{i \geq 0} \xi_{i}^{\prime}\left(\sum_{j \geq 0} \xi_{j}^{\prime \prime} x^{p^{j}}\right)^{p^{i}}=\sum_{i, j \geq 0} \xi_{i}^{\prime} \xi_{j}^{\prime \prime p^{i}} x^{p^{i+j}}=\sum_{n \geq 0} \xi_{n} x^{p^{n}}
$$

mit $\xi_{n}=\sum_{i+j=n} \xi_{j}^{\prime \prime p^{i}} \xi_{i}^{\prime}$. Das Koprodukt auf $A_{*}^{\text {red }}$ beschreibt also genau die Zusammensetzung additiver Potenzreihen. Die Konjugation auf $A_{*}^{\text {red }}$ wird üblicherweise mit $c: A_{*}^{\text {red }} \rightarrow A_{*}^{\text {red }}$ bezeichnet. Man setzt $\zeta_{n}=c\left(\xi_{n}\right)$. Die Beziehung zwischen den $\xi_{n}$ und den $\zeta_{n}$ kann man dann kurz und knapp durch $f_{\xi}^{-1}(x)=f_{\zeta}(x)$ beschreiben. $f_{\xi}^{-1}(x)$ ist dabei das Kompositionsinverse von $f_{\xi}(x)$.

$A_{*}^{\text {red }}$ wird als graduierte Hopf-Algebra betrachtet:

Definition 1.1.7. Für $p>2$ sei $\left|\xi_{i}\right|=2\left(p^{i}-1\right)$. Für $p=2$ sei $\left|\xi_{i}\right|=2^{i}-1$.

Die volle duale Steenrod-Algebra enthält für ungerades $p$ noch einen äußeren Anteil:

Definition 1.1.8. Die duale Steenrod-Algebra $A_{*}$ sei folgendermaßen definiert: Für $p=2$ sei $A_{*}=A_{*}^{\text {red }}$. Für $p>2$ sei $A_{*}=A_{*}^{\text {red }} \otimes E\left(\tau_{0}, \tau_{1}, \ldots\right)$, wobei $E(\cdots)$ die äußere Algebra über den angegebenen Erzeugenden bezeichne. Das Koprodukt sei auf den $\xi_{n}$ wie oben definiert. Für die $\tau_{n}$ setze man

$$
\Delta\left(\tau_{n}\right)=\tau_{n} \otimes 1+\sum_{0 \leq i \leq n} \xi_{n-i}^{p^{i}} \otimes \tau_{i} .
$$

Es sei $\left|\tau_{n}\right|=2 p^{n}-1$ und $\varepsilon\left(\tau_{j}\right)=0$.

Für den äußeren Anteil in $A_{*}$ ist unseres Wissens noch keine schöne Interpretation vorgeschlagen worden. Wir vermuten zwar, daß sich eine solche im Umkreis der Drinfeld-Moduln-Theorie finden ließe, wollen das hier aber nicht versuchen.

Jetzt können wir schließlich sagen, was die Steenrod-Algebra ist.

Definition 1.1.9. Die Steenrod-Algebra $A$ sei die zu $A_{*}$ duale Hopf-Algebra.

Das Dual der reduzierten dualen Steenrod-Algebra nennt man auch manchmal die reduzierte Steenrod-Algebra: $A^{\text {red }}=\left(A_{*}^{\text {red }}\right)^{*} . A^{\text {red }}$ ist eine Hopf-Unteralgebra von A.

\subsubsection{Milnor-Basis}

Da es im folgenden um das Rechnen in und mit der Steenrod-Algebra geht, ist uns die Definition $A=\left(A_{*}\right)^{*}$ nicht explizit genug. Milnor hat in [34] eine sehr bequeme Vektorraumbasis von $A$ angegeben und die Multiplikation in dieser Basis beschrieben. (Siehe auch [29], ch. 15.1). Dies wollen wir hier rekapitulieren.

Wir schreiben $\langle-,-\rangle: A \otimes A_{*} \rightarrow \mathbb{F}_{p}$ für die Dualitätspaarung zwischen $A$ und $A_{*}$. Eine Vektorraumbasis von $A_{*}^{\text {red }}$ wird durch die Menge der Monome $\xi^{R}=\xi_{1}^{r_{1}} \cdots \xi_{n}^{r_{n}}$ gebildet. Hierbei sei $R=\left(r_{1}, \ldots, r_{n}\right)$ (mit variablem $n$ ) eine Exponentenfolge. Wir bezeichnen die duale Basis von $A^{\text {red }}$ mit $P(R)$. Es gilt also

$$
P(R) \in A, \quad|P(R)|=\left|\xi^{R}\right|, \quad\left\langle P(R), \xi^{S}\right\rangle=\delta_{R, S}
$$


Definition 1.1.10. $\{P(R) \mid R$ Exponentenfolge $\}$ heißt Milnor-Basis von $A^{\text {red }}$.

Man überlegt sich leicht, daß das Koprodukt auf den $P(R)$ durch $\Delta(P(R))=$ $\sum_{E+F=R} P(E) \otimes P(F)$ gegeben ist. Für $p=2$ schreibt man üblicherweise $\operatorname{Sq}(R)$ statt $P(R)$. Ist in $R$ nur der erste Eintrag von Null verschieden, so schreibt man häufig $P^{k}$ statt $P(k)$ und $\mathrm{Sq}^{k}$ statt $\mathrm{Sq}(k)$.

Milnors Beschreibung der Multiplikation in $A^{\text {red }}$ lautet jetzt wie folgt:

Lemma 1.1.11. Seien $P(R)=P\left(r_{1}, \ldots, r_{n}\right)$ und $P(S)=P\left(s_{1}, \ldots, s_{n}\right)$ Elemente der Milnor-Basis. Dann ist

$$
P(R) \cdot P(S)=\sum_{X} \beta(X) P\left(t_{1}(X), \ldots, t_{n}(X)\right)
$$

wobei über Matrizen $X=\left(x_{i, j}\right)_{i, j \geq 0, i+j \neq 0}$ summiert wird, die folgenden Einschränkungen unterworfen sind:

- Die Summe der k-ten Spalte soll $s_{k}$ sein:

$$
s_{k}=x_{0, k}+x_{1, k}+x_{2, k}+\cdots+x_{n, k} .
$$

- Die gewichtete Summe der k-ten Zeile soll $r_{k}$ sein:

$$
r_{k}=x_{k, 0}+p \cdot x_{k, 1}+p^{2} \cdot x_{k, 2}+\cdots+p^{n} \cdot x_{k, n} .
$$

Der Koeffizient $\beta(X)$ ist das Produkt der diagonal gebildeten Multinomialkoeffizienten:

$$
\beta(X)=\prod_{k \geq 1}\left(x_{k, 0}\left|x_{k-1,1}\right| \cdots \mid x_{0, k}\right) \quad \text { mit } \quad\left(n_{1}|\cdots| n_{k}\right)=\frac{\left(n_{1}+\cdots+n_{k}\right) !}{n_{1} ! \cdots n_{k} !} .
$$

Die $t_{k}$ schließlich sind die diagonal gebildeten Summen

$$
t_{k}(X)=x_{k, 0}+x_{k-1,1}+\cdots+x_{0, k} .
$$

Zur Berechnung der hierbei auftretenden Multinomialkoeffizienten ist folgendes Lemma nützlich, dessen Beweis wir dem Leser als Übung empfehlen.

Lemma 1.1.12. Seien $m, n \in \mathbb{N}$ und prim.

1) Schreibt man $m=\sum m_{i} p^{i}, n=\sum n_{i} p^{i}$, mit $0 \leq m_{i}, n_{i}<p$, so ist $(m \mid n) \equiv$ $\prod_{i}\left(m_{i} \mid n_{i}\right) \bmod p$.

2) Für $m, n<p$ gilt

$$
\begin{array}{ll}
(m \mid n) \equiv 0 \bmod p & \text { falls } m+n \geq p \\
(m \mid n) \not \equiv 0 \bmod p & \text { falls } m+n<p .
\end{array}
$$

3) Es ist $\left(m_{1}|\cdots| m_{k}\right)=\left(m_{1} \mid m_{2}\right) \cdot\left(m_{1}+m_{2}\left|m_{3}\right| \cdots \mid m_{k}\right)$.

Kombiniert man die Aussagen dieses Lemmas, so sieht man 


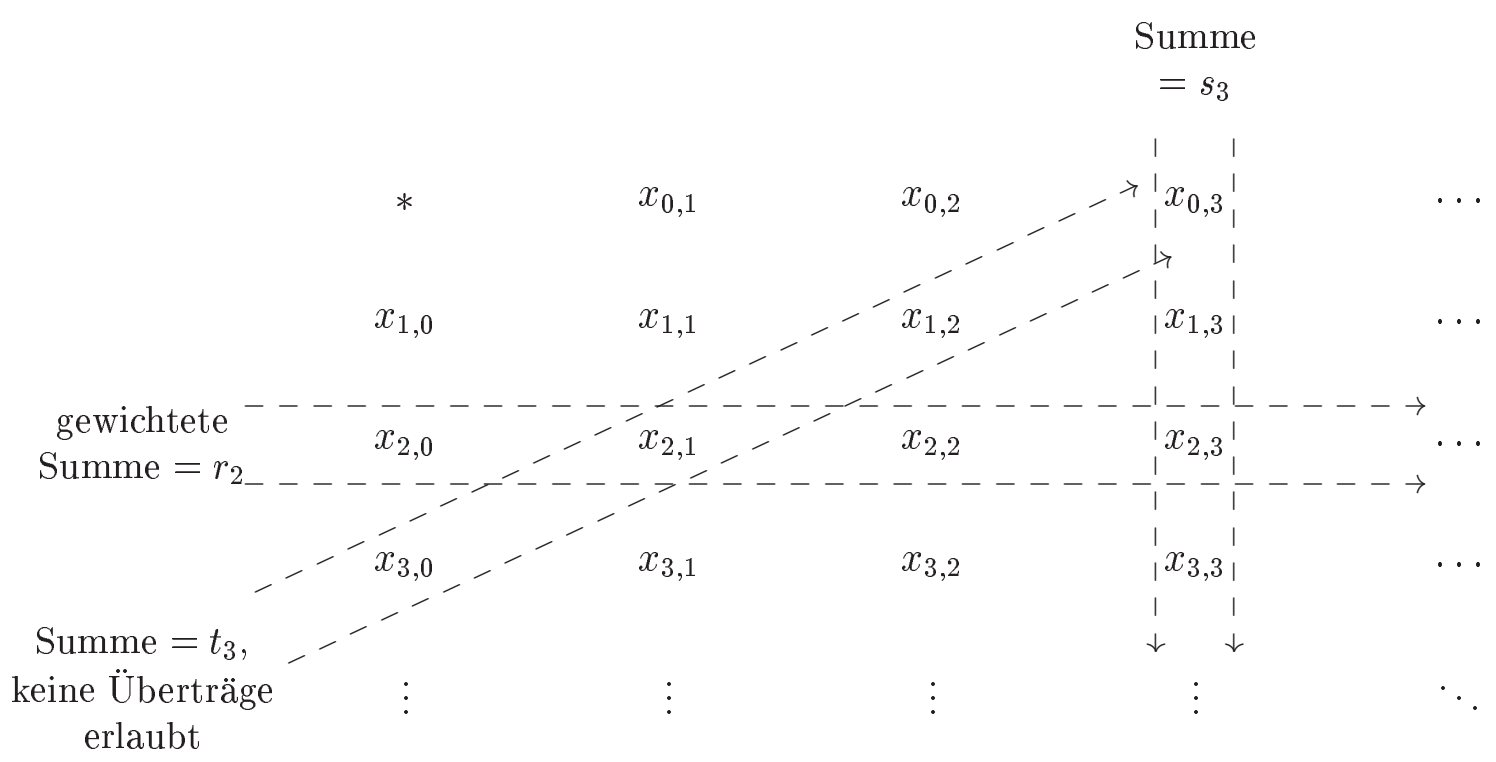

Abbildung 1.1: Multiplizieren in der Milnorbasis

Korollar 1.1.13. $\left(m_{1}|\cdots| m_{k}\right)$ ist genau dann $\not \equiv 0 \bmod p$ wenn bei der Addition $m_{1}+m_{2}+\cdots+m_{k}$ zur Basis $p$ keine Überträge entstehen.

Der Multiplikationsalgorithmus wird in Abbildung 1.1 bildlich dargestellt. Wir geben trotzdem noch ein Beispiel: Für $p=2$ erhält man $\mathrm{Sq}(4) \cdot \mathrm{Sq}(3)=\mathrm{Sq}(7)+0+$ $\mathrm{Sq}(1,2)$. Die zugehörigen Matrizen sehen so aus:

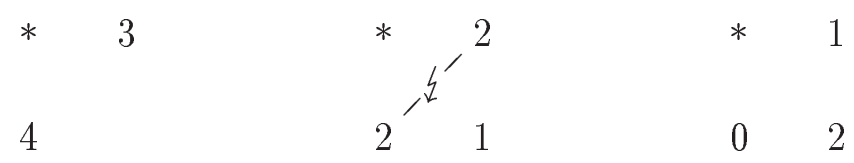

\ markiert dabei einen verbotenen Übertrag, der diesen Summanden annulliert.

Für ungerades $p$ muß man noch den äußeren Teil von $A_{*}$ mitbedenken. Sei dazu $Q_{i} \in A$ durch

$$
\left\langle Q_{i}, \tau^{\varepsilon} \xi^{R}\right\rangle= \begin{cases}1 & \tau^{\varepsilon} \xi^{R}=\tau_{i} \\ 0 & \text { sonst }\end{cases}
$$

definiert. Hier steht $\varepsilon=\left(\varepsilon_{0}, \varepsilon_{1}, \ldots\right)$ für eine nur aus Nullen und Einsen gebildete Exponentenfolge. Wir setzen $Q(\varepsilon)=Q^{\varepsilon}=\prod Q_{i}^{\varepsilon_{i}}$. Damit gilt

Lemma 1.1.14. Sei $p$ ungerade.

1) Die $Q_{i}$ erzeugen eine äußere Algebra $E\left(Q_{0}, Q_{1}, \ldots\right) \subset A$.

2) Die $Q_{i}$ vertauschen mit den $P(R)$ gemäß

$$
\left[P\left(r_{1}, \ldots, r_{n}\right), Q_{s}\right]=\sum_{t>0} Q_{s+t} P\left(r_{1}, \ldots, r_{t-1}, r_{t}-p^{s}, r_{t+1}, \ldots, r_{n}\right) .
$$

Falls hier $r_{t}-p^{s}<0$ ist, wird der entsprechende Summand weggelassen. 
3) $\{Q(\varepsilon) P(R) \mid R$ und $\varepsilon$ wie oben $\}$ ist die $z u\left\{\tau^{\varepsilon} \xi^{R}\right\} \subset A_{*}$ duale Vektorraumbasis von $A$.

Für $p=2$ setzt man üblicherweise $Q_{0}=\operatorname{Sq}^{1}, Q_{1}=\operatorname{Sq}(0,1), Q_{2}=\operatorname{Sq}(0,0,1)$, usw. Auch hier erzeugen die $Q_{i}$ eine äußere Unteralgebra. Die $Q_{i}$ werden oft als Milnorsche Bocksteinoperatoren bezeichnet.

Für alle $p$ gilt für das Koprodukt $\Delta\left(Q_{i}\right)=Q_{i} \otimes 1+1 \otimes Q_{i}$.

\subsubsection{Hopf-Unteralgebren}

Definition 1.1.15. Eine Unteralgebra $B \subset A$, die unter dem Koprodukt abgeschlossen ist, heißt Hopf-Unteralgebra von $A$.

Abgeschlossenheit bedeutet hier, daß $\Delta(B) \subset B \otimes B$ ist. Ein solches $B$ ist also selbst eine Hopf-Algebra.

Die Hopf-Unteralgebren der Steenrod-Algebra sind von Adams und Margolis in [3] vollständig klassifiziert worden. Dazu betrachtet man Funktionen

$$
\pi:\{1,2, \ldots\} \rightarrow\{0,1,2, \ldots, \infty\}, \quad \sigma:\{0,1,2, \ldots\} \rightarrow\{1,2\}
$$

und die folgenden Bedingungen

Für alle $i, j \geq 1$ gilt jeweils $\pi(i) \leq j+\pi(i+j)$ oder $\pi(j) \leq \pi(i+j)$.

Für alle $i \geq 1, j \geq 0$ mit $\sigma(i+j)=1$ gilt $\pi(i) \leq j$ oder $\sigma(j)=1$.

Für $p>2$ heißt das Paar $(\pi, \sigma)$ Profilfunktion, falls es 1.1 und 1.2 erfüllt; für $p=2$ betrachtet man $\pi$ alleine und verlangt nur 1.1.

$\mathrm{Zu}$ einer Profilfunktion assoziiert man die folgenden Unterräume von $A$ :

$$
\begin{aligned}
p>2: & B(\pi, \sigma) & =\left\{Q(\varepsilon) P(R) \mid r_{i}<p^{\pi(i)}, \varepsilon_{j}<\sigma(j)\right\} \\
p=2: & B(\pi) & =\left\{\operatorname{Sq}(R) \mid r_{i}<2^{\pi(i)}\right\}
\end{aligned}
$$

Damit gilt:

Satz 1.1.16 ([3]). Die $B(\pi, \sigma)$ (bzw. $B(\pi)$ für $p=2$ ) sind Hopf-Unteralgebren von $A$ und jede Hopf-Unteralgebra von $A$ ist von dieser Form.

Auf die $B(\pi, \sigma)$ werden wir in Abschnitt 2.2.2 zurückkommen.

\subsection{Die Adams-Spektralreihe}

Die Steenrod-Algebra wurde ursprünglich nicht, wie bei uns gerade geschehen, rein algebraisch definiert: man hat sie nicht er- sondern gefunden, und zwar bei der Untersuchung des Kohomologiefunktors $H^{*}\left(-; \mathbb{F}_{p}\right)$. Dem wenden wir uns nun zu. Klassische Texte zu diesem Thema sind [42] und [37]. 


\subsubsection{Steenrod-Operationen auf der Kohomologie}

Definition 1.2.1. Eine Kohomologieoperation ist eine natürliche Transformation $\theta: H^{i}\left(-; \mathbb{F}_{p}\right) \rightarrow H^{j}\left(-; \mathbb{F}_{p}\right)$.

Zur Klarstellung sei gesagt, daß wir hier die $H^{*}\left(-; \mathbb{F}_{p}\right)$ als Funktoren

$$
\operatorname{Top}_{*} \rightarrow \text { Set }
$$

betrachten. Wir beschränken uns also auf Räume, die einen ausgezeichneten Basispunkt besitzen, und verstehen demgemäß unter $H^{*}\left(X ; \mathbb{F}_{p}\right)$ immer die reduzierten Kohomologiegruppen. Da wir uns außerdem nicht mit Problemen und Pathologien aus der mengentheoretischen Topologie herumschlagen wollen, nehmen wir immer stillschweigend an, daß alle Räume zu CW-Komplexen homotopieäquivalent sind.

Die Definition besagt also, daß uns für jeden Raum $X \in$ Top $_{*}$ eine Abbildung $\theta_{X}: H^{i}\left(X ; \mathbb{F}_{p}\right) \rightarrow H^{j}\left(X ; \mathbb{F}_{p}\right)$ gegeben sein soll, die kein Homomorphismus zu sein braucht. Diese Abbildung soll für alle Räume auf die gleiche Weise definiert sein: vergleicht man nämlich zwei Räume $X, Y \in \mathbf{T o p}_{*}$ durch eine stetige Abbildung $f: X \rightarrow Y$, so soll das Diagramm

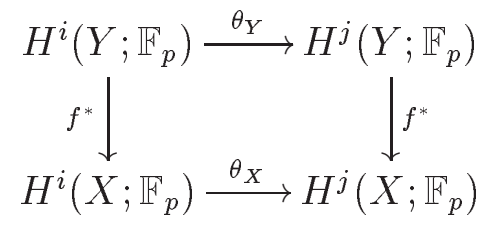

kommutieren.

Ein sehr einfaches Beispiel ist die Transformation $x \mapsto x^{k}$ von $H^{i}\left(-; \mathbb{F}_{p}\right)$ nach $H^{k i}\left(-; \mathbb{F}_{p}\right)$. Etwas anspruchsvoller ist die sogenannte Bocksteinoperation. Zu der kurzen exakten Sequenz

$$
0 \longrightarrow \mathbb{F}_{p} \stackrel{\cdot p}{\longrightarrow} \mathbb{Z} / p^{2} \mathbb{Z} \stackrel{\bmod p}{\longrightarrow} \mathbb{F}_{p} \longrightarrow 0
$$

gehört bekanntlich eine lange exakte Sequenz der Kohomologiegruppen

$$
\cdots \longrightarrow H^{i}\left(X ; \mathbb{Z} / p^{2} \mathbb{Z}\right) \stackrel{\bmod p}{\longrightarrow} H^{i}\left(X ; \mathbb{F}_{p}\right) \stackrel{\beta_{p}}{\longrightarrow} H^{i+1}\left(X ; \mathbb{F}_{p}\right) \longrightarrow \cdots
$$

$\beta_{p}$ ist besagte Bocksteinoperation und das einfachste Beispiel für die folgende

Definition 1.2.2. Eine stabile Kohomologieoperation der Dimension $n$ ist eine Familie $\theta=\left(\theta_{k}\right)_{k \in \mathbb{Z}}$ von Kohomologieoperationen

$$
H^{k}\left(X ; \mathbb{F}_{p}\right) \stackrel{\theta_{k}}{\longrightarrow} H^{n+k}\left(X ; \mathbb{F}_{p}\right),
$$

für die

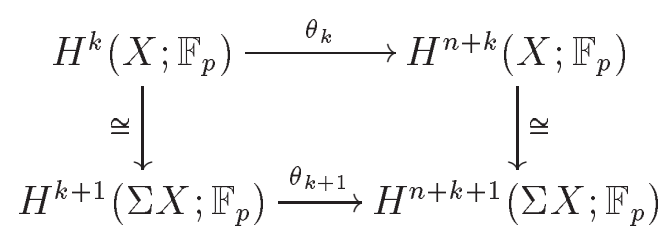

kommutiert. 
Hier ist $\Sigma X=C^{+} X \cup_{X} C^{-} X$ die Einhängung von $X$, die bekanntlich aus zwei Kegeln $C^{ \pm} X$ über $X$ durch Verklebung längs des gemeinsamen Bodens entsteht. Die im Diagramm auftretenden Einhängungsisomorphismen $H^{i}\left(X ; \mathbb{F}_{p}\right) \rightarrow H^{i+1}\left(\Sigma X ; \mathbb{F}_{p}\right)$ stammen aus der Mayer-Vietoris-Sequenz

$$
H^{i}\left(C^{+} X\right) \oplus H^{i}\left(C^{-} X\right) \rightarrow H^{i}(X) \stackrel{\cong}{\longrightarrow} H^{i+1}(\Sigma X) \rightarrow H^{i+1}\left(C^{+} X\right) \oplus H^{i+1}\left(C^{-} X\right)
$$

Da die Kegel zusammenziehbar sind, ist nämlich $H^{*}\left(C^{ \pm} X\right)=0$.

Die Bedeutung der Steenrod-Algebra für die Topologie wird nun durch folgenden Satz offenbar, zu dem nicht wenige Topologen beigetragen haben.

Satz 1.2.3. Die Steenrod-Algebra ist die Algebra der stabilen Kohomologieoperationen auf $H^{*}\left(-; \mathbb{F}_{p}\right)$.

Etwas ausführlicher gesagt:

- Für jedes $X$ ist $H^{*}\left(X ; \mathbb{F}_{p}\right)$ in natürlicher Weise ein Modul über der SteenrodAlgebra zu dieser Primzahl.

Insbesondere gehört also zu jedem $a \in A$ eine Kohomologieoperation

$$
H^{i}\left(X ; \mathbb{F}_{p}\right) \ni x \stackrel{L_{a}}{\longmapsto} a \cdot x \in H^{i+|a|}\left(X ; \mathbb{F}_{p}\right) .
$$

Damit gilt:

- Diese Kohomologieoperationen sind stabil.

- Jede stabile Kohomologieoperation ist von dieser Form für ein eindeutig bestimmtes $a \in A$.

Beispielsweise ist $Q_{0}$ gerade die oben beschriebene Bocksteinoperation.

Man beachte, daß insbesondere jede stabile Kohomologieoperation additiv ist. Außerdem kann keine nichttriviale stabile Kohomologieoperation die Dimension vermindern; dies gilt sogar ohne die Forderung der Stabilität.

Satz 1.2.4. Die A-Operation auf $H^{*}\left(X ; \mathbb{F}_{p}\right)$ hat folgende Eigenschaften: Für ungerades $p$ und $x, y \in H^{*}\left(X ; \mathbb{F}_{p}\right)$ gilt:

$$
\begin{aligned}
& \text { Cartan-Formel: } \quad P(R)(x y)=\sum_{E+F=R} P(E) x \cdot P(F) y . \\
& \text { Instabilität: } \quad|x|=2 r \quad \Rightarrow \quad P^{r} x=x^{p} \text {, } \\
& |x|<2 r \quad \Rightarrow \quad P^{r} x=0 . \\
& \text { Außerdem ist } \quad Q_{0} x=\beta_{p} x .
\end{aligned}
$$

Für $p=2$ hat man analog:

$$
\begin{aligned}
& \text { Cartan-Formel: } \quad \operatorname{Sq}(R)(x y)=\sum_{E+F=R} \operatorname{Sq}(E) x \cdot \operatorname{Sq}(F) y . \\
& \text { Instabilität: } \quad|x|=r \quad \Rightarrow \quad \mathrm{Sq}^{r} x=x^{2} \text {, } \\
& |x|<r \quad \Rightarrow \quad \operatorname{Sq}^{r} x=0 \text {. } \\
& \text { Außerdem ist } \quad Q_{0} x=\beta_{2} x \text {. }
\end{aligned}
$$

Die Beziehung $\mathrm{Sq}^{|x|} x=x^{2}$ ist für die Bezeichnung Sq verantwortlich: Sq steht für "square" und wird auch so gelesen. 


\subsubsection{Kohomologie von Eilenberg-MacLane-Räumen}

Sei $R$ eine abelsche Gruppe, $n \in \mathbb{N}$. Zur Erinnerung:

Definition 1.2.5. Ein Raum $X \in$ Top $_{*}$ heißt Eilenberg-MacLane-Raum vom Typ $(R, n)$ falls

$$
\pi_{j}(X)= \begin{cases}R & \text { für } j=n \\ 0 & \text { sonst. }\end{cases}
$$

Ein solches $X$ existiert zu jedem $R$ und $n$ und ist bis auf Homotopieäquivalenz eindeutig bestimmt. Es wird üblicherweise durch $K(R, n)$ bezeichnet.

Wir betrachten den Fall $R=\mathbb{F}_{p}$. Aus dem Satz von Hurewicz folgt, daß

$$
H^{k}\left(K\left(\mathbb{F}_{p}, n\right) ; \mathbb{F}_{p}\right)= \begin{cases}0 & \text { für } k<n, \\ \mathbb{F}_{p} & \text { für } k=n .\end{cases}
$$

Sei $\iota_{n} \in H^{n}\left(K\left(\mathbb{F}_{p}, n\right) ; \mathbb{F}_{p}\right)$ die damit der $1 \in \mathbb{F}_{p}$ entsprechende Fundamentalklasse. Der folgende Satz zeigt nochmals die Bedeutung der Steenrod-Algebra für die Homotopietheorie auf:

Satz 1.2.6. $H^{*}\left(K\left(\mathbb{F}_{p}, n\right), \mathbb{F}_{p}\right)$ ist die von $\iota_{n}$ frei erzeugte instabile Algebra über der Steenrod-Algebra.

Unter einer instabilen Algebra ist dabei ein graduierter $A$-Modul $M$ mit einer graduiert-kommutativen Multiplikation $M \otimes M \rightarrow M$ zu verstehen, für den die oben als "Cartan-Formel" bzw. "Instabilität" bezeichneten Rechenregeln gelten.

Damit sind Homologie und Kohomologie der Eilenberg-MacLane-Räume explizit bekannt. Stellt man Eilenberg-MacLane-Räume und Sphären in dieser Beziehung gegenüber, so ergibt sich das folgende Bild, in dem eine gewisse Spiegelsymmetrie zu erkennen ist.

\begin{tabular}{c|c|c} 
& Homologie & Homotopie \\
\hline$K\left(\mathbb{F}_{p}, n\right)$ & Steenrod-Algebra & einfach \\
\hline$S^{n}$ & einfach & sehr kompliziert
\end{tabular}

Wir werden weiter unten bei der Diskussion der Adams-Spektralreihe hierauf zurückkommen.

Sei $A \tilde{\iota}_{n}$ der freie $A$-Modul über einem Erzeugenden $\tilde{\iota}_{n}$ der Dimension $n$. Wir betrachten die $A$-lineare Abbildung $\phi_{n}: A \tilde{\iota}_{n} \rightarrow H^{*}\left(K\left(\mathbb{F}_{p}, n\right), \mathbb{F}_{p}\right)$ mit $\tilde{\iota}_{n} \mapsto \iota_{n}$.

Lemma 1.2.7. $\phi_{n}$ ist in den Dimensionen $<2 n$ ein Isomorphismus.

Beweisskizze. Sei $X=K\left(\mathbb{F}_{p}, n\right)$. Die Instabilitätsrelationen in $H^{*}\left(X ; \mathbb{F}_{p}\right)$ setzen erst in der Dimension $p n$ mit $P^{n / 2} \iota_{n}=\iota_{n}^{p}$ ein; da sich daraus keine Relationen zwischen den $a \iota_{n}$ mit $|a|<n$ herleiten lassen, ist $\phi_{n}$ bis zur Dimension $p n-1$ injektiv.

Andererseits ist $\phi_{n}$ bis zur Dimension $2 n-1$ auch surjektiv: Da $H^{*}\left(X ; \mathbb{F}_{p}\right)$ durch Steenrod-Operationen und Multiplikationen aus $\iota_{n}$ erzeugt wird, muß sich jede Klasse, die nicht im Bild von $\phi_{n}$ ist, aus Produkten zusammensetzen. Da die Kohomologie von $X$ aber erst in der Dimension $n$ anfängt, hat jedes nichttriviale Produkt mindestens Dimension $2 n$. 
Geht man nun geschickt zum Limes über die $K\left(\mathbb{F}_{p}, n\right)$ über, so kann man aus den $H^{*}\left(K\left(\mathbb{F}_{p}, n\right) ; \mathbb{F}_{p}\right)$ einen freien $A$-Modul herausschälen:

Korollar 1.2.8. Der Limes

$$
H \mathbb{F}_{p}^{k} H \mathbb{F}_{p}:=\lim _{\leftarrow} H^{k+n}\left(K\left(\mathbb{F}_{p}, n\right) ; \mathbb{F}_{p}\right)
$$

ist ein freier A-Modul mit einem Erzeugenden ८ in der Dimension Null.

Um die Bildung solcher Limiten und ihre homotopietheoretische Interpretation geht es im nächsten Abschnitt.

\subsubsection{Stabile Homotopietheorie}

Stabile Homotopietheorie ist eine Approximation an die Homotopietheorie, die neben vielen anderen Vorzügen auch den nicht unbedeutenden hat, einfacher als die gewöhnliche zu sein. Es ist unmöglich, auf wenigen Seiten eine auch nur annähernd adäquate Einführung in dieses wichtige und umfangreiche Gebiet zu geben. Wir beschränken uns deshalb auf die "bare essentials". Der wißbegierige Leser möge sich aus [43], [2] oder [29] weitere Hinweise besorgen. Wer aktuellere Entwicklungen verstehen möchte, kommt um die technisch anspruchsvolleren [22] und [18] nicht herum.

Definition 1.2.9. Seien $X, Y \in \operatorname{Top}_{*}, X$ kompakt. Die Elemente des Kolimes

$$
\{X, Y\}:=\underset{\rightarrow}{\operatorname{colim}}\left[\Sigma^{k} X, \Sigma^{k} Y\right]
$$

heißen stabile Homotopieklassen.

Nach Konstruktion hat man eine natürliche Abbildung $[X, Y] \rightarrow\{X, Y\}$, und eine kanonische Bijektion $\{X, Y\} \cong\{\Sigma X, \Sigma Y\}$. Aus letzterer folgt, daß $\left\{\Sigma^{k} X, \Sigma^{l} Y\right\}$ für beliebige ganze Zahlen $k, l \in \mathbb{Z}$ Sinn macht: dazu setze man einfach

$$
\left\{\Sigma^{k} X, \Sigma^{l} Y\right\}:=\left\{\Sigma^{n+k} X, \Sigma^{n+l} Y\right\} \quad \text { sobald } n+k \geq 0 \text { und } n+l \geq 0 .
$$

Man erhält somit eine Kategorie, wenn man als Objekte "formale Einhängungen" $\Sigma^{k} X, k \in \mathbb{Z}$, und als Morphismen die stabilen Homotopiemengen wählt. Diese Kategorie $\mathbf{S W}$ ist als Spanier-Whitehead-Kategorie bekannt. Man hat offenbar einen kanonischen Funktor $\mathbf{T o p}_{*} \rightarrow \mathbf{S W}$ mit $X \mapsto \Sigma^{0} X$.

Beim Vergleich zwischen stabilen und gewöhnlichen Homotopiemengen zeigt sich, daß die $\{X, Y\}$ immer noch geometrisch interpretiert werden können:

Satz 1.2.10 (Freudenthal's Einhängungssatz). Für $\operatorname{dim} X \leq 2 \cdot \operatorname{conn} Y$ ist $[X, Y] \rightarrow[\Sigma X, \Sigma Y]$ bijektiv.

Hier ist conn $Y$ die Konnektivität von $Y: 1+\operatorname{conn} Y=\min \left\{j \mid \pi_{j}(Y) \neq 0\right\}$. Da man $\operatorname{dim} \Sigma^{k} X=k+\operatorname{dim} X$ und conn $\Sigma^{k} Y=k+\operatorname{conn} Y$ hat, wird der Kolimes in der Definition von $\{X, Y\}$ angenommen: es ist $\{X, Y\}=\left[\Sigma^{k} X, \Sigma^{k} Y\right]$ für alle hinreichend großen $k$. 


\begin{tabular}{c|ccccccccccc}
$n \backslash k$ & 0 & 1 & 2 & 3 & 4 & 5 & 6 & 7 & 8 & 9 & 10 \\
\hline 1 & $\infty$ & 0 & 0 & 0 & 0 & 0 & 0 & 0 & 0 & 0 & 0 \\
2 & $\infty$ & $\infty$ & 2 & 2 & 12 & 2 & 2 & 3 & 15 & 2 & $2^{2}$ \\
3 & $\infty$ & 2 & 2 & 12 & 2 & 2 & 3 & 15 & 2 & $2^{2}$ & 12.2 \\
4 & $\infty$ & 2 & 2 & $\infty .12$ & $2^{2}$ & $2^{2}$ & 24.3 & 15 & 2 & $2^{3}$ & 120.12 .2 \\
5 & $\infty$ & 2 & 2 & 24 & 2 & 2 & 2 & 30 & 2 & $2^{3}$ & 72.2 \\
6 & $\infty$ & 2 & 2 & 24 & 0 & $\infty$ & 2 & 60 & 24.2 & $2^{3}$ & 72.2 \\
7 & $\infty$ & 2 & 2 & 24 & 0 & 0 & 2 & 120 & $2^{3}$ & $2^{4}$ & 24.2 \\
8 & $\infty$ & 2 & 2 & 24 & 0 & 0 & 2 & $\infty .120$ & $2^{4}$ & $2^{5}$ & $24^{2} .2$ \\
9 & $\infty$ & 2 & 2 & 24 & 0 & 0 & 2 & 240 & $2^{3}$ & $2^{4}$ & 24.2 \\
10 & $\infty$ & 2 & 2 & 24 & 0 & 0 & 2 & 240 & $2^{2}$ & $\infty .2^{3}$ & 12.2 \\
11 & $\infty$ & 2 & 2 & 24 & 0 & 0 & 2 & 240 & $2^{2}$ & $2^{3}$ & 6.2 \\
12 & $\infty$ & 2 & 2 & 24 & 0 & 0 & 2 & 240 & $2^{2}$ & $2^{3}$ & 6 \\
$\vdots$ & $\vdots$ & $\vdots$ & $\vdots$ & $\vdots$ & $\vdots$ & $\vdots$ & $\vdots$ & $\vdots$ & $\vdots$ & $\vdots$ & $\vdots$
\end{tabular}

Abbildung 1.2: $\pi_{n+k}\left(S^{n}\right)$ nach [46], zitiert nach [39]. a.b.c . steht für $\mathbb{Z}_{a} \oplus \mathbb{Z}_{b} \oplus \cdots$, $\infty$ steht für $\mathbb{Z}, 0$ für $0 . \mathbb{Z}_{a}$ steht für $\mathbb{Z} / a \mathbb{Z}$.

Definition 1.2.11. $\pi_{n}^{\text {stab }}(X):=\left\{S^{n}, X\right\}$ heißt $n$-te stabile Homotopiegruppe von $X$.

Da $S^{k}=\Sigma^{k} S^{0}$ ist, hat $\operatorname{man} \pi_{n+k}^{\text {stab }}\left(S^{k}\right)=\pi_{n}^{\text {stab }}\left(S^{0}\right)$ für alle $n, k$. Man schreibt deswegen auch einfach $\pi_{n}^{\text {stab }}(S)$ oder gar $\pi_{n}^{\text {stab }}$ für diese Gruppe. $\pi_{n}^{\text {stab }}(S)$ wird $n$ Stamm oder auch n-te stabile Homotopiegruppe der Sphäre genannt. Abbildung 1.2 zeigt einen Teil der von Toda in [46] berechneten Homotopiegruppen $\pi_{n+k}\left(S^{n}\right)$. Dort kann man die Stabilisierung dieser Gruppen sehr schön erkennen.

In der stabilen Homotopietheorie erweitert man die Definition von $\{X, Y\}$ so, daß man für $X$ und $Y$ weit mehr als nur kompakte Räume einsetzen darf.

Definition 1.2.12. Ein Präspektrum ist eine Folge von Räumen $\left(E_{n}\right)_{n \geq 0}$ aus Top mit Strukturabbildungen $\sigma_{n}: \Sigma E_{n} \rightarrow E_{n+1}$.

Ein Beispiel hierfür haben wir schon gesehen: die Eilenberg-MacLane-Räume $K(R, n)$ lassen sich durch Abbildungen

$$
\Sigma K(R, n) \rightarrow K(R, n+1)
$$

miteinander verbinden, und genau über diese Abbildungen haben wir oben den Limes $\lim H^{n+k}\left(K\left(\mathbb{F}_{p}, n\right) ; \mathbb{F}_{p}\right)$ gebildet. Ein anderes Beispiel sind die Einhängungspräspektren zu $X \in \operatorname{Top}_{*}$ : hier setzt man $E_{n}=\Sigma^{n} X$, die Strukturabbildungen $\sigma_{n}: \Sigma \Sigma^{n} X \rightarrow \Sigma^{n+1} X$ seien die Identität.

Generell sollte man sich ein Präspektrum $E$ als gerichtetes System

$$
E_{0} \stackrel{\Sigma^{-1} \sigma_{0}}{\longrightarrow} \Sigma^{-1} E_{1} \stackrel{\Sigma^{-2} \sigma_{1}}{\longrightarrow} \Sigma^{-2} E_{2} \stackrel{\Sigma^{-3} \sigma_{2}}{\longrightarrow} \Sigma^{-3} E_{3} \stackrel{\Sigma^{-4} \sigma_{3}}{\longrightarrow} \cdots
$$

vorstellen, daß sich nur leider in $\mathbf{T o p}_{*}$ nicht interpretieren läßt, geschweige, daß es dort einen Limes hätte. Die Homotopietheoretiker sind nun aber, über den langen 
und mühsamen Weg von [23] durch [2] zu [18], in die glückliche Lage gekommen, diese fehlenden Limiten adjungieren zu können: man hat eine Kategorie Spectra konstruiert, in der ebenfalls Homotopietheorie betrieben werden kann. Die Objekte dieser Kategorie heißen Spektren. Nach Konstruktion hat man einen Funktor

$$
\Sigma^{\infty}: \operatorname{Top}_{*} \rightarrow \text { Spectra }
$$

der jedem $X$ das zugehörige Einhängungsspektrum zuordnet. Dabei ist für kompaktes $X$

$$
\left[\Sigma^{\infty} X, \Sigma^{\infty} Y\right]_{\mathrm{Ho}(\text { Spectra })}=\{X, Y\}
$$

Der Übergang von Top zu $_{*}$ Spectra hat also gerade den oben beschriebenen Stabilisierungseffekt.

Spektren lassen sich nicht nur ein-, sondern auch aushängen. Hat man ein Präspektrum $E$ so kann man deshalb das zugehörige gerichtete System in Spectra realisieren

$$
\Sigma^{\infty} E_{0} \stackrel{\Sigma^{-1} \sigma_{0}}{\longrightarrow} \Sigma^{\infty-1} E_{1} \stackrel{\Sigma^{-2} \sigma_{1}}{\longrightarrow} \Sigma^{\infty-2} E_{2} \stackrel{\Sigma^{-3} \sigma_{2}}{\longrightarrow} \Sigma^{\infty-3} E_{3} \stackrel{\Sigma^{-4} \sigma_{3}}{\longrightarrow} \cdots
$$

und dort besitzt es einen Kolimes: das zu $E$ assoziierte Spektrum. Umgekehrt läßt sich auch jedes Spektrum als ein solcher Kolimes schreiben.

Stabile Homotopietheorie ist nun nichts anderes als die Homotopietheorie der Spektren. In vielerlei Hinsicht ist Homotopietheorie in Spectra anders als Homotopietheorie in Top $_{*}$. Wir stellen ein paar Eigenheiten kurz vor:

- Ho(Spectra) ist eine additive Kategorie: für $E, F \in \mathbf{S p e c t r a}$ ist $[E, F]$ eine abelsche Gruppe.

Dies folgt daraus, daß Spektren immer als Einhängung geschrieben werden können: $E=\Sigma^{2} \Sigma^{-2} E$ und schon in der gewöhnlichen Homotopietheorie ist $\left[\Sigma^{2} X, Y\right]$ immer eine abelsche Gruppe. Tatsächlich gilt sogar

- Ho(Spectra) ist eine triangulierte Kategorie in Bezug auf die Gesamtheit der Dreiecke

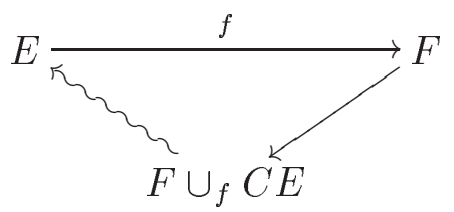

Dabei ist $f: E \rightarrow F$ eine beliebige Abbildung aus Spectra.

Ein Pfeil $X \leadsto Y$ bezeichnet dabei eine Abbildung $X \rightarrow \Sigma Y$. Die Aussage bedeutet im wesentlichen, daß $[Z,-]$ und $[-, Z]$ für jedes $Z \in$ Spectra dieses Dreieck in eine lange exakte Sequenz überführen. Insbesondere erhält man im Fall $Z=S^{n}$ zu jeder Inklusion $A \longmapsto X$ eine lange exakte Homotopiesequenz

$$
\cdots \rightarrow \pi_{n}(A) \rightarrow \pi_{n}(X) \rightarrow \pi_{n}(X / A) \rightarrow \pi_{n-1}(A) \rightarrow \cdots .
$$

$X \mapsto \pi_{*}(X)$ ist also für Spektren $X$ eine Homologietheorie. 
- Jede verallgemeinerte Kohomologietheorie $E^{*}(-): \mathbf{T o p}_{*} \rightarrow \mathbf{A b}$ setzt sich kanonisch auf Spectra fort und ist dort darstellbar.

Darstellbarkeit heißt, daß es ein im wesentlichen eindeutig bestimmtes Spektrum $E$ gibt, für das auf natürliche Weise $E^{k}(X)=\left[\Sigma^{k} X, E\right]$ für alle $X \in$ Spectra gilt.

Auf Spektren läßt sich der Smash-Produkt-Funktor $\wedge: \mathbf{T o p}_{*} \times \mathbf{T o p}_{*} \rightarrow \mathbf{T o p}_{*}$ übertragen.

- Jede verallgemeinerte Homologietheorie $E_{*}(-):$ Top $_{*} \rightarrow$ Ab setzt sich kanonisch auf Spectra fort und ist dort darstellbar.

Hier soll Darstellbarkeit heißen, daß es ein kanonisches $E \in$ Spectra mit $E_{k}(X)=$ $\pi_{k}(E \wedge X)$ für alle $X \in$ Spectra gibt.

Unser wichtigstes Beispiel ist die gewöhnliche Kohomologie: es ist

$$
H^{k}(X ; R)=\left[\Sigma^{k} X, H R\right], \quad H_{k}(X ; R)=\pi_{k}(H R \wedge X)
$$

wobei

$$
H R:=\underset{\rightarrow}{\operatorname{colim}} \Sigma^{\infty-n} K(R, n)
$$

das Eilenberg-MacLane-Spektrum zu $R \in \mathbf{A b}$ ist. Dieses könnte man auch durch

$$
\pi_{j}(H R)= \begin{cases}R & j=0 \\ 0 & \text { sonst }\end{cases}
$$

bis auf Homotopieäquivalenz charakterisieren.

In Korollar 1.2.8 haben wir bereits gesehen, daß $H \mathbb{F}_{p}{ }^{*} H \mathbb{F}_{p}$ ein freier $A$-Modul über $\iota \in H \mathbb{F}_{p}{ }^{0} H \mathbb{F}_{p}$ ist. Wie wir jetzt wissen, können wir dies auch als

$$
H \mathbb{F}_{p}{ }^{*} H \mathbb{F}_{p}=\left[\Sigma^{*} H \mathbb{F}_{p}, H \mathbb{F}_{p}\right]=\pi_{*}\left(\operatorname{End}\left(H \mathbb{F}_{p}\right)\right)
$$

schreiben. Da Endomorphismen einen Ring bilden, ist folgender Satz wohl nicht unplausibel:

Satz 1.2.13. $\pi_{*}\left(\operatorname{End}\left(H \mathbb{F}_{p}\right)\right)$ ist die Steenrod-Algebra zur Primzahl $p$.

Dem $\iota$ aus Korollar 1.2.8 entspricht einfach die identische Abbildung auf $H \mathbb{F}_{p}$.

\subsubsection{Adams-Spektralreihe}

Wir nähern uns dem Ende unseres kleinen Schnellkurses in Homotopietheorie. Als letztes möchten wir noch erklären, was die Adams-Spektralreihe ist und tut, bevor wir uns dann unserer eigentlichen Bestimmung, nämlich der unverfälschten, reinen Algebra, zuwenden werden.

Erinnern wir uns an die kleine Tabelle in Abschnitt 1.2.2, jetzt in stabilisierter Form:

\begin{tabular}{c|c|c} 
& Kohomologie & Homotopie \\
\hline$H \mathbb{F}_{p}$ & Steenrod-Algebra & einfach \\
\hline$S$ & einfach & sehr kompliziert
\end{tabular}


Die Idee hinter der Adams-Spektralreihe besteht darin, ein ziemlich beliebiges Spektrum $X$ so gut wie möglich aus Eilenberg-MacLane-Spektren nachzubauen. Bei diesem Prozess

- entsteht aus den bekannten und ziemlich banalen Homotopiegruppen der Eilenberg-MacLane-Spektren die womöglich sehr komplizierte Struktur der Homotopiegruppen $\pi_{*}(X)$

während gleichzeitig

- die Kohomologie von $X$ aus der Kohomologie der Eilenberg-MacLane-Spektren, dh. aus freien $A$-Moduln, nachgebaut wird.

Die Komplexität von $\pi_{*}(X)$ sollte also dem Maß der Unfreiheit von $H^{*}\left(X ; \mathbb{F}_{p}\right)$ als $A$-Modul entsprechen.

Die Idee des Nachbaus wird - allerdings in invertierter Form: als Abbau - in der folgenden Definition präzisiert. Sei $X$ ein Spektrum und $p$ prim.

Definition 1.2.14 (vgl. [39], Ch. 2.1). Eine Adams-Auflösung von $X$ bezüglich $H \mathbb{F}_{p}$ ist ein Diagramm

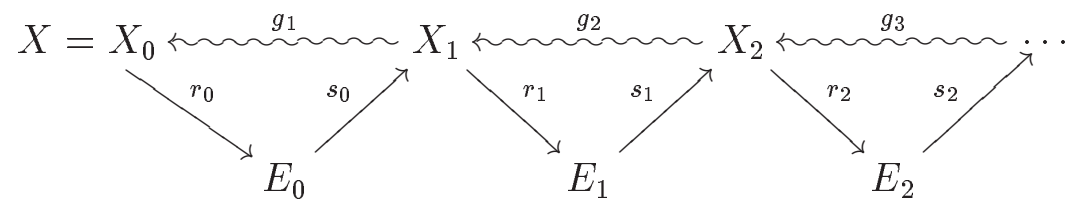

von Spektren $X_{i}$, sodaß folgendes gilt:

- Jedes $g_{i}$ induziert in der Kohomologie $H^{*}\left(-; \mathbb{F}_{p}\right)$ die Null.

- Jedes $E_{i}$ ist ein verallgemeinertes Eilenberg-MacLane-Spektrum $V$ zu einem graduierten $\mathbb{F}_{p}$-Vektorraum $V_{i}$.

Ist also $V_{i}=\oplus_{j} V_{i, j}$ die Zerlegung in homogene Bestandteile, so soll

$$
E_{i}=\bigvee_{j} \Sigma^{j} H V_{i, j}
$$

sein. Damit gilt insbesondere $\pi_{j}\left(H V_{i}\right)=V_{i, j}$.

- In jedem Dreieck ist $X_{i+1}=E_{i} \cup_{r_{i}} C X_{i}$.

Letzteres darf und sollte man sich als $\Sigma X_{i+1} \subset X_{i}$ mit $X_{i} / \Sigma X_{i+1}=E_{i}$ vorstellen; beim Schritt von $X_{i}$ zu $\Sigma X_{i+1}$ wird also nur ein verallgemeinertes EilenbergMacLane-Spektrum von $X_{i}$ weggenommen.

Man kann zeigen, daß jedes Spektrum eine solche Adams-Auflösung besitzt. Aus einer solchen Auflösung ergeben sich

- eine Filtrierung von $\pi_{*}(X)$ durch die Bilder $\pi_{*+k}\left(X_{k}\right) \rightarrow \pi_{*}(X)$, 
- eine Folge langer exakter Homotopiesequenzen

$$
\cdots \rightarrow \pi_{t+1}\left(X_{s+1}\right) \rightarrow \pi_{t}\left(X_{s}\right) \rightarrow \pi_{t}\left(E_{s}\right) \rightarrow \pi_{t}\left(X_{s+1}\right) \rightarrow \cdots
$$

und

- eine $A$-freie Auflösung

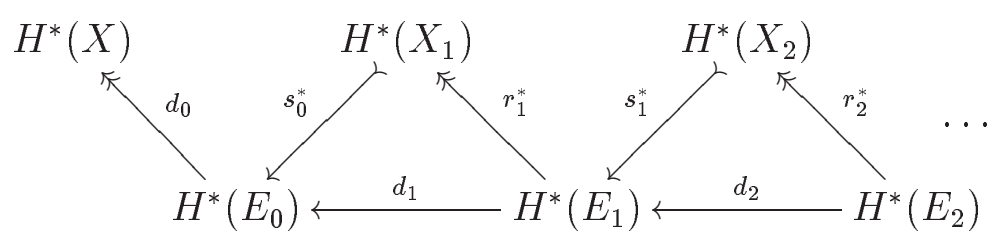

von $H^{*}\left(X ; \mathbb{F}_{p}\right)$.

Diese Zutaten werden im folgenden Satz systematisch zusammengebracht.

Satz 1.2.15. Zu jeder Adams-Auflösung von $X$ gehört eine Spektralreihe $E_{r}^{s, t}, d_{r}$ : $E_{r}^{s, t} \rightarrow E_{r}^{s+r, t-r-1}, r \geq 1$, mit:

- $E_{1}^{s, t}=\pi_{t}\left(E_{s}\right)=\operatorname{Hom}_{A}^{t}\left(H^{*}\left(E_{s}\right), \mathbb{F}_{p}\right)$.

- $E_{2}^{s, t}=\operatorname{Ext}_{A}^{s, t}\left(H^{*}(X), \mathbb{F}_{p}\right)$.

- Falls alle Homotopiegruppen $\pi_{k}(X)$ endlich erzeugt sind und $\pi_{k}(X)=0$ für $k \ll 0$ gilt, so konvergiert die Spektralreihe gegen die $p$-Lokalisierungen $\pi_{t-s}($ $X)_{(p)}$.

- Die $E_{r}$ und $d_{r}$ für $r \geq 2$ hängen nicht mehr von der Wahl der Adams-Auflösung $a b$.

Die zur Konvergenz nötigen Annahmen sind in den von uns anvisierten Fällen immer erfüllt. Auch ist klar, daß man Konvergenz nicht gegen die Homotopiegruppen selbst, sondern bestenfalls gegen die $p$-Lokalisierung dieser Gruppen erwarten darf; schließlich kann $H^{*}\left(-; \mathbb{F}_{p}\right)$ Torsionsphänomene zu den von $p$ verschiedenen Primzahlen einfach nicht sehen.

Die Bigraduierung der Ext-Gruppen erklärt sich daraus, daß die $H^{*}\left(E_{i} ; \mathbb{F}_{p}\right)$ selber schon eine Graduierung mitbringen. In dem Ausdruck $\operatorname{Ext}_{A}^{s, t}(-,-)$ steht $s$ für den üblichen homologischen Grad und $t$ für diesen internen Grad.

Wir erwähnen noch eine Verallgemeinerung:

Satz 1.2.16. Für Spektren $X$ und $Y$ läßt sich aus einer Adams-Auflösung von $Y$ eine Spektralreihe

$$
E_{2}^{s, t}=\operatorname{Ext}_{A}^{s, t}\left(H^{*}(Y), H^{*}(X)\right) \Rightarrow\left[\Sigma^{t-s} X, Y\right]_{(p)}
$$

konstruieren, die unter vergleichbaren Bedingungen konvergiert. 


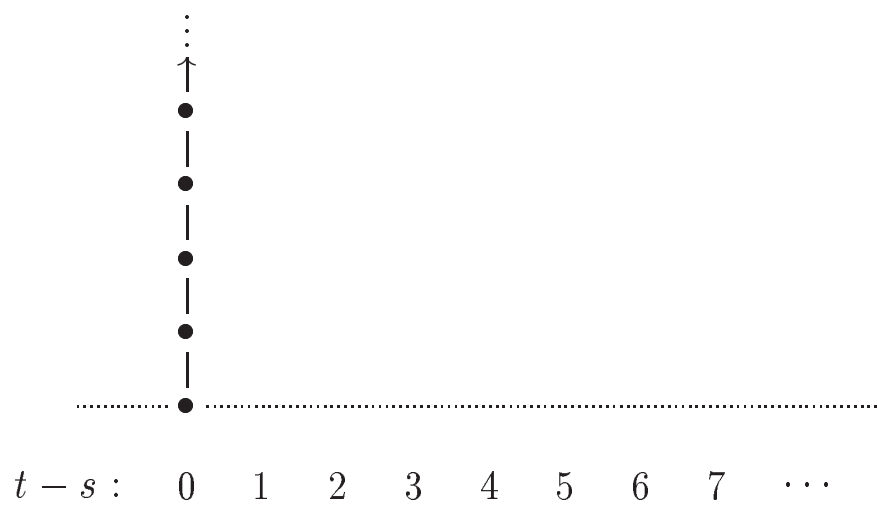

Abbildung 1.3: $E_{2}$-Term für $\pi_{*}(H \mathbb{Z})$

\subsubsection{Beispiele}

Wir geben zwei Beispiele zur Illustration der Spektralsequenz.

$X=H \mathbb{Z}$ : Unabhängig von $p$ sieht der $E_{2}$-Term wie in Abbildung 1.3 aus. Dabei haben wir das gebräuchliche Koordinatensystem für solche Darstellungen mit $(x, y)=(t-s, s)$ verwendet. Im Bild ist in jedem Kästchen eine Basis von

$$
\operatorname{Ext}_{A}^{s, t}\left(H^{*}\left(H \mathbb{Z} ; \mathbb{F}_{p}\right), \mathbb{F}_{p}\right)= \begin{cases}\mathbb{F}_{p} & t-s=0 \\ 0 & t-s \neq 0\end{cases}
$$

durch Punkte dargestellt. Die Spektralreihe kollabiert, weil keine nichttrivialen Differentiale möglich sind. Eine Adams-Auflösung von $H \mathbb{Z}$ wird durch

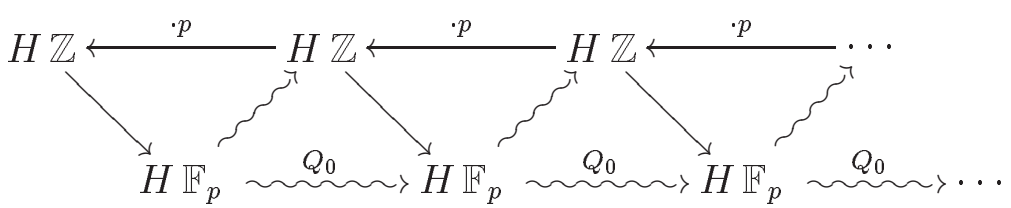

gegeben. Die zugehörige Adams-Filtrierung von $\pi_{*}(H \mathbb{Z})=\pi_{0}(H \mathbb{Z})=\mathbb{Z}$ ist gerade

$$
\mathbb{Z} \supset p \mathbb{Z} \supset p^{2} \mathbb{Z} \supset p^{3} \mathbb{Z} \supset \cdots
$$

und was man im Bild sieht sind die Quotienten

$$
E_{2}^{s, s}=E_{\infty}^{s, s}=p^{s} \mathbb{Z}_{(p)} / p^{s+1} \mathbb{Z}_{(p)}=p^{s} \mathbb{Z} / p^{s+1} \mathbb{Z} \cong \mathbb{F}_{p}
$$

Aus der Adams-Auflösung ergibt sich übrigens, daß $H^{*}\left(H \mathbb{Z} ; \mathbb{F}_{p}\right)=A / A Q_{0}$ ist.

$X=S$ : Wir betrachten hier nur $p=2$. Für die Sphäre sieht der $E_{2}$-Term schon komplizierter aus. Abbildung 1.4 zeigt den Anfangsbereich $t-s \leq 9$. Die vertikalen Linien zeigen die Wirkung $\operatorname{Ext}_{A}^{s, t} \rightarrow \operatorname{Ext}_{A}^{s+1, t+1}$ der Multiplikation $x \mapsto h_{0} x$. Dabei ist $h_{0} \in E_{2}^{1,1}$ das von Null verschiedene Element. Die diagonalen Linien zeigen ganz analog die Multiplikation mit $0 \neq h_{1} \in E_{2}^{1,2}$. Auch diese Spektralreihe kollabiert, allerdings nur in dem von uns betrachteten Bereich. Man erkennt, daß $\pi_{k}(S)_{(2)}$ für $k \leq 9$ eine Kompositionsreihe mit den folgenden Quotienten hat: 


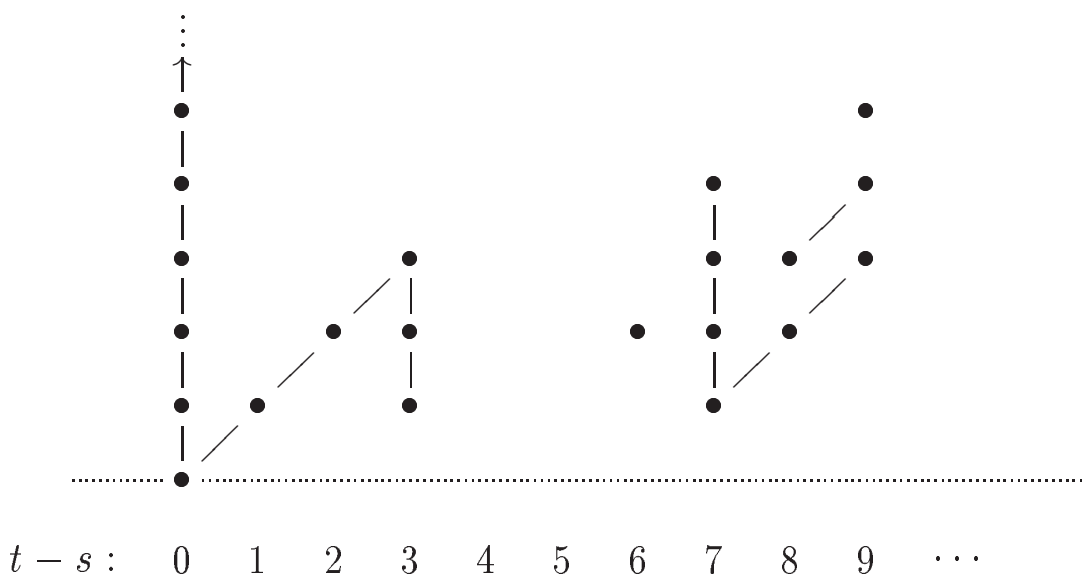

Abbildung 1.4: $E_{2}$-Term für $\pi_{*}(S)_{(2)}$ bis zur Dimension 9

$\begin{array}{lllll}\pi_{0}(S) & : \mathbb{F}_{2}, \mathbb{F}_{2}, \mathbb{F}_{2}, \mathbb{F}_{2}, \ldots & \pi_{5}(S) & : & 0 \\ \pi_{1}(S) & : \mathbb{F}_{2} & \pi_{6}(S) & : \mathbb{F}_{2} \\ \pi_{2}(S) & : \mathbb{F}_{2} & \pi_{7}(S) & : \mathbb{F}_{2}, \mathbb{F}_{2}, \mathbb{F}_{2}, \mathbb{F}_{2} \\ \pi_{3}(S) & : \mathbb{F}_{2}, \mathbb{F}_{2}, \mathbb{F}_{2} & \pi_{8}(S) & : \mathbb{F}_{2}, \mathbb{F}_{2} \\ \pi_{4}(S) & : 0 & \pi_{9}(S) & : \mathbb{F}_{2}, \mathbb{F}_{2}, \mathbb{F}_{2}\end{array}$

Man vergleiche dies mit Abbildung 1.2.

Mehr zur Adams-Spektralreihe findet der interessierte Leser in [39]. Wir wollen aber jetzt zum algebraischen Kern dieser Arbeit kommen, und beenden deswegen hier den topologischen Einschub.

\subsection{Minimale Auflösungen}

Wir haben hoffentlich den Leser davon überzeugt, daß die Ext-Gruppen $\operatorname{Ext}_{A}^{s, t}(M, N)$ in der Homotopietheorie eine wichtige Rolle spielen. Um Methoden zu ihrer Berechnung wird es im folgenden gehen.

\subsubsection{Allgemein}

Will man Kohomologiegruppen berechnen, so kommt man am Begriff der Auflösung nicht vorbei: es gibt kein algorithmisches Verfahren, das solche Gruppen direkt, dh. ohne Bezug auf eine Auflösung, bestimmen könnte.

$A$ sei im folgenden eine graduierte, zusammenhängende, lokal endlich-dimensionale Hopf-Algebra mit Konjugation über einem Körper $k$. Mit $I$ bezeichnen wir das Augmentationsideal $I=\operatorname{ker} \varepsilon: A \rightarrow k$. Alle von uns betrachteten Moduln seien von unten beschränkt, dh. es sei $M_{j}=0$ für $j \ll 0$. Sei $M$ ein $A$-Modul.

Definition 1.3.1. Eine Auflösung von $M$ über $A$ ist ein Kettenkomplex

$$
C_{0} \stackrel{d_{1}}{\longleftarrow} C_{1} \stackrel{d_{2}}{\longleftarrow} C_{2} \stackrel{d_{3}}{\longleftarrow} C_{3} \stackrel{d_{4}}{\longleftarrow} \ldots
$$


aus freien $A$-Moduln $C_{s}$ mit einer Augmentation $d_{0}: C_{0} \rightarrow M$, sodaß

$$
M \stackrel{d_{0}}{\longleftarrow} C_{0} \stackrel{d_{1}}{\longleftarrow} C_{1} \stackrel{d_{2}}{\longleftarrow} C_{2} \stackrel{d_{3}}{\longleftarrow} C_{3} \stackrel{d_{4}}{\longleftarrow} \cdots
$$

exakt ist.

Wir betrachten also ausschließlich freie Auflösungen, unter anderem weil über der Steenrod-Algebra sowieso jeder von unten beschränkte, projektive Modul frei ist ([29], Ch. 13.3, Th. 12). Ist $M=k$, so spricht man auch schlicht von einer Auflösung von $A$.

Hat man eine solche Auflösung, so setzt man bekanntlich

$$
\operatorname{Tor}_{s, t}^{A}(M, N)=H_{s, t}\left(N \otimes_{A} C_{*}\right) \quad \text { und } \quad \operatorname{Ext}_{A}^{s, t}(M, N)=H^{s, t}\left(\operatorname{Hom}_{A}\left(C_{*}, N\right)\right) .
$$

Tatsächlich reicht es, Auflösungen von $k$ zu betrachten, denn man hat $M \cong M \wedge k$ und das folgende

Lemma 1.3.2. Ist $C_{*}$ eine Auflösung von $M$, so ist $N \wedge C_{*}$ eine Auflösung von $N \wedge M$.

Beweis. $N \wedge C_{*}$ ist wieder exakt, weil wir das Tensorprodukt über einem Körper bilden, und aufgrund von Lemma 1.1.5 ist $N \wedge C_{*}$ wieder ein Komplex freier $A$ Moduln.

$$
\operatorname{Ext}_{A}^{s, t}(M, k) \text { und } \operatorname{Tor}_{s, t}^{A}(M, k) \text { sind zueinander duale Vektorräume, denn es gilt }
$$

Lemma 1.3.3. Für jeden $A$-Modul $M$ ist $\left(k \otimes_{A} M\right)^{*}=\operatorname{Hom}_{A}(M, k)$.

Beweis. Als $A$-Modul ist $k=A / I$, weil $I=\operatorname{ker} \varepsilon: A \rightarrow k$ und $\varepsilon$ surjektiv ist. Also ist $k \otimes_{A} M=M / I M$ für jeden $A$-Modul $M$. Ein $\psi: M \rightarrow k$ ist aber genau dann $A$-linear, wenn es auf $I M$ verschwindet, denn $I$ operiert auf dem Bild als Null. Also ist $\operatorname{Hom}_{A}(M, k)=\operatorname{Hom}_{k}(M / I M, k)=\operatorname{Hom}_{k}\left(k \otimes_{A} M, k\right)$ wie behauptet.

Im folgenden seien $C_{*}, C_{*}^{\prime}$, etc. Auflösungen von $k$ über $A$.

Definition 1.3.4. Eine Auflösung $C_{*}$ heißt minimal, wenn die folgenden, untereinander äquivalenten, Bedingungen erfüllt sind:

1) Das Differential auf $k \otimes_{A} C_{*}$ ist Null.

2) Das Differential auf $\operatorname{Hom}_{A}\left(C_{*}, k\right)$ ist Null.

3) Wählt man $A$-Basen in $C_{*}$, so stammt in der diesbezüglichen Matrixdarstellung des Differentials jeder Eintrag aus dem Augmentationsideal $I$.

Beweis der Äquivalenz. 1) $\Leftrightarrow 2)$ folgt aus Lemma 1.3.3, denn $k \otimes_{A} C_{*}$ und $\operatorname{Hom}_{A}\left(C_{*}\right.$, $k$ ) sind zueinander $k$-duale Kettenkomplexe. 3 ) ist nur eine Umformulierung von 1): das Differential auf $k \otimes_{A} C_{*}$ erhält man gerade aus der in 3) betrachteten Matrix durch Reduktion modulo $I$.

Wir werden im nächsten Abschnitt sehen, daß minimale Auflösungen existieren. 
Definition 1.3.5. Seien $C_{*}, C_{*}^{\prime}$ Auflösungen desselben Moduls $M$. Eine Kettenabbildung $\phi: C_{*} \rightarrow C_{*}^{\prime}$, die die Identität auf $M$ hebt, nennen wir Vergleichsabbildung.

Bekanntlich, lassen sich zwei beliebige Auflösungen immer durch solche Abbildungen vergleichen.

Lemma 1.3.6. Seien $C_{*}$ und $C_{*}^{\prime}$ Auflösungen von $k$ und $\phi: C_{*} \rightarrow C_{*}^{\prime}$ eine Vergleichsabbildung. Dann gilt:

1) $C_{*}$ minimal $\Rightarrow \phi$ ist injektiv.

2) $C_{*}^{\prime}$ minimal $\Rightarrow \phi$ ist surjektiv.

3) $C_{*}$ und $C_{*}^{\prime}$ minimal $\Rightarrow \phi$ ist ein Isomorphismus.

Beweis. Wir zeigen zunächst 3). Bekanntlich stiftet eine Vergleichsabbildung einen kanonischen Isomorphismus $H\left(k \otimes_{A} C_{*}\right) \cong H\left(k \otimes_{A} C_{*}^{\prime}\right)$, da beide Seiten gerade $\operatorname{Tor}_{*, *}^{A}(k, k)$ sind. Aufgrund der Minimalität ist aber $k \otimes_{A} C_{*}=H\left(k \otimes_{A} C_{*}\right)$ und $k \otimes_{A} C_{*}^{\prime}=H\left(k \otimes_{A} C_{*}^{\prime}\right)$. Also ist zumindest

$$
\text { id } \otimes \phi: k \otimes_{A} C_{*} \rightarrow k \otimes_{A} C_{*}^{\prime}
$$

ein Isomorphismus. Wir betrachten nun die exakten Sequenzen $I^{n+1} \longmapsto I^{n} \rightarrow$ $I^{n} / I^{n+1}$. Da $I^{n} / I^{n+1}$ als $A$-Modul eine Summe von Kopien von $k$ ist, ergibt sich durch Induktion, daß auch jedes

$$
\text { id } \otimes \phi: A / I^{n} \otimes_{A} C_{*} \rightarrow A / I^{n} \otimes_{A} C_{*}^{\prime}
$$

ein Isomorphismus ist. Da $A \rightarrow A / I^{n}$ in den Dimensionen $<n$ bijektiv ist, erhält man im Limes die Behauptung.

1) und 2) ergeben sich als Korollar. Wählt man nämlich eine beliebige Vergleichsabbildung $\psi: C_{*}^{\prime} \rightarrow C_{*}$ in die entgegengesetzte Richtung, so ist im Fall 1) $\psi \circ \phi$, und im Fall 2) $\phi \circ \psi$ ein Automorphismus.

Korollar 1.3.7. Je zwei minimale Auflösungen sind isomorph. Die minimale Auflösung ist ein Retrakt jeder anderen Auflösung.

Insbesondere ist die minimale Auflösung auch dadurch charakterisiert, daß sie die kleinstmögliche Auflösung ist.

\subsubsection{Der Standardalgorithmus}

Beim algorithmischen Berechnen von Auflösungen hat man es mit partiellen Auflösungen zu tun, und nicht mit vollständigen Auflösungen, die ja im allgemeinen unendlich-dimensionale Objekte sind.

Definition 1.3.8. Setze (siehe Abbildung 1.5)

$$
\begin{aligned}
& R_{s, t}=\left\{(u, v) \in \mathbb{Z}^{2} \mid u \leq s, v \leq t\right\} \\
& R_{s, t}^{o}=R_{s, t} \backslash\{(s, t)\} .
\end{aligned}
$$




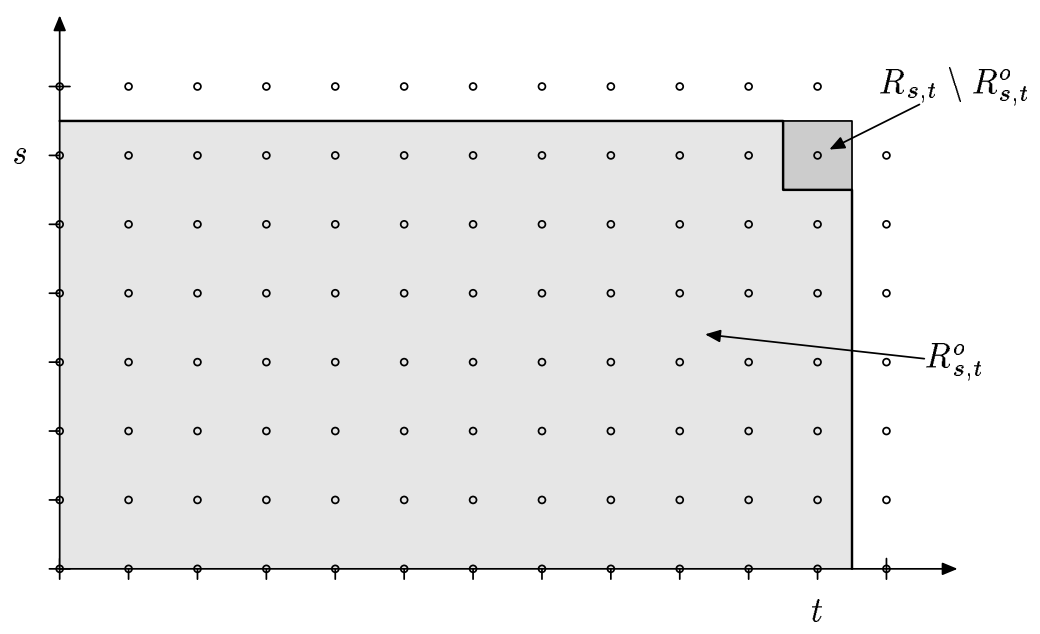

Abbildung 1.5: $R_{s, t}$ und $R_{s, t}^{o}$

Für $X=R_{s, t}$ oder $X=R_{s, t}^{o}$ nennen wir einen Kettenkomplex $\left(C_{*}, d_{*}\right)$ aus $A$-freien $C_{s}$ mit einer Augmentation $d_{0}: C_{0} \rightarrow k$, sodaß

$$
k \stackrel{d_{0}}{\longleftarrow} C_{0} \stackrel{d_{1}}{\longleftarrow} C_{1} \stackrel{d_{2}}{\longleftarrow} C_{2} \stackrel{d_{3}}{\longleftarrow} C_{3} \stackrel{d_{4}}{\longleftarrow} \ldots
$$

in allen Bigraden $(u, v) \in X$ exakt ist, eine $X$-Auflösung von $A$. Zur bequemeren Bezugnahme vereinbaren wir

$$
\begin{array}{ll}
R_{s, t} \text {-Auflösung }=: & \text { partielle Auflösung bis }(s, t), \\
R_{s, t}^{o} \text {-Auflösung }=: & \text { in }(s, t) \text { erweiterbare Auflösung. }
\end{array}
$$

Beispielsweise ist $C_{*}^{\text {anf }}$ mit $C_{0}^{\text {anf }}=A, d_{0}=\varepsilon$ und $C_{s}^{\text {anf }}=0$ für $s>0$ eine partielle Auflösung bis zu $(0,0)$. Dies heißt ja einfach, daß $\varepsilon: A \rightarrow k$ in der Dimension 0 bijektiv ist.

Wie der Name schon verspricht, kann man $\operatorname{Ext}_{A}^{s, t}(k, k)$ und $\operatorname{Tor}_{s, t}^{A}(k, k)$ aus einer partiellen Auflösung teilweise berechnen:

Lemma 1.3.9. Ist $C_{*}$ eine partielle Auflösung bis $z u(s, t)$, so ist

$$
\begin{aligned}
& \operatorname{Ext}_{A}^{u, v}(k, k)=H^{u, v}\left(\operatorname{Hom}_{A}\left(C_{*}, k\right)\right), \\
& \operatorname{Tor}_{u, v}^{A}(k, k)=H_{u, v}\left(k \otimes_{A} C_{*}\right)
\end{aligned}
$$

für $u<s$ und $v \leq t$.

Beweis. Klar. Siehe auch Korollar 3.1.2.

$C_{*}^{\text {anf }}$ dient als Ausgangspunkt für die Konstruktion einer minimalen Auflösung. Zur Ausdehnung folgt man dem

Erweiterungsschritt. Sei $C_{*}$ eine in $(s, t)$ erweiterbare Auflösung.

1) Berechne $H_{s, t}\left(C_{*}\right)$ und wähle eine $k$-Basis $c_{1}, \ldots, c_{n} \in H_{s, t}\left(C_{*}\right)$. 
2) Wähle Zykel $z_{1}, \ldots, z_{n} \in C_{s, t}$ mit $z_{i} \in c_{i}$.

3) Füge neue Erzeugende $g_{1}, \ldots, g_{n}$ mit $d_{s+1}\left(g_{i}\right)=z_{i}$ in $C_{s+1, t}$ ein:

$$
\begin{aligned}
C_{s+1}^{\text {neu }} & =C_{s+1} \oplus \Sigma^{t} A g_{1} \oplus \cdots \oplus \Sigma^{t} A g_{n}, \\
d_{s+1}^{\text {neu }}\left(g_{i}\right) & =z_{i} .
\end{aligned}
$$

Ist nun $C_{*}$ in $(s, t)$ erweiterbar, so ist $C_{*}^{\text {neu }}$ eine partielle Auflösung bis $(s, t)$. Die neuen Erzeugenden töten nach Konstruktion gerade die störende Homologiegruppe $H_{s, t}\left(C_{*}\right)$, und durch das Einfügen der neuen Erzeugenden hat man im Bereich $R_{s, t}^{o}$ nichts geändert, sodaß $C_{*}^{\text {neu }}$ dort exakt bleibt.

Zur Konstruktion einer partiellen minimalen Auflösung bis zu $(s, t)$ geht man nun so vor: man beginnt mit $C_{*}^{\text {anf }}$ und erweitert dies schrittweise gemäß folgendem Schema:

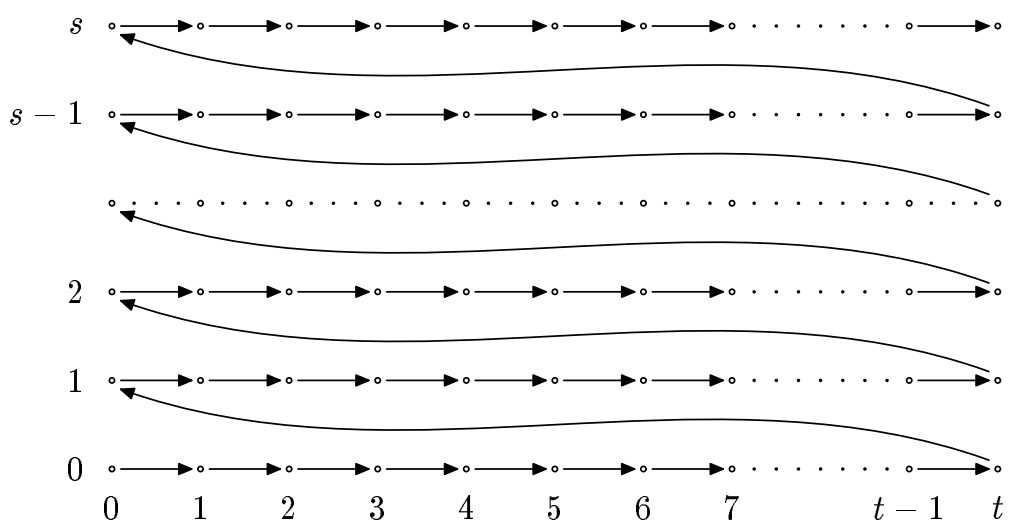

Daß dies funktioniert sagt

Lemma 1.3.10. Ist $C_{*}$ aus $C_{*}^{\text {anf }}$ durch eine Folge von Erweiterungsschritten entstanden, so ist es minimal, dh. das Differential auf $k \otimes_{A} C_{*}$ ist Null.

Beweis. Nach Annahme hat $C_{*}$ eine Filtrierung

$$
C_{*}=F^{s, t} C_{*} \supset F^{s, t-1} C_{*} \supset \cdots \supset F^{s, 0} C_{*} \supset F^{s-1, t} C_{*} \supset \cdots \supset F^{0, t} C_{*} \supset \cdots \supset F^{0,0} C_{*}=C_{*}^{\text {anf }}
$$

durch Unterkomplexe $F^{u, v} C_{*}$, die aus ihrem Vorgänger durch einen Erweiterungsschritt entstanden sind. Wir müssen zeigen, daß für jedes Erzeugende $g \in C_{u, v}$ das Differential $d(g)$ in $I C_{*}$ liegt. Schreibe dazu $d(g)=\sum_{i} a_{i} g_{i}$ mit $a_{i} \in A$ und $g_{i}$ Erzeugende aus $C_{u-1, *}$. Man erhält $0=d^{2}(g)=\sum_{i} a_{i} d\left(g_{i}\right)$. Wir betrachten die Homologieklasse der rechten Seite in $F^{u-1, v-1} C_{*}$. Falls $\left|a_{i}\right|>0$ ist $\left|g_{i}\right|<v$, sodaß $a_{i} g_{i} \in F^{u-1, v-1} C_{*}$; die Homologieklasse $\left[a_{i} d\left(g_{i}\right)\right] \in H_{u-1, v}\left(F^{u-1, v-1} C_{*}\right)$ ist also Null. In $H_{u-1, v}\left(F^{u-1, v-1} C_{*}\right)$ ist somit

$$
\sum_{i \text { mit } a_{i} \in k} a_{i}\left[d\left(g_{i}\right)\right]=0
$$

In den Bezeichnungen des Erweiterungsschritts ist dies eine $k$-lineare Relation zwischen den $c_{i}$, die aufgrund der linearen Unabhängigkeit null sein muß. Also sind alle $a_{i} \in I$, wie behauptet. 


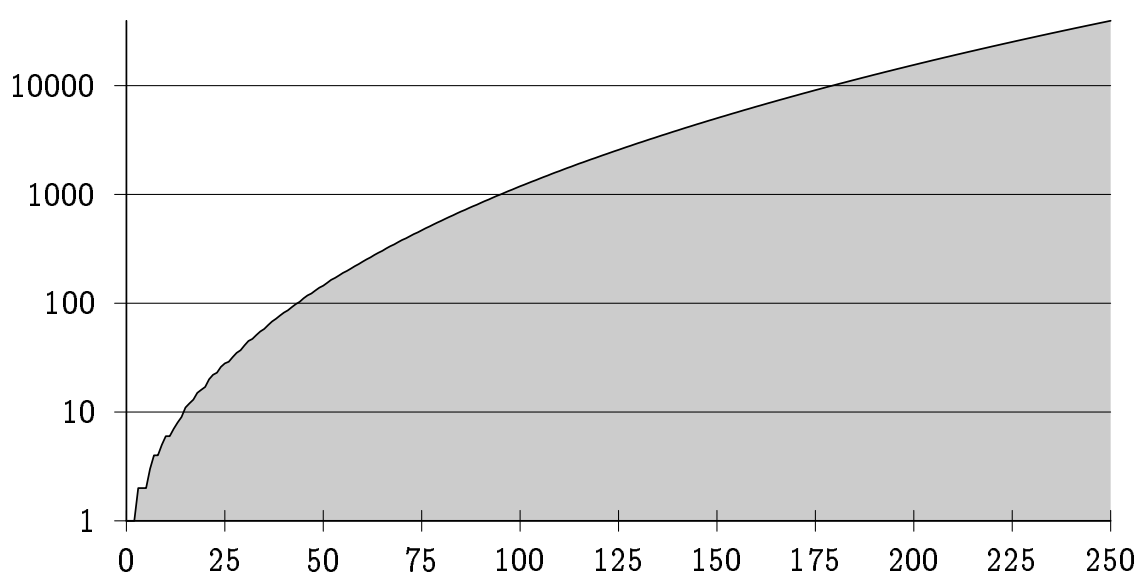

Abbildung 1.6: Dimension der Steenrod-Algebra für $p=2$. Gezeigt wird $\operatorname{dim}_{\mathbb{F}_{2}} A_{n}$ für $n \leq 250$.

Wir beenden das einführende Kapitel mit einer Tabelle. Abbildung 1.7 zeigt den Anfang einer minimalen Auflösung der Steenrod-Algebra zur Primzahl 2, bis zur topologischen Dimension $t-s=14$. Bis hierhin ist es sicherlich noch menschenmöglich, die Auflösung per Hand bzw. auf Papier zu berechnen. In höheren Dimensionen ist das jedoch anders: jedes Erzeugende der Auflösung führt ja eine eigene Kopie der Steenrod-Algebra ein, und diese legt ein nicht unbeträchtliches Wachstum an den Tag. Dies zeigt Abbildung 1.6, in $\operatorname{der} \operatorname{dim} A_{n}$ gegen $n$ für $n \leq 250$ in einem logarithmischen Koordinatensystem dargestellt ist. Man kann zeigen, daß die minimale Auflösung $C_{*}$ als graduierter Vektorraum durch

$$
C_{s} \cong \operatorname{Tor}_{s, *}^{A}(k, k) \otimes_{k} A
$$

beschrieben ist. Die Wachstumsrate von $C_{*}$ wird also sowohl von der Größe von $A$, wie auch von der Größe von $\operatorname{Tor}_{*, *}^{A}(k, k)$ beeinflusst. Ein Blick voraus auf Abbildung 2.14, die die Kenntnis von $\operatorname{Ext}_{A}^{s, t}\left(\mathbb{F}_{2}, \mathbb{F}_{2}\right)$ für $t-s \leq 210$ schon voraussetzt, zeigt, daß man es schnell mit sehr großen Vektorräumen zu tun kriegt. 


\begin{tabular}{|c|c|c|c|}
\hline$n$ & $s$ & $\mathrm{Nr}$. & Differential \\
\hline 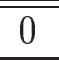 & 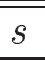 & {$[0]$} & $\overline{\mathrm{Sq}(1)[0]}$ \\
\hline 1 & 1 & {$[1]$} & $\mathrm{Sq}(2)[0]$ \\
\hline 2 & 2 & {$[1]$} & $\mathrm{Sq}(3)[0]+\mathrm{Sq}(2)[1]$ \\
\hline 3 & 3 & {$[1]$} & $\mathrm{Sq}(4)[0]+\mathrm{Sq}(2)[1]+\mathrm{Sq}(1)[2]$ \\
\hline 3 & 2 & {$[2]$} & $\mathrm{Sq}(4)[0]+\mathrm{Sq}(0,1)[1]+\mathrm{Sq}(1)[2]$ \\
\hline 3 & 1 & {$[2]$} & $\mathrm{Sq}(4)[0]$ \\
\hline 6 & 2 & {$[3]$} & $\mathrm{Sq}(1,2)[0]+\mathrm{Sq}(0,2)[1]+\mathrm{Sq}(4)[2]$ \\
\hline 7 & 4 & {$[1]$} & $\mathrm{Sq}(8)[0]+\mathrm{Sq}(2,1)[1]+\mathrm{Sq}(1)[2]$ \\
\hline 7 & 3 & {$[2]$} & $\mathrm{Sq}(8)[0]+\mathrm{Sq}(2,1)[2]+\mathrm{Sq}(1)[4]$ \\
\hline 7 & 2 & {$[4]$} & $\mathrm{Sq}(8)[0]+\mathrm{Sq}(2,1)[2]+\mathrm{Sq}(1)[3]$ \\
\hline 7 & 1 & {$[3]$} & $\mathrm{Sq}(8)[0]$ \\
\hline 8 & 3 & {$[3]$} & $\begin{array}{l}\mathrm{Sq}(3,2)[0]+\mathrm{Sq}(0,0,1)[1]+\mathrm{Sq}(4,1)[1]+\mathrm{Sq}(7)[1]+ \\
\mathrm{Sq}(3,1)[2]+\mathrm{Sq}(6)[2]+\mathrm{Sq}(0,1)[3]+\mathrm{Sq}(3)[3]\end{array}$ \\
\hline 8 & 2 & {$[5]$} & $\begin{array}{l}\mathrm{Sq}(3,2)[0]+\mathrm{Sq}(5,1)[1]+\mathrm{Sq}(8)[1]+\mathrm{Sq}(0,2)[2]+ \\
\mathrm{Sq}(3,1)[2]+\mathrm{Sq}(2)[3]\end{array}$ \\
\hline 9 & 5 & {$[1]$} & $\mathrm{Sq}(10)[0]+\mathrm{Sq}(0,1)[1]+\mathrm{Sq}(3)[1]$ \\
\hline 9 & 4 & {$[2]$} & $\mathrm{Sq}(4,2)[0]+\mathrm{Sq}(0,0,1)[1]+\mathrm{Sq}(1,2)[1]+\mathrm{Sq}(7)[1]+\mathrm{Sq}(2)[3]$ \\
\hline 9 & 3 & {$[4]$} & $\begin{array}{l}\mathrm{Sq}(1,3)[0]+\mathrm{Sq}(5,1)[1]+\mathrm{Sq}(8)[1]+\mathrm{Sq}(4,1)[2]+ \\
\mathrm{Sq}(1,1)[3]+\mathrm{Sq}(4)[3]+\mathrm{Sq}(3)[4]+\mathrm{Sq}(2)[5]\end{array}$ \\
\hline 10 & 6 & {$[1]$} & $\mathrm{Sq}(11)[0]+\mathrm{Sq}(2)[1]$ \\
\hline 11 & 7 & {$[1]$} & $\mathrm{Sq}(12)[0]+\mathrm{Sq}(2)[1]+\mathrm{Sq}(1)[2]$ \\
\hline 11 & 6 & {$[2]$} & $\mathrm{Sq}(12)[0]+\mathrm{Sq}(0,1)[1]+\mathrm{Sq}(1)[2]$ \\
\hline 11 & 5 & {$[2]$} & $\mathrm{Sq}(12)[0]+\mathrm{Sq}(2,1)[1]+\mathrm{Sq}(5)[1]+\mathrm{Sq}(3)[2]$ \\
\hline 14 & 6 & {$[3]$} & $\mathrm{Sq}(9,2)[0]+\mathrm{Sq}(0,2)[1]+\mathrm{Sq}(4)[2]+\mathrm{Sq}(1)[3]$ \\
\hline 14 & 5 & {$[3]$} & $\begin{array}{l}\mathrm{Sq}(3,4)[0]+\mathrm{Sq}(6,3)[0]+\mathrm{Sq}(0,2)[2]+\mathrm{Sq}(3,1)[2]+ \\
\mathrm{Sq}(6)[2]+\mathrm{Sq}(1)[3]\end{array}$ \\
\hline 14 & 4 & {$[3]$} & $\begin{array}{l}\mathrm{Sq}(0,5)[0]+\mathrm{Sq}(6,3)[0]+\mathrm{Sq}(6,2)[1]+\mathrm{Sq}(9,1)[1]+ \\
\mathrm{Sq}(5,1)[2]+\mathrm{Sq}(0,0,1)[3]+\mathrm{Sq}(4,1)[3]+\mathrm{Sq}(7)[3]+ \\
\mathrm{Sq}(3,1)[4]\end{array}$ \\
\hline 14 & 3 & {$[5]$} & $\begin{array}{l}\mathrm{Sq}(0,5)[0]+\mathrm{Sq}(3,4)[0]+\mathrm{Sq}(6,3)[0]+\mathrm{Sq}(6,0,1)[1]+ \\
\mathrm{Sq}(1,4)[1]+\mathrm{Sq}(13)[1]+\mathrm{Sq}(3,3)[2]+\mathrm{Sq}(6,2)[2]+ \\
\mathrm{Sq}(9,1)[2]+\mathrm{Sq}(12)[2]+\mathrm{Sq}(0,3)[3]+\mathrm{Sq}(3,2)[3]+ \\
\mathrm{Sq}(9)[3]+\mathrm{Sq}(2,2)[4]+\mathrm{Sq}(5,1)[4]+\mathrm{Sq}(8)[4]+ \\
\mathrm{Sq}(0,0,1)[5]+\mathrm{Sq}(4,1)[5]+\mathrm{Sq}(7)[5]+\mathrm{Sq}(1)[6]\end{array}$ \\
\hline 14 & 2 & {$[6]$} & $\begin{array}{l}\mathrm{Sq}(0,5)[0]+\mathrm{Sq}(2,4)[1]+\mathrm{Sq}(11,1)[1]+\mathrm{Sq}(0,4)[2]+ \\
\mathrm{Sq}(8)[3]\end{array}$ \\
\hline
\end{tabular}

Abbildung 1.7: Anfang der minimalen Auflösung für $p=2$. Die Tabelle zeigt eine $A$-Basis von $C_{s, t}$ im Bereich $n=t-s \leq 14$. Die Basiselemente jedes $C_{s}$ sind durchnummeriert, beginnend mit $[0] \in C_{s, s}$. Zu jedem $[j] \in C_{s}$ steht in der rechten Spalte das Differential $d_{s}([j])$; die dort auftretenden $\left[n_{i}\right]$ beziehen sich auf $C_{s-1}$. 


\section{Ein Lemma}

Wir kommen zum Herzstück der Arbeit: einem zwar simplen, aber sehr effektiven Lemma, das es uns erlaubt, Redundanzen in der minimalen Auflösung zu erkennen und bei der Berechnung systematisch zu ignorieren. Verschiedene Weisen seiner Anwendung werden vorgestellt. Am Ende werden schließlich - zur Einschüchterung des Lesers und zur Illustration der Stärke unseres Verfahrens - ein paar Kenngrößen der minimalen Auflösung graphisch dargestellt.

\subsection{Das Lemma}

Wie schon angedeutet, besteht ein Problem bei der Berechnung der minimalen Auflösung darin, daß die zu betrachtenden Vektorräume $C_{s, t}$ zu schnell zu groß werden. Wir werden nun zeigen, wie man mithilfe gewisser natürlicher Filtrierungen die $C_{s, t}$ in bekömmlichere Teilstücke zerlegen kann.

\subsubsection{Freundliche Sequenzen}

Wir betrachten zunächst allgemeine graduierte Kettenkomplexe über einem Körper $k$. Zu einer kurzen exakten Sequenz

$$
0 \longrightarrow L_{*} \stackrel{j}{\longrightarrow} M_{*} \stackrel{p}{\longrightarrow} N_{*} \longrightarrow 0
$$

gehört bekanntlich eine lange exakte Sequenz der Homologiegruppen:

$$
\cdots \longrightarrow H_{s, t}\left(L_{*}\right) \stackrel{j_{*}}{\longrightarrow} H_{s, t}\left(M_{*}\right) \stackrel{p_{*}}{\longrightarrow} H_{s, t}\left(N_{*}\right) \stackrel{\partial}{\longrightarrow} H_{s-1, t}\left(L_{*}\right) \longrightarrow \cdots
$$

Definition 2.1.1. Die Sequenz 2.1 heißt $(s, t)$-freundlich, falls $H_{s, t}\left(L_{*}\right)=H_{s-1, t}\left(L_{*}\right)$ $=0$ ist.

Folgende Lemmata sind für die Einstufung "freundlich" verantwortlich. Sie zeigen, daß und wie eine solche Sequenz die Berechnung der Homologie vereinfacht.

Lemma 2.1.2. Die Sequenz 2.1 sei $(s, t)$-freundlich. Dann kann die Homologie $H_{s, t}($ $\left.M_{*}\right)$ folgendermaßen berechnet werden:

1) Berechne die Homologie von

$$
N_{s+1, t} \rightarrow N_{s, t} \rightarrow N_{s-1, t} .
$$

Resultat sind Zykel $n_{1}, \ldots, n_{r} \in N_{s, t}$, die eine Basis von $H_{s, t}\left(N_{*}\right)$ repräsentieren. 
2) Finde Urbilder $m_{i} \in p^{-1}\left(n_{i}\right)$ und bilde $\partial n_{i}:=j^{-1}\left(d m_{i}\right)$.

3) Hebe die $\partial n_{i}$ durch $L_{s, t} \rightarrow L_{s-1, t}$. Resultat sind $l_{i} \in L_{s, t}$ mit $d l_{i}=\partial n_{i}$

Setzt man $\tilde{m}_{i}=m_{i}-j\left(l_{i}\right)$, so sind $\tilde{m}_{1}, \ldots, \tilde{m}_{r}$ Zykel in $M_{s, t}$, die eine Basis von $H_{s, t}\left(M_{*}\right)$ repräsentieren.

Beweis. Zunächst sind die Konstruktionen tatsächlich möglich: die $m_{i}$ lassen sich finden, weil $p$ surjektiv ist; die $l_{i}$ existieren, weil die $\partial n_{i}$ Zykel in $L_{s-1, t}$ sind und $H_{s-1, t}\left(L_{*}\right)$ nach Annahme null ist. Die $\tilde{m}_{i}$ sind tatsächlich Zykel, weil nach Konstruktion $d j\left(l_{i}\right)=j\left(d l_{i}\right)=j\left(j^{-1}\left(d m_{i}\right)\right)=d m_{i}$ gilt.

Aus der langen exakten Homologiesequenz 2.2 folgt sofort, daß $p_{*}$ einen Isomorphismus

$$
H_{s, t}\left(M_{*}\right) \stackrel{\cong}{\longrightarrow} H_{s, t}\left(N_{*}\right)
$$

induziert. Da die $\tilde{m}_{i}$ unter $p$ auf die $n_{i}$ abgebildet werden, repräsentieren sie also eine Basis von $H_{s, t}\left(M_{*}\right)$.

Wir möchten uns an dieser Stelle für die Trivialität dieses Lemmas und die Ausführlichkeit des Beweises entschuldigen. Wir sind nur deshalb so explizit, weil wir die rechnerischen Aspekte noch kurz diskutieren möchten:

- In der Praxis haben $L_{*}, M_{*}$ und $N_{*}$ bevorzugte, explizit bekannte Vektorraumbasen. Alle Abbildungen und alle Vektoren sind für uns also Matrizen.

- Die Berechnung der Homologie der Sequenz 2.3 ist Standard: man berechnet ker $d: N_{s, t} \rightarrow N_{s-1, t}$ und reduziert dies modulo im $d: N_{s+1, t} \rightarrow N_{s, t}$. Dabei fallen die geforderten Zykel $n_{1}, \ldots, n_{r} \in N_{s, t}$ von alleine an.

- Die Berechnung der Urbilder $p^{-1}(\cdots)$ und $j^{-1}(\cdots)$ soll als einfach gelten. In unseren Anwendungen kann man dies vernachlässigen.

- Dasselbe gelte für die Berechnung von $d m_{i}$ bei gegebenen $m_{i}$. Wir vernachlässigen dies, weil die Berechnung der $d m_{1}, \ldots, d m_{r}$ gegenüber der andernfalls nötigen Berechnung der ganzen Matrix von $d: M_{s, t} \rightarrow M_{s-1, t}$ nicht ins Gewicht fällt. $r$ ist nämlich bei uns wesentlich kleiner als $\operatorname{dim} M_{s, t}$ und $\operatorname{dim} M_{s-1, t}$.

- Die Hebung der $\partial n_{i}$ durch $d: L_{s, t} \rightarrow L_{s-1, t}$ wird ebenfalls mit dem Standardverfahren berechnet.

Um die Vorteile dieser Methode kenntlich zu machen, wählen wir Zerlegungen $M_{s, t} \cong$ $L_{s, t} \oplus N_{s, t}$. Man stelle sich vor, daß $L_{s, t}$ und $N_{s, t}$ in etwa dieselbe Dimension $D / 2$ haben, $D \gg 0$. Das Differential $d_{s, t}^{M}: M_{s, t} \rightarrow M_{s-1, t}$ schreibt sich dann in Matrixform als

$$
d_{s, t}^{M}=\left(\begin{array}{cc}
d_{s, t}^{N} & X_{s, t} \\
0 & d_{s, t}^{L}
\end{array}\right)
$$

wobei $d^{L}$ und $d^{N}$ die Differentiale von $L_{*}$ respektive $N_{*}$ sind. Man sieht, daß bei obigem Rechenverfahren nur $d_{s+1, t}^{N}, d_{s, t}^{N}$ und $d_{s, t}^{L}$ benutzt wurden, und zwar nacheinander. $X_{s, t}$ wird implizit bei der Berechnung der $d m_{i}$ mitbenutzt, muß aber nie vollständig als Matrix berechnet werden. Es ergeben sich also 
- ein geringerer Speicherplatzbedarf: $D^{2} / 4$ statt $D^{2}$,

- schnellere Laufzeiten für die Berechnung von Kern und Bild.

Das Standardverfahren zur Berechnung eines Kerns hat eine Laufzeit, die in etwa proportional zu $D^{3}$ ist. Der Übergang von $D$ zu $D / 2$ ist also spürbar.

Auch Hebungsprobleme lassen sich in diesem Fall leichter lösen:

Lemma 2.1.3. Die Sequenz 2.1 sei $(s, t)$-freundlich, und es sei $H_{s, t}\left(M_{*}\right)=0$. Ist ein Zykel $z \in M_{s, t}$ gegeben, so kann man einen Lift $m \in M_{s+1, t}$ mit $d m=z$ so berechnen:

1) Bilde $p(z) \in N_{s, t}$ und berechne einen Lift $n \in N_{s+1, t}$ mit $d n=p(z)$.

2) Wähle $\tilde{m} \in p^{-1}(n)$ und bilde $l=j^{-1}(d \tilde{m}-z) \in L_{s, t}$.

3) Berechne einen Lift $\tilde{l} \in L_{s+1, t}$ mit $d \tilde{l}=l$.

Dann ist $m:=\tilde{m}-j(\tilde{l})$ eine Hebung von $z$.

Beweis. Die Konstruktion ist möglich: Wegen $H_{s, t}\left(M_{*}\right) \cong H_{s, t}\left(N_{*}\right)$ ist auch $H_{s, t}\left(N_{*}\right)$ $=0$, sodaß der Zykel $p(z)$ einen Lift $n$ besitzt; $\tilde{m}$ existiert, weil $p$ surjektiv ist; wegen $p(z-d \tilde{m})=p(z)-d n=0$ existiert $l ;$ da $j(d l)=d(z-d \tilde{m})=0$, ist $d l=0$; wegen $H_{s, t}\left(L_{*}\right)=0$ existiert also auch $\tilde{l}$. Und die Konstruktion führt zum Erfolg: schließlich ist $d m=d \tilde{m}-j(d \tilde{l})=d \tilde{m}-j(l)=d \tilde{m}+z-d \tilde{m}=z$, wie gewollt.

Auch hier schließen wir eine kurze Diskussion an:

- Die Bildung der diversen Urbilder und die Berechnung von $d \tilde{m}$ aus $\tilde{m}$ können wir wieder vernachlässigen.

- Man hat hier das Hebungsproblem zu $d_{s, t}^{M}$ durch die sukzessive Hebung durch $d_{s, t}^{N}$ und $d_{s, t}^{L}$ ersetzt. Die Vorteile betreffs Speicherplatzbedarf und Laufzeit sind also dieselben wie oben.

\subsubsection{Das Lemma}

Wir untersuchen hier, wie die Existenz "verallgemeinerter Margolis-Differentiale" zu freundlichen Sequenzen führt.

$M_{*}$ sei ein graduierter Kettenkomplex aus $k$-Vektorräumen. Wir betrachten die folgende Situation:

- Gegeben sei eine Kettenabbildung $Q: M_{*, *} \rightarrow M_{*, *+q}$ mit $Q^{l}=0$ für ein $l>1$.

Setze $D=k[Q] /\left(Q^{l}\right)$.

- $M_{*}$ sei als $D$-Modul frei.

Natürlich auftretende Beispiele findet man im nächsten Abschnitt. Wir bemerken im Augenblick nur dreierlei: 
1) Ist $l=2$, so ist $Q$ gerade ein Differential auf $M_{*}$, und die Voraussetzung sagt einfach, daß $H\left(M_{*}, Q\right)=0$ sein soll. Für $l>2$ ist man also versucht, $Q$ als "verallgemeinertes Differential" zu betrachten.

2) Ist char $k=l$, so ist $D$ zum Gruppenring $k \mathfrak{C}_{l}$ mit $\mathfrak{C}_{l}=\left\langle T \mid T^{l}=1\right\rangle$ isomorph, via $Q \leftrightarrow T-1 . Q$ kann dann als eine freie Operation von $\mathfrak{C}_{l}$ aufgefasst werden.

3) In der Steenrod-Algebra wimmelt es von solchen verallgemeinerten Differentialen, deren Bedeutung vor allem von Margolis untersucht wurde. Siehe [29], Part II.

Wir bezeichnen den Kettenkomplex $\operatorname{im}\left(Q^{k}: M_{*, *-k q} \rightarrow M_{*, *}\right)$ mit $Q^{k} M_{*}$. Die Graduierung auf $Q^{k} M_{*}$ sei so gewählt, daß $Q^{k}: M_{*} \rightarrow Q^{k} M_{*}$ graderhaltend ist; die Inklusion $Q^{k} M_{*} \subset M_{*}$ erhöht damit den Grad um $k q$.

Lemma 2.1.4. Für jedes $0 \leq k^{\prime} \leq k \leq l$ ist

$$
0 \rightarrow Q^{k} M_{*, *-\left(k-k^{\prime}\right) q} \stackrel{\text { incl. }}{\longrightarrow} Q^{k^{\prime}} M_{*, *} \stackrel{Q^{l-k}}{\longrightarrow} Q^{l-\left(k-k^{\prime}\right)} M_{*, *} \rightarrow 0
$$

exakt.

Beweis. $0 \rightarrow Q^{k} D \stackrel{\text { incl. }}{\longrightarrow} Q^{k^{\prime}} D \stackrel{Q^{l-k}}{\longrightarrow} Q^{l-\left(k-k^{\prime}\right)} D \rightarrow 0$ ist exakt und 2.4 ist eine direkte Summe solcher Sequenzen, weil $M_{*}$ über $D$ frei ist.

Der folgende Spezialfall ist besonders wichtig: für jedes $k \leq l$ ist

$$
0 \rightarrow Q^{k} M_{*, *-k q} \stackrel{\text { incl. }}{\longrightarrow} M_{*, *} \stackrel{Q^{l-k}}{\longrightarrow} Q^{l-k} M_{*, *} \rightarrow 0
$$

exakt. Man kann den allgemeinen Fall auch hierauf zurückführen, denn

Lemma 2.1.5. Ist $k \leq l$ und setzt man $\tilde{M}_{*}=Q^{k} M_{*}, \tilde{l}=l-k, \tilde{Q}=Q$ und $\tilde{D}=k[\tilde{Q}] /\left(\tilde{Q}^{\tilde{l}}\right)$, so ist $\stackrel{\tilde{M}}{*}_{*}$ als $\tilde{D}$-Modul frei.

Beweis. Klar, denn $Q^{k} D$ ist ein freier $k[Q] /\left(Q^{l-k}\right)$-Modul.

Wir möchten nun zu folgendem Problem ein systematisches Resultat formulieren:

Problem 2.1.6. Angenommen $M_{*}$ sei in einem bekannten Bereich $R \subset \mathbb{Z}^{2}$ exakt. Wann und für welche $k$ gilt dasselbe für $Q^{k} M_{*}$ ? Wann ist die Sequenz $2.5(s, t)$ freundlich, und für welche $(s, t)$ ?

Alle Resultate hierzu folgen aus einer simplen Diagrammjagd in der langen exakten Homologiesequenz zu 2.5:

$$
\begin{gathered}
\cdots \rightarrow H_{s, t}\left(M_{*}\right) \stackrel{Q_{*}^{l-k}}{\longrightarrow} H_{s, t}\left(Q^{l-k} M_{*}\right) \stackrel{\partial}{\longrightarrow} H_{s-1, t}\left(Q^{k} M_{*, *-k q}\right) \stackrel{\text { incl.* }}{\longrightarrow} H_{s-1, t}\left(M_{*}\right) \rightarrow \cdots \\
\| \\
H_{s-1, t-k q}\left(Q^{k} M_{*}\right)
\end{gathered}
$$


Lemma 2.1.7. 1) Ist $H_{s, t}\left(M_{*}\right)=H_{s-1, t}\left(M_{*}\right)=0$, so ist $H_{s, t}\left(Q^{l-k} M_{*}\right) \cong H_{s-1, t-k q}($ $\left.Q^{k} M_{*}\right)$.

2) Ist außerdem $H_{s-1, t-k q}\left(M_{*}\right)=H_{s-2, t-k q}\left(M_{*}\right)=0$, so ist $H_{s, t}\left(Q^{l-k} M_{*}\right) \cong$ $H_{s-2, t-l q}\left(Q^{l-k} M_{*}\right)$.

Beweis. 1) ist klar. 2) folgt aus zweimaliger Anwendung von 1), wobei beim zweiten Mal $k$ durch $l-k$ ersetzt wird.

Eine graphische Darstellung ist vielleicht nützlich. Im Bild ist $q=1, k=2, l=5$ :

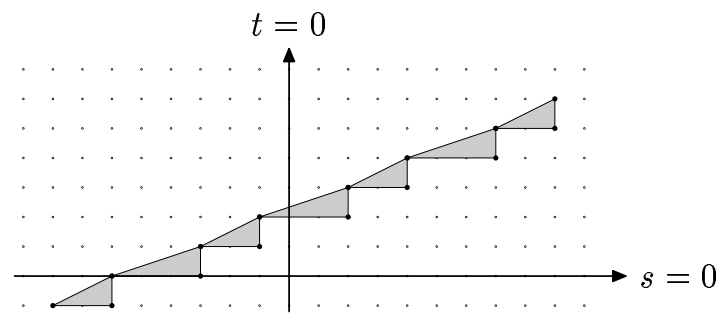

Die Diagrammjagd beginnt in der rechten oberen Ecke und arbeitet sich nach unten durch. Voraussetzung ist, daß $M_{*}$ in den Ecken exakt ist. Gefolgert wird, daß die Homologiegruppen von $Q^{l-k} M_{*}$ und $Q^{k} M_{*}$, jeweils abwechselnd in den Punkten der oberen Kante gebildet, isomorph sind. Und der springende Punkt ist, daß man dies unter den richtigen Voraussetzungen bis zu negativen homologischen Graden fortsetzen kann. Wenn unsere $M_{*}$ dort null sind, verschwinden also die $H\left(Q^{k} M_{*}\right)$ und $H\left(Q^{l-k} M_{*}\right): 2.4$ und 2.5 sind dann für passende $(s, t)$ freundliche Sequenzen. Details folgen:

Sei $\rho>0, r \in \mathbb{R} . A^{\rho, r+}$ und $A^{\rho, r-}$ seien die offenen Halbebenen

$$
\begin{aligned}
& A^{\rho, r+}=\left\{(s, t) \in \mathbb{Z}^{2} \mid t-r<s \cdot \rho\right\}, \\
& A^{\rho, r-}=\left\{(s, t) \in \mathbb{Z}^{2} \mid t-r>s \cdot \rho\right\},
\end{aligned}
$$

mit Steigung $1 / \rho$.

Das Lemma 2.1.8 (obere Version). Sei $M_{s}=0$ für $s<0$. Seien $S, T \in \mathbb{Z}$. Es gelte $H_{s, t}\left(M_{*}\right)=0$ für alle $(s, t) \in R_{S, T}^{o} \cap A^{\rho, r+}$. Die Steigung $1 / \rho$ erfülle $\rho \leq l q / 2$. Setze $r^{\prime}=\min \{r-\rho, r-2 \rho+(l-k) q\}$. Dann gilt:

1) $H_{s, t}\left(Q^{k} M_{*}\right)=0$ für $(s, t) \in A^{\rho, r^{\prime}+} \cap R_{S, T}^{o}$.

2) Die Sequenz 2.5 ist $(s, t)$-freundlich für $(s, t) \in A^{\rho,\left(r^{\prime}+k q-\rho\right)+} \cap R_{S, T+k q}^{o}$.

Beweis. $r^{\prime}$ ist gerade so gewählt, daß für $(s, t) \in A^{\rho, r^{\prime}+}$ jeweils $(s, t),(s-1, t)$ und $(s-2, t-(l-k) q)$ in $A^{\rho, r+}$ liegen; Lemma 2.1.7 ist deswegen beliebig oft rekursiv anwendbar und liefert

$$
H_{s, t}\left(Q^{k} M_{*}\right) \cong H_{s-2, t-l q}\left(Q^{k} M_{*}\right) \cong \ldots \cong H_{s-2 j, t-l j q}\left(Q^{k} M_{*}\right), \quad j \geq 0 \text { beliebig. }
$$

Wählt man $j>s / 2$, so folgt $H_{s, t}\left(Q^{k} M_{*}\right)=0$, wie in 1) behauptet. Siehe auch Abbildung 2.1.

$2)$ ist ein Korollar: für $(s, t) \in A^{\rho,\left(r^{\prime}+k q-\rho\right)+} \cap R_{S, T+k q}^{o}$ liegen $(s, t-k q)$ und $(s-$ $1, t-k q)$ in $A^{\rho, r^{\prime}+} \cap R_{S, T}^{o}$, sodaß $H_{s, t-k q}\left(Q^{k} M_{*}\right)$ und $H_{s-1, t-k q}\left(Q^{k} M_{*}\right)$ null sind. 


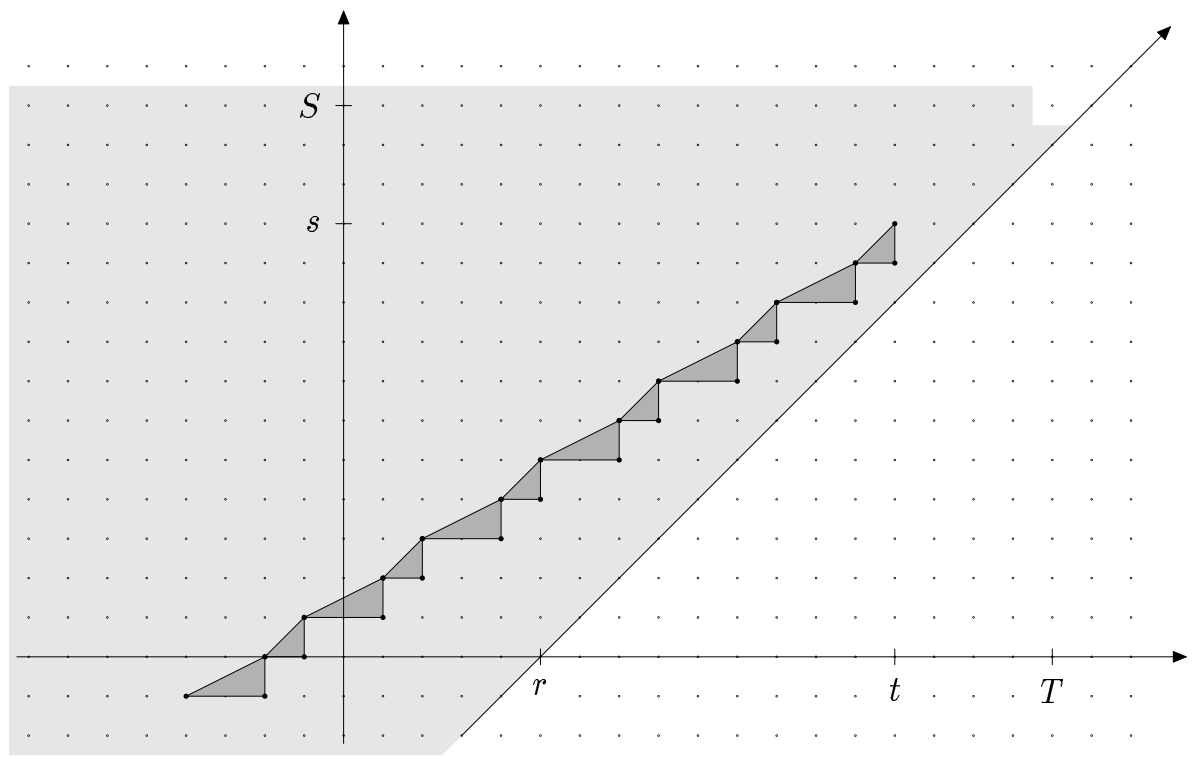

Abbildung 2.1: Ein Bild zur oberen Version des Lemmas; hier ist $q=1, l=3, k=2$, $\rho=1, r=5$. Die Steigung des Dreieckswegs ist $2 / l q$. Wegen $2 / l q \leq 1 / \rho$ liegt der ganze Weg im guten Bereich, falls nur $(s, t),(s-1, t)$ und $(s-2, t-(l-k) q)$ dort liegen.

Man beachte, daß unter geeigneten Umständen $(S, T)$ selber in $A^{\rho,\left(r^{\prime}-k q+\rho\right)+} \cap$ $R_{S, T+k q}^{o}$ liegt; 2.4 ist dann also $(S, T)$-freundlich, obwohl $M_{*}$ in $(S, T)$ nicht exakt zu sein braucht.

Das Lemma 2.1 .9 (untere Version). Sei $M_{s}=0$ für $s<0$. Seien $S, T \in \mathbb{Z}$. Es gelte $H_{s, t}\left(M_{*}\right)=0$ für alle $(s, t) \in R_{S, T}^{o} \cap A^{\rho, r-}$. Die Steigung $1 / \rho$ erfülle $\rho \geq l q / 2$. Setze $r^{\prime}=\max \{r, r+(l-k) q-\rho\}$. Dann gilt:

1) $H_{s, t}\left(Q^{k} M_{*}\right)=0$ für $(s, t) \in A^{\rho, r^{\prime}-} \cap R_{S, T}^{o}$.

2) Die Sequenz 2.5 ist $(s, t)$-freundlich für $(s, t) \in A^{\rho,\left(r^{\prime}+k q\right)-} \cap R_{S, T+k q}^{o}$.

Beweis. $r^{\prime}$ ist gerade so gewählt, daß für $(s, t) \in A^{\rho, r^{\prime}-}$ jeweils $(s, t)$ und $(s-1, t-$ $(l-k) q)$ in $A^{\rho, r-}$ liegen; also ist Lemma 2.1.7 beliebig oft anwendbar und wie oben ist

$$
H_{s, t}\left(Q^{k} M_{*}\right) \cong H_{s-2, t-l q}\left(Q^{k} M_{*}\right) \cong \ldots \cong 0 .
$$

Siehe auch Abbildung 2.2.

2) ist ein Korollar: für $(s, t) \in A^{\rho,\left(r^{\prime}+k q\right)-} \cap R_{S, T+k q}^{o}$ liegen $(s, t-k q)$ und $(s-$ $1, t-k q)$ in $A^{\rho, r^{\prime}-} \cap R_{S, T}^{o}$, sodaß $H_{s, t-k q}\left(Q^{k} M_{*}\right)$ und $H_{s-1, t-k q}\left(Q^{k} M_{*}\right)$ null sind.

\subsection{Weisen seiner Anwendung}

Wir zeigen jetzt, wie man die beiden Varianten des Lemmas bei der Berechnung der minimalen Auflösung der Steenrod-Algebra einsetzen kann. 


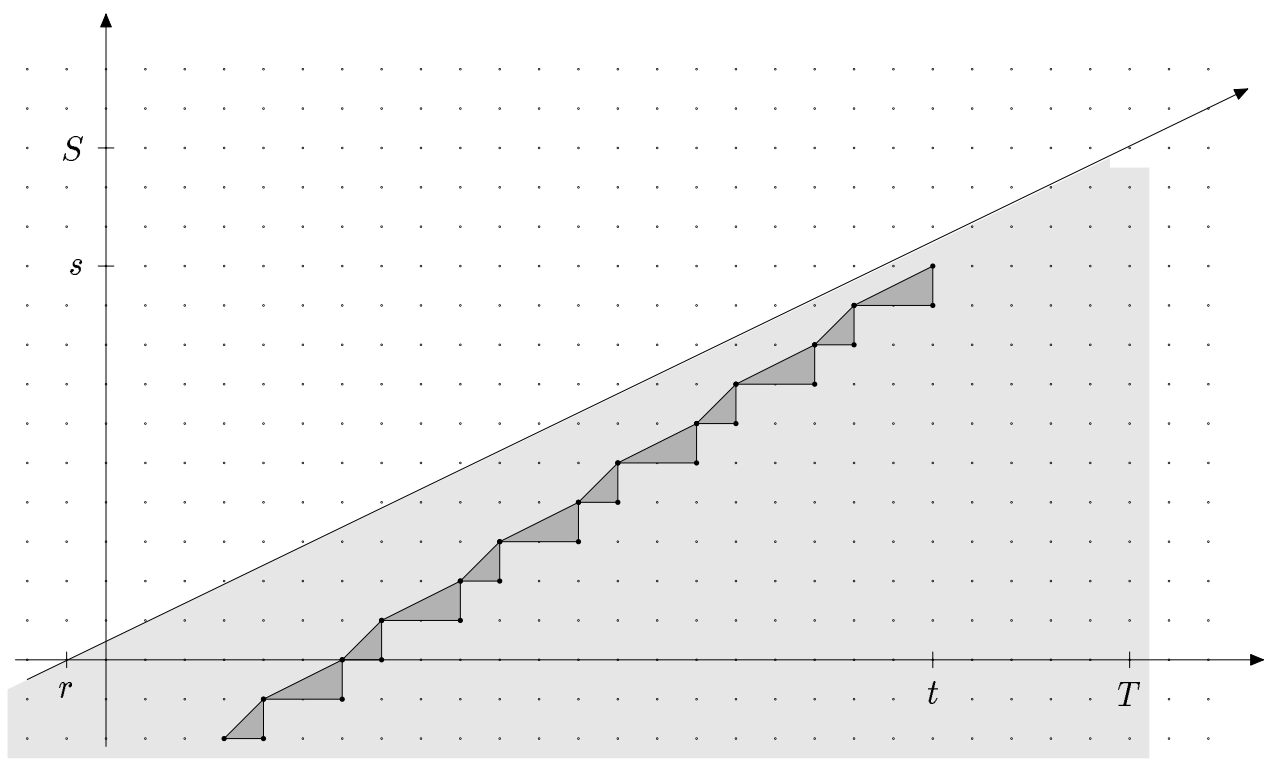

Abbildung 2.2: Ein Bild zur unteren Version des Lemmas. Hier sei $\rho \geq l q / 2$. Die Diagrammjagd längs des Dreieckswegs ist möglich, sobald $(s, t)$ und $(s-1, t-(l-k) q)$ in $A^{\rho, r-}$ liegen.

\subsubsection{Von Unten, zum Aufwärmen}

$A$ sei die Steenrod-Algebra zur Primzahl $p . C_{*}$ sei ein Zwischenschritt bei der Berechnung der minimalen Auflösung von $A$. In den Bezeichnungen von Abschnitt 1.3.2 seien also $S, T \geq 0$ und $C_{*}$ in $(S, T)$ erweiterbar. Wir benutzen den klassischen

Satz 2.2.1 (Milnor-Moore). Ist A eine Hopf-Algebra, $B \subset A$ eine Hopf-Unteralgebra, so ist $A$ als $B$-Modul frei.

Beweis. Für eine Inklusion von Gruppenalgebren $k H \subset k G$ ist dies klar: als $k H$ Modul ist $k G=\oplus k N_{g}$, wobei über die Nebenklassen $N_{g}=H g$ summiert wird. Da $h \mapsto h g$ jeweils eine $H$-äquivariante Bijektion $H \cong N_{g}$ ist, sind die $k N_{g}$ freie $k H$-Moduln. Den allgemeinen Fall findet man in [35].

Die äußere Algebra $E\left(Q_{0}, Q_{1}, \ldots\right) \subset A$ ist eine Hopf-Unteralgebra, weil jedes $Q_{i}$ primitiv ist. Nach Satz 2.2.1 ist $A$ also ein freier $E\left(Q_{0}, Q_{1}, \ldots\right)$-Modul. Deswegen sind folgende Sequenzen exakt:

$$
\begin{gathered}
0 \longrightarrow Q_{0} C_{*, *-1} \stackrel{\text { incl. }}{\longrightarrow} C_{*, *} \stackrel{Q_{0}}{\longrightarrow} Q_{0} C_{*, *} \longrightarrow 0 \\
0 \longrightarrow Q_{1} Q_{0} C_{*, *-(2 p-1)} \stackrel{\text { incl. }}{\longrightarrow} Q_{0} C_{*, *} \stackrel{Q_{1}}{\longrightarrow} Q_{1} Q_{0} C_{*, *} \longrightarrow 0 \\
0 \longrightarrow Q_{2} Q_{1} Q_{0} C_{*, *-\left(2 p^{2}-1\right)} \stackrel{\text { incl. }}{\longrightarrow} Q_{1} Q_{0} C_{*, *} \stackrel{Q_{2}}{\longrightarrow} Q_{2} Q_{1} Q_{0} C_{*, *} \longrightarrow 0 \\
\vdots \\
0 \rightarrow Q_{n} \cdots Q_{0} C_{*, *-\left(2 p^{n}-1\right)} \stackrel{\text { incl. }}{\longrightarrow} Q_{n-1} \cdots Q_{0} C_{*, *} \stackrel{Q_{n}}{\longrightarrow} Q_{n} \cdots Q_{0} C_{*, *} \rightarrow 0
\end{gathered}
$$

Lemma 2.1.9 sagt hier: 


\section{Ein Lemma}

Korollar 2.2.2. Es ist $H_{s, t}\left(Q_{n} \cdots Q_{0} C_{*, *}\right)=0$ für $(s, t) \in A^{2 p^{n}-1,0-} \cap R_{S, T}^{o}$.

Beweis. Durch Induktion über $n$. Zur Verankerung betrachten wir $n=-1$; dort wird gerade $H_{s, t}\left(C_{*}\right)=0$ für $(s, t) \in R_{S, T}^{o} \backslash\{(0,0)\}$ behauptet, was wir aber einfach gefordert haben.

Zum Induktionsschritt $n \Rightarrow n+1$ : Wir setzen $M_{*}=Q_{n} \cdots Q_{0} C_{*}, Q=Q_{n+1}, q=$ $2 p^{n+1}-1, l=2, k=1, r=0$. Nach Annahme ist $M_{*}$ in $A^{2 p^{n}-1,0-} \cap R_{S, T}^{o}$ exakt. Wir dürfen die Steigung $1 / \rho$ sicher verkleinern und setzen also $\rho=2 p^{n+1}-1>2 p^{n}-1$. Es ergibt sich $r^{\prime}=r=0$, sodaß $H_{s, t}\left(Q_{n+1} \cdots Q_{0} C_{*}\right)=0$ für $(s, t) \in A^{2 p^{n+1}-1,0-} \cap R_{S, T}^{o}$, wie behauptet.

Bemerkung 2.2.3 (zwecks Demystifikation). Angenommen $C_{*}$ wäre eine vollständige Auflösung von $k$ über $A$. Wegen Satz 2.2.1 wäre $C_{*}$ dann auch eine $E$-freie Auflösung von $k$ mit $E=E\left(Q_{0}, \ldots, Q_{n}\right)$. Die Homologie $H\left(Q_{n} \cdots Q_{0} C_{*}\right)$ berechnet dann die derivierten Funktoren zu

$$
\begin{aligned}
E \text {-Mod } & \rightarrow k \text {-Mod, } \\
M & \mapsto Q_{n} \cdots Q_{0} M .
\end{aligned}
$$

Man kann aber $Q_{n} \cdots Q_{0} M$ mit $k \otimes_{E} M$ vermöge

$$
k \otimes_{E} M \ni \lambda \otimes m \longmapsto Q_{n} \cdots Q_{0} \lambda \otimes m \in Q_{n} \cdots Q_{0} E \otimes_{E} M=Q_{n} \cdots Q_{0} M
$$

identifizieren, weil $\lambda \mapsto Q_{n} \cdots Q_{0} \lambda$ ein Isomorphismus $k \rightarrow Q_{n} \cdots Q_{0} E$ von $E$ Moduln ist. Also ist dann $H_{s, t}\left(Q_{n} \cdots Q_{0} C_{*}\right)=\operatorname{Tor}_{s, t}^{E}(k, k)$. Bekanntlich ist aber Tor über einer äußeren Algebra die entsprechende Polynomalgebra, also

$$
\operatorname{Tor}_{s, t}^{E}(k, k)=P\left(v_{0}, \ldots, v_{n}\right) \quad \text { mit } v_{i} \in \operatorname{Tor}_{1,2 p^{i}-1}^{E}(k, k) .
$$

$A^{2 p^{n}-1,0-}$ ist gerade der Bereich, in dem $P\left(v_{0}, \ldots, v_{n}\right)$ offensichtlich null ist. Korrolar 2.2.2 sagt nur, daß dies unter unseren eingeschränkten Voraussetzungen noch partiell richtig bleibt.

Man erinnere sich an den Erweiterungsschritt auf Seite 23. Das folgende Korollar sagt, daß zumindest der erste Teilschritt vereinfacht werden kann:

Korollar 2.2.4. $H_{S, T}\left(C_{*}\right) \rightarrow H_{S, T}\left(Q_{n} \cdots Q_{0} C_{*}\right)$ ist für $T>\left(2 p^{n}-1\right) S$ ein Isomorphismus.

Beweis. Durch Induktion nach $n$. Der Fall $n=-1$ ist wieder trivial. Für $n-1 \Rightarrow n$ ist zu zeigen, daß

$$
H_{S, T}\left(Q_{n-1} \cdots Q_{0} C_{*}\right) \stackrel{Q_{n_{*}}}{\longrightarrow} H_{S, T}\left(Q_{n} \cdots Q_{0} C_{*}\right)
$$

ein Isomorphismus ist. Mithilfe der langen exakten Sequenz zu 2.10 folgt dies, wenn sowohl $H_{S, T-\left(2 p^{n}-1\right)}\left(Q_{n} \cdots Q_{0} C_{*}\right)$ wie $H_{S-1, T-\left(2 p^{n}-1\right)}\left(Q_{n} \cdots Q_{0} C_{*}\right)$ null sind. Da $(S$, $\left.T-\left(2 p^{n}-1\right)\right) \in R_{S, T}^{o}$, ist Korollar 2.2.2 anwendbar. Die dort geforderte Bedingung ist gerade unser $T-\left(2 p^{n}-1\right) S>0$. 
Damit kann man also den ersten Teilschritt des Erweiterungsschritts im Bereich $T-\left(2 p^{n}-1\right)(S-1)>0$ einfacher lösen: man kann in $Q_{n} \cdots Q_{0} C_{*}$ statt in $C_{*}$ rechnen. Den zweiten Teilschritt möchten wir durch wiederholte Anwendung von Lemma 2.1.2 und Lemma 2.1.3 vereinfachen. Dazu müssen die Sequenzen 2.7, . ,2.10 in allen betrachteten Bigraden freundlich sein. Zur Erinnerung: Lemma 2.1.2 ersetzt eine Homologieberechnung in $Q_{i} \cdots Q_{0} C_{*}$ im Bigrad $(s, t)$ durch eine Homologieberechnung in $Q_{i+1} \cdots Q_{0} C_{*}$ im Bigrad $(s, t)$ und eine Hebung durch $Q_{i+1} \cdots Q_{0} C_{*}$ im Bigrad $\left(s, t-\left|Q_{i+1}\right|\right)$; in symbolischer Notation:

$$
H_{s, t}\left(Q_{i} \cdots Q_{0} C_{*}\right) \stackrel{\text { Lemma 2.1.2 }}{=} H_{s, t}\left(Q_{i+1} \cdots Q_{0} C_{*}\right)+L_{s, t-q_{i+1}}\left(Q_{i+1} \cdots Q_{0} C_{*}\right) .
$$

Hier steht $L$ für Lift und wir haben zur Abkürzung $q_{i}=\left|Q_{i}\right|$ gesetzt. In derselben Notation gilt auch

$$
L_{s, t}\left(Q_{i} \cdots Q_{0} C_{*}\right) \stackrel{\text { Lemma } 2.1 .3}{=} L_{s, t}\left(Q_{i+1} \cdots Q_{0} C_{*}\right)+L_{s, t-q_{i+1}}\left(Q_{i+1} \cdots Q_{0} C_{*}\right) .
$$

Durch Iteration ergibt sich also folgender Baum:

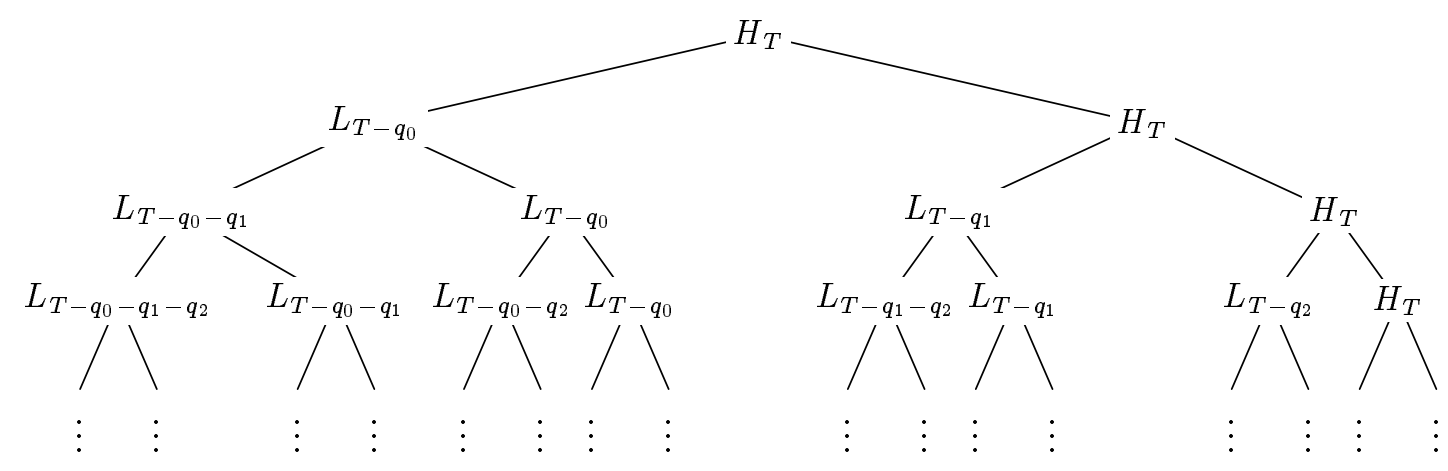

Die Rechnung beginnt dabei mit der Homologieberechnung am rechten Ende und arbeitet sich nach links durch. Wie man sieht, müssen wir uns auf die Freundlichkeit in den Bigraden $\left(S, T^{\prime}\right)$ mit $T^{\prime}$ zwischen $T$ und $T-\tau_{n}$ verlassen können, wobei wir

$$
\tau_{n}=\left|Q_{0}\right|+\cdots+\left|Q_{n}\right|=2 \frac{p^{n+1}-1}{p-1}-(n+1)
$$

setzen. $\tau_{n}$ ist die höchste Dimension, in $\operatorname{der} E\left(Q_{0}, \ldots, Q_{n}\right)$ von Null verschieden ist.

Satz 2.2.5. Ist $T-\left(2 p^{n}-1\right) S>\tau_{n}$, so sind 2.7,..,2.10 in allen relevanten Bigraden freundlich.

Beweis. Wie gerade gesehen, müssen dazu $H_{S, T^{\prime}}\left(Q_{n} \cdots Q_{0} C_{*}\right)$ und $H_{S-1, T^{\prime}}\left(Q_{n} \cdots\right.$ $\left.Q_{0} C_{*}\right)$ für $T-\tau_{n} \leq T^{\prime}<T$ null sein. Aus Korollar 2.2 .2 wissen wir, daß dies für $\left(S, T-\tau_{n}\right) \in A^{2 p^{n}-1,0-}$, dh. $T-\tau_{n}>\left(2 p^{n}-1\right) S$ der Fall ist.

\subsubsection{Zulässige Unteralgebren}

Die Methode des vergangenen Abschnitts läßt sich noch ausbauen. Statt $E\left(Q_{0}, \ldots\right.$, $\left.Q_{n}\right)$ kann man allgemeinere endliche Hopf-Unteralgebren $B \subset A$ betrachten. Die dabei zulässigen $B$ werden in diesem Abschnitt untersucht. 


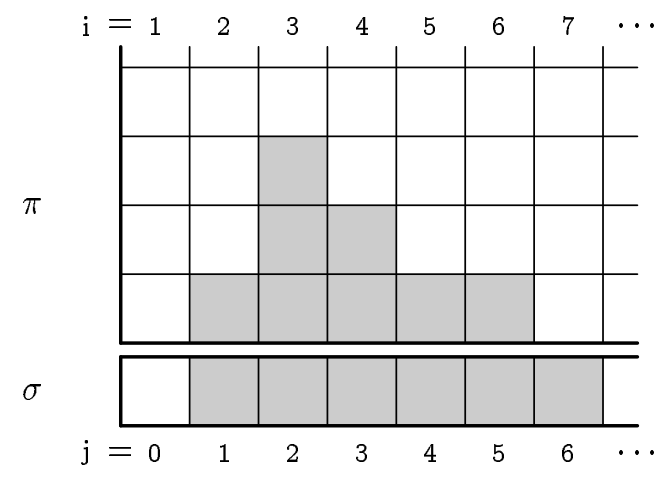

Abbildung 2.3: Graphische Darstellung einer Profilfunktion. Die $j$ mit $\sigma(j) \neq 1$ und die Paare $(i, y)$ mit $1 \leq y \leq \pi(i)$ werden durch geschwärzte Kästchen dargestellt. Im Beispiel ist $\pi=(0,1,3,2,1,1,0, \ldots)$ und $\sigma=(1,2,2,2,2,2,2,1,1, \ldots)$. Das zugehörige $B(\pi, \sigma)$ ist also zulässig.

Wir beschränken uns in der Darstellung auf den Fall $p>2$ und benutzen die Klassifikation solcher Unteralgebren aus [3], die wir in Abschnitt 1.1.4 wiedergegeben haben. Für $p=2$ ignoriert man einfach alle Bezüge auf die $\sigma$-Komponenten der auftretenden Profilfunktionen. Sei $\Pi=(\pi, \sigma)$ mit

$$
\pi:\{1,2, \ldots\} \rightarrow\{0,1,2, \ldots, \infty\}, \quad \sigma:\{0,1,2, \ldots\} \rightarrow\{1,2\}
$$

eine Profilfunktion und $B(\Pi)$ die zugehörige Hopf-Unteralgebra von $A$. Wir nennen $\Pi$ und $B(\Pi)$ zulässig, wenn folgende Verschärfungen von 1.1 und 1.2 (siehe Seite 9) erfüllt sind:

Für alle $i, j \geq 1$ gilt $\pi(i) \leq j+\pi(i+j)$.

Für alle $i \geq 1, j \geq 0$ mit $\sigma(i+j)=1$ gilt $\pi(i) \leq j$.

Abbildung 2.3 zeigt eine graphische Darstellung einer zulässigen Profilfunktion.

Wir betrachten nur endliche zulässige Hopf-Unteralgebren $B=B(\Pi)$. Für ein solches $B$ sind folgende Größen definiert:

- Das Element höchster Dimension $T_{B} \in B$.

- Der Grad von $T_{B}: \tau_{B}=\left|T_{B}\right|$.

- Die maximale inverse Steigung

$$
d_{B}=\max \left(\left\{(p-1) \cdot 2\left(p^{i}-1\right) \cdot p^{\pi(i)-1}: \pi(i) \neq 0\right\} \cup\left\{\left|Q_{j}\right|: Q_{j} \in B\right\}\right) .
$$

$T_{B}$ ist nur bis auf einen Koeffizienten aus $\mathbb{F}_{p}^{*}$ bestimmt. Die naheliegende Wahl ist

$$
T_{B}=\left(\prod_{j \text { mit } \sigma(j)=2} Q_{j}\right) \cdot P\left(p^{\pi(1)}-1, p^{\pi(2)}-1, p^{\pi(3)}-1, \ldots\right) .
$$

Wir schreiben $Q\left(\tilde{\varepsilon}_{B}\right) P\left(\tilde{R}_{B}\right)$ für dieses Element. Den Index $B$ lassen wir weg, wenn er aus dem Zusammenhang ersichtlich ist. 
Definition 2.2.6. Sei $B=B(\Pi)$ eine Hopf-Unteralgebra, $Q(\varepsilon) P(R) \in A$ ein Milnorsches Basiselement. Unter der B-Signatur $\operatorname{sig}_{B}(Q(\varepsilon) P(R))$ verstehen wir

$$
\left(\varepsilon_{j} \bmod \sigma(j)\right)_{j \geq 0} \times\left(r_{i} \bmod p^{\pi(i)}\right)_{i \geq 1} \in \prod_{j \geq 0} \mathbb{Z} / \sigma(j) \mathbb{Z} \times \prod_{i \geq 1} \mathbb{Z} / p^{\pi(i)} \mathbb{Z} .
$$

Den Begriff der Signatur sollte man sich durch eine passende Darstellung der Milnorschen Basiselemente veranschaulichen. Dazu stelle man ein $Q(\varepsilon) P(R)$ in einem zu Abbildung 2.3 analogen Bild dar: $\varepsilon_{j}$ werde ins $\sigma$-Kästchen zum Index $j \geq 0$ geschrieben. Ins $\pi$-Kästchen in der $i$ ten Spalte und $k$ ten Zeile - wobei die Nummerierung der Zeilen mit $k=0$ beginne - schreibe man $r_{i, k}$, wenn $r_{i}$ die $p$-adische Darstellung $r_{i}=\sum_{k>0} r_{i, k} p^{k}$ mit $0 \leq r_{i, k}<p$ hat. Wir nennen dies die Ziffern-Darstellung von $Q(\varepsilon) P(R)$. Für $p=5$ und $Q(1,0,1,1) P(33,16,0,9)$ sieht diese zum Beispiel so aus:

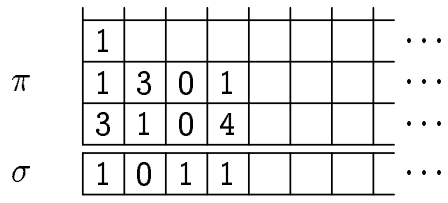

In den leeren Feldern stehe dabei Null. Ist $B$ wie in Abbildung 2.3 durch ein Muster aus schwarzen und weißen Kästchen gegeben, so erhält man die $B$-Signatur von $Q(\varepsilon) P(R)$ einfach durch Vergessen der Einträge in den nicht-geschwärzten Kästchen.

Eine endliche zulässige Hopf-Unteralgebra läßt sich schrittweise aufbauen, indem man die schwarzen Kästchen geschickt nummeriert:

Definition 2.2.7. 1) Seien $B=B(\Pi)$ und $B^{\prime}=B\left(\Pi^{\prime}\right)$ zulässige Hopf-Unteralgebren mit $B \subset B^{\prime}$. $B$ heißt Vorgänger von $B^{\prime}$, falls entweder

a) ein $i_{0} \geq 1$ existiert, sodaß

$$
\begin{aligned}
& \sigma(j)=\sigma^{\prime}(j) \quad \text { für alle } j, \\
& \pi(i)= \begin{cases}\pi^{\prime}(i) & i \neq i_{0}, \\
\pi^{\prime}\left(i_{0}\right)-1 & i=i_{0} .\end{cases}
\end{aligned}
$$

$B$ heißt dann genauer $\pi$-Vorgänger an der Stelle $i_{0}$ von $B^{\prime}$.

b) ein $j_{0} \geq 0$ existiert, sodaß

$$
\begin{aligned}
& \sigma(j)= \begin{cases}\sigma^{\prime}(j) & j \neq j_{0}, \\
\sigma^{\prime}\left(j_{0}\right)-1 & j=j_{0},\end{cases} \\
& \pi(i)=\pi^{\prime}(i) \quad \text { für alle } i .
\end{aligned}
$$

$B$ heißt dann genauer $\sigma$-Vorgänger an der Stelle $j_{0}$ von $B^{\prime}$.

$B^{\prime}$ nennen wir entsprechend Nachfolger von $B$.

2) Eine Filtrierung

$$
0=B\left(\Pi_{0}\right) \subset B\left(\Pi_{1}\right) \subset B\left(\Pi_{2}\right) \subset \cdots \subset B\left(\Pi_{n}\right)=B
$$

mit zulässigen $B\left(\Pi_{k}\right)$ heißt Schrittzerlegung von $B$, wenn $B\left(\Pi_{k}\right)$ für jedes $k$ ein Vorgänger von $B\left(\Pi_{k+1}\right)$ ist. 

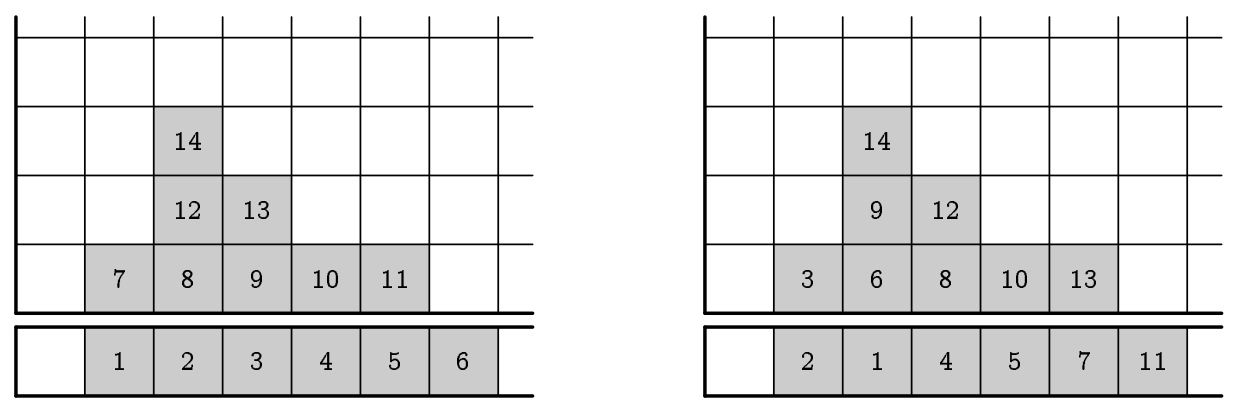

Abbildung 2.4: Zwei unterschiedliche Schrittzerlegungen derselben Hopf-Unteralgebra. In der Filtrierung $0=B\left(\Pi_{0}\right) \subset \cdots \subset B\left(\Pi_{14}\right)=B$ sei $B\left(\Pi_{k}\right)$ die Hopf-Unteralgebra, deren Profil aus den Kästchen mit einer Nummer $\leq k$ besteht.

Ist $B^{\prime}$ ein Nachfolger von $B$, so gibt es in der graphischen Darstellung gemäß Abbildung 2.3 genau ein Kästchen, das zu $B^{\prime}$, aber nicht zu $B$, gehört. Dieses nennen wir das $B^{\prime} \backslash B$-Kästchen.

Lemma 2.2.8. Jedes endliche zulässige B hat eine Schrittzerlegung.

Beweis. Es reicht zu zeigen, daß jedes endliche zulässige $B=B(\Pi) \neq 0$ einen Vorgänger $B\left(\Pi^{\prime}\right) \subset B$ hat. Angenommen es ist $\pi \not \equiv 0$. Wähle dann ein $i_{0} \geq 1$ mit $\pi\left(i_{0}\right)$ maximal, und definiere $\Pi^{\prime}$ durch

$$
\sigma^{\prime}(j)=\sigma(j) \quad \text { und } \quad \pi^{\prime}(i)= \begin{cases}\pi\left(i_{0}\right)-1 & i=i_{0} \\ \pi(i) & i \neq i_{0}\end{cases}
$$

Wir müssen zeigen, daß $\Pi^{\prime}$ wieder zulässig ist: da die $\sigma$-Komponente unverändert geblieben ist, gilt weiterhin 2.12, denn die $\pi$-Komponente ist ja nirgends größer geworden. Angenommen 2.11 wäre verletzt. Dann gäbe es $i, j \geq 1$ mit $\pi^{\prime}(i)>$ $j+\pi^{\prime}(i+j)$. Weil nach Voraussetzung $\pi(i) \leq j+\pi(i+j)$ muß $i+j=i_{0}$ sein, denn nur $\pi^{\prime}\left(i_{0}\right)$ ist kleiner als das entsprechende $\pi\left(i_{0}\right)$. Also ist $\pi^{\prime}(i)=\pi(i) \leq$ $\pi\left(i_{0}\right)=\pi^{\prime}\left(i_{0}\right)+1$, denn $i_{0}$ war so gewählt, daß $\pi\left(i_{0}\right) \geq \pi(i)$ für alle $i$. Da $j \geq 1$ gilt $\pi^{\prime}(i) \leq 1+\pi^{\prime}\left(i_{0}\right) \leq j+\pi^{\prime}(i+j)$ also doch.

Falls $\pi \equiv 0$, so wähle man $j_{0} \geq 0$ mit $\sigma\left(j_{0}\right)=2$ und setze

$$
\pi^{\prime}(i)=\pi(i) \quad \text { und } \quad \sigma^{\prime}(j)= \begin{cases}1 & j=j_{0}, \\ \sigma(j) & j \neq j_{0} .\end{cases}
$$

Auch dieses $\Pi^{\prime}$ ist zulässig: da $\pi^{\prime} \equiv 0$, sind 2.11 und 2.12 leere Bedingungen.

Abbildung 2.4 zeigt zwei unterschiedliche Schrittzerlegungen für das $B$ aus Abbildung 2.3. Dort sieht man auch, wie die Schrittzerlegung mit einer Nummerierung der schwarzen Kästchen zusammenhängt. Man kann diese Nummerierung dazu benutzen, eine Ordnung auf den möglichen $B$-Signaturen zu erklären: es gelte $\operatorname{sig}_{B}(x)<\operatorname{sig}_{B}(y)$, falls für ein geeignetes $m$ die ersten $m-1$ Ziffern bei $x$ und $y$ übereinstimmen, die $m$ te Ziffer von $x$ aber kleiner als die entsprechende Ziffer von $y$ ist. Es gibt eine kleinste Signatur, die identisch Null ist, und eine größte, bei der jede $\sigma$-Ziffer 1 und jede $\pi$-Ziffer $p-1$ ist. 
Lemma 2.2.9. $T_{B}=Q(\tilde{\varepsilon}) P(\tilde{R})$ hat maximale B-Signatur.

Beweis. Daß alle $\sigma$-Ziffern Eins sind ist nach Definition klar. Für die $\pi$-Ziffern bedenke man, daß $\tilde{r}_{i}=p^{\pi(i)}-1$ die $p$-adische Darstellung

$$
p^{\pi(i)}-1=(p-1)+(p-1) p+(p-1) p^{2}+\cdots+(p-1) p^{\pi(i)-1}
$$

hat. Alle $\pi$-Ziffern sind damit $p-1$, also maximal.

Wir zeigen jetzt, wie man den Übergang von $B$ zu einem Nachfolger $B^{\prime}$ mit der Theorie aus Abschnitt 2.1.2 in Beziehung setzen kann.

Definition 2.2.10. Sei $B^{\prime}=B\left(\Pi^{\prime}\right)$ ein Nachfolger von $B=B(\Pi)$. Definiere $Q=$ $Q_{B, B^{\prime}} \in A$ und $l=l_{B, B^{\prime}} \in \mathbb{N}$ wie folgt:

1) Falls $B^{\prime} \pi$-Nachfolger an der Stelle $i_{0}$ ist, sei $Q=P\left(0, \ldots, 0, p^{\pi\left(i_{0}\right)}\right)\left(\right.$ mit $i_{0}-1$ Nullen) und $l=p$.

2) Falls $B^{\prime} \sigma$-Nachfolger an der Stelle $j_{0}$ ist, sei $Q=Q_{j_{0}}$ und $l=2$.

Hat man eine Schrittzerlegung wie in 2.14, so sei $B_{m}=B\left(\Pi_{m}\right)$ und $l_{m}=l_{B_{m}, B_{m+1}}$. Wir schreiben auch $Q_{m}=Q_{B_{m}, B_{m+1}}$, falls die Gefahr der Verwechslung mit dem gleichbezeichneten Milnorschen Bocksteinoperator nicht besteht. $Q_{m}$ hat die Zifferndarstellung, bei der die $(m+1)$ te Ziffer Eins und alle anderen Ziffern Null sind. $l_{m}$ ist die Anzahl der möglichen Ziffern im $(m+1)$ ten Kästchen.

Das folgende Lemma zeigt, wie sich manche Multiplikationen in der Milnor-Basis vereinfachen, wenn ein Faktor maximale $B$-Signatur hat.

Notation. $a \doteq b$ soll bedeuten, daß $a=\lambda b$ mit $\lambda \in \mathbb{F}_{p}^{*}$ gilt.

Lemma 2.2.11. Sei $B$ endlich und zulässig, $Q(\varepsilon) P(R)$ beliebig.

1) Es ist

$$
T_{B} \cdot Q(\varepsilon) P(R)= \begin{cases} \pm Q(\varepsilon+\tilde{\varepsilon}) P(R+\tilde{R}) & \text { falls } \operatorname{sig}_{B}(Q(\varepsilon) P(R)) \equiv 0 \\ 0 & \text { sonst. }\end{cases}
$$

2) Sei $B^{\prime} \pi$-Nachfolger von $B$ an der Stelle $i_{0}, Q=Q_{B, B^{\prime}}$ und $0 \leq k<l$. $\operatorname{sig}_{B}(Q(\varepsilon) P(R))$ sei maximal. $z \in\{0, \ldots, p-1\}$ sei die $B^{\prime} \backslash B$-Ziffer von $Q(\varepsilon) P(R)$. Dann ist

$$
Q^{k} \cdot Q(\varepsilon) P(R) \doteq \begin{cases}Q(\varepsilon) P\left(\tilde{R}+\left(0, \ldots, 0, k p^{\pi\left(i_{0}\right)}\right)\right) & \text { falls } z+k<p \\ 0 & \text { sonst. }\end{cases}
$$

3) Sei $B^{\prime} \sigma$-Nachfolger von $B$ an der Stelle $j_{0}, Q=Q_{B, B^{\prime}} . z=\varepsilon_{j_{0}}$ sei die $B^{\prime} \backslash B$ Ziffer von $Q(\varepsilon) P(R)$. Dann gilt

$$
Q \cdot Q(\varepsilon) P(R)= \begin{cases} \pm Q(\varepsilon+(0, \ldots, 0,1)) P(R) & \text { falls } z=0 \\ 0 & \text { sonst. }\end{cases}
$$


Beweis von 1). Sei $T_{B}=Q(\tilde{\varepsilon}) P(\tilde{R})$. Wir müssen die Multiplikation $Q(\tilde{\varepsilon}) P(\tilde{R}) Q(\varepsilon) P($ $R)$ betrachten. Dazu greifen wir uns zunächst den mittleren Teil $P(\tilde{R}) Q(\varepsilon)$ heraus. Nach 1.1.14 gilt

$$
P(\tilde{R}) Q_{j}=Q_{j} P(\tilde{R})+\sum_{t>0} Q_{j+t} P\left(\tilde{r}_{1}, \ldots, \tilde{r}_{t-1}, \tilde{r}_{t}-p^{j}, \tilde{r}_{t+1}, \ldots\right) .
$$

Wir dürfen die Summe auf der rechten Seite ignorieren: der Term $Q_{j+t} \cdot P(\cdots)$ taucht dort nur auf, wenn $\tilde{r}_{t}=p^{\pi(i)}-1 \geq p^{j}$ ist. Dann ist $\pi(i)>j$, sodaß nach 2.12 $\sigma(t+j)=2$. Wegen $\sigma(t+j)=2$ ist dann aber $Q_{j+t}$ ein Faktor von $Q(\tilde{\varepsilon})$, sodaß der Summand bei der Multiplikation mit $Q(\tilde{\varepsilon})$ wegen $Q_{j+t}^{2}=0$ wegfällt.

Wir dürfen also von $Q(\tilde{\varepsilon}) P(\tilde{R}) Q(\varepsilon) P(R)$ zu $Q(\tilde{\varepsilon}) Q(\varepsilon) P(\tilde{R}) P(R)$ übergehen. Falls $\varepsilon_{j} \not \equiv 0 \bmod \sigma(j)$ für ein $j$, so ist $\varepsilon_{j}=1$ und $\sigma(j)=2$, sodaß $Q_{j}$ sowohl ein Faktor von $Q(\varepsilon)$, wie auch von $Q(\tilde{\varepsilon})$ ist. Wegen $Q_{j}^{2}=0$ wäre also das Produkt null, wie behauptet.

Wir nehmen also jetzt $\varepsilon_{j} \equiv 0 \bmod \sigma(j)$ an. $Q(\tilde{\varepsilon})$ und $Q(\varepsilon)$ ergeben zusammen das behauptete $\pm Q(\varepsilon+\tilde{\varepsilon})$, sodaß wir uns nur noch auf die Multiplikation $P(\tilde{R}) \cdot P(R)$ zu konzentrieren brauchen. Wir benutzen jetzt Milnors Multiplikationsalgorithmus, wie in Abbildung 1.1 beschrieben. Sei $X=\left(x_{i, j}\right)_{i, j>0, i+j \neq 0}$ eine Matrix wie in Lemma 1.1.11. Wir behaupten, daß nur die triviale Matrix mit $x_{k, 0}=\tilde{r}_{k}, x_{0, k}=r_{k}$ und $x_{k, i}=0$ für $k, i>0$ zum Produkt beiträgt.

Sei also $x_{k, i} \neq 0$ für gewisse $k, i>0$. Wähle ein solches $(k, i)$ mit maximalem $k$. Wegen

$$
\tilde{r}_{k}=x_{k, 0}+p x_{k, 1}+p^{2} x_{k, 2}+\cdots
$$

ist $\tilde{r}_{k} \geq p^{i} x_{k, i}$, also $1 \leq x_{k, i}<p^{\pi(k)-i}$. Aus 2.11 folgt $\pi(k)-i \leq \pi(k+i)$, sodaß $x_{k, i} \not \equiv 0 \bmod p^{\pi(k+i)}$.

Andererseits ist $x_{k+i, 0} \equiv-1 \bmod p^{\pi(k+i)}$; da $k$ maximal gewählt wurde, ist ja $x_{k+i, j}=0$ für $j>0$, sodaß $x_{k+i, 0}=\tilde{r}_{k+i}=p^{\pi(k+i)}-1$. Also verschwindet nach Korollar 1.1.13 der Multinomialkoeffizient $\left(x_{k+i, 0}|\cdots| x_{k, i}|\cdots| x_{0, k+i}\right)$ modulo $p$, da bei der Addition $x_{k, i}+x_{k+i, 0}$ zur Basis $p$ Überträge auftreten. Da dieser Multinomialkoeffizient ein Faktor von $\beta(X)$ ist, trägt die Matrix $X$ nichts zum Produkt $P(\tilde{R}) \cdot P(R)$ bei.

Es bleibt also nur die Matrix mit $x_{k, 0}=\tilde{r}_{k}, x_{0, k}=r_{k}$ und $x_{i, j}=0$ für $i, j>0$ übrig. Deren Beitrag zum Produkt ist

$$
\left(\tilde{r}_{1} \mid r_{1}\right) \cdots\left(\tilde{r}_{n} \mid r_{n}\right) \cdot P(R+\tilde{R})
$$

Die Binomialkoeffizienten haben alle die Form $\left(p^{\pi(k)}-1 \mid r_{k}\right)$. Wiederum nach Korollar 1.1.13 verschwindet dieser Faktor, falls $r_{k} \not \equiv 0 \bmod p^{\pi(k)}$ ist. Im anderen Fall erhält man mit Lemma 1.1.12, daß $\left(p^{N}-1 \mid p^{N} \cdot K\right) \equiv 1 \bmod p$ für beliebige $N$ und $K$, was wir hier für $N=\pi(k)$ und $K=r_{k} / p^{\pi(k)}$ benutzen können; die Darstellung zur Basis $p$ ist nämlich

$$
\begin{aligned}
& p^{N}-1=(p-1)+(p-1) p+(p-1) p^{2}+\cdots+(p-1) p^{N-1} \\
& p^{N} \cdot K=0+\quad 0 \cdot p+\quad 0 \cdot p^{2}+\cdots+\quad 0 \cdot p^{N-1}+\sum_{i \geq N} k_{i} p^{i}
\end{aligned}
$$


sodaß

$$
\begin{aligned}
\left(p^{N}-1 \mid p^{N} K\right) & \equiv(p-1 \mid 0) \cdots(p-1 \mid 0) \cdot \prod_{i \geq N}\left(0 \mid k_{i}\right) \bmod p \\
& \equiv 1 \bmod p
\end{aligned}
$$

Damit ist $P(\tilde{R}) P(R)=P(\tilde{R}+R)$, wie behauptet.

Wir geben dieses Resultat noch einmal mit anderen Worten wieder: Falls der zweite Faktor $Q(\varepsilon) P(R)$ die $B$-Signatur Null hat, so erzwingt die Linksmultiplikation mit $T_{B}$ einfach die maximale $B$-Signatur; die anderen Ziffern von $Q(\varepsilon) P(R)$ werden nicht verändert. Hat der zweite Faktor eine von Null verschiedene $B$-Signatur, so wird er annulliert. Insbesondere ist

$$
T_{B} A=\mathbb{F}_{p}\left\{Q(\varepsilon) P(R) \mid \operatorname{sig}_{B}(Q(\varepsilon) P(R)) \text { maximal }\right\} .
$$

Beweis von 2). Wie man sich leicht überlegt, reicht es die Aussage für $k=1 \mathrm{zu}$ beweisen, weil man $Q^{k}$ als $Q \cdots Q$ schreiben kann. Als erstes betrachten wir wieder $Q \cdot Q(\varepsilon)$. Zur Abkürzung sei $P_{N}=P(0, \ldots, 0, N)$, sodaß $Q=P_{p^{\pi\left(i_{0}\right)}}$. Aus Lemma 1.1.14 kennen wir die Kommutatorrelation

$$
P_{N} Q_{j}=Q_{j} P_{N}+ \begin{cases}Q_{i_{0}+j} P_{N-p^{j}} & \text { falls } N \geq p^{j} \\ 0 & \text { sonst. }\end{cases}
$$

Wir behaupten, daß wieder nur der erste Summand von Bedeutung ist. Wegen $N<$ $p^{\pi^{\prime}\left(i_{0}\right)}$ folgt aus 2.12 - angewendet auf $\left(\pi^{\prime}, \sigma^{\prime}\right)-\operatorname{da} \beta \sigma\left(i_{0}+j\right)=2$, sodaß $Q_{i_{0}+j}$ immer ein Bockstein aus $B$ ist. Sei $\left\{Q_{j_{1}}, \ldots, Q_{j_{L}}\right\}$ mit $j_{1}<\cdots<j_{L}$ die Menge dieser Bocksteins. Durch wiederholte Anwendung erhalten wir aus 2.16 zunächst

$P_{N} Q_{j_{1}} \cdots Q_{j_{L}}=Q_{j_{1}} \cdots Q_{j_{L}} P_{N}+\sum(L$-faches Bocksteinprodukt aus $B) \cdot($ irgendwas $)$.

Unter den Faktoren auf der rechten Seite kann der kleinste Bockstein aus $B$ nicht auftreten, weil das $Q_{i_{0}+j}$ aus 2.16 wegen $i_{0} \geq 1$ niemals $Q_{j_{1}}$ ist. Also muß dort jeweils ein Bockstein doppelt vorkommen und annulliert damit den Summanden.

Schreibt man $Q(\varepsilon)=Q_{j_{1}} \cdots Q_{j_{L}} \cdot Q_{j_{L+1}} \cdots Q_{j_{L^{\prime}}}=Q(\tilde{\varepsilon}) \cdot Q_{j_{L+1}} \cdots Q_{j_{L^{\prime}}}$, so ist $P Q(\varepsilon)=Q(\tilde{\varepsilon}) \cdot P Q_{j_{L+1}} \cdots Q_{j_{L^{\prime}}}$. Wendet man nun 2.16 auf den zweiten Teil an, so kann man wiederum die Störterme ignorieren: es wurde schon festgestellt, daß dabei nur Bocksteinoperatoren aus $B$ auftreten, und diese werden durch den Vorfaktor $Q(\tilde{\varepsilon})$ zu Null.

Also ist $Q \cdot Q(\varepsilon)=Q(\varepsilon) \cdot Q$ und wir müssen nur noch $Q \cdot P(R)$ betrachten. Sei $X=\left(x_{i, j}\right)$ eine Multiplikationsmatrix. Wir behaupten, daß wieder nur die triviale Matrix mit $x_{i, j}=0$ für $i \geq 1$ eine Rolle spielt. Für dieses $X$ gilt offenbar $x_{i_{0}, 0}=p^{\pi\left(i_{0}\right)}$ und $x_{i, 0}=0$ für $i \neq i_{0}$. Ihr Beitrag zum Produkt ist deswegen $\left(p^{\pi\left(i_{0}\right)} \mid r_{i_{0}}\right) \cdot P(R+$ $\left.\left(0, \ldots, 0, p^{\pi\left(i_{0}\right)}\right)\right)$. Nach Lemma 1.1 .12 ist der Binomialkoeffizient gerade $(1 \mid z)=$ $z+1$, was mit der Behauptung übereinstimmt.

Sei also $X$ jetzt eine andere Matrix, dh. es sei $x_{i, j} \neq 0$ für gewisse $i, j \geq 1$. Offenbar ist $i=i_{0}$, da in $Q$ nur der $i_{0}$ te Eintrag von Null verschieden ist. Sei 
$j_{0}$ das größte $j$ mit $x_{i_{0}, j} \neq 0$. Dieselbe Abschätzung wie oben zeigt, daß $x_{i_{0}, j_{0}} \not \equiv$ $0 \bmod p^{\pi\left(i_{0}+j_{0}\right)}$ gilt. Die Maximalität von $j$ impliziert $x_{i, i_{0}+j_{0}}=0$ für alle $i \geq 1$. Also hat man $x_{0, i_{0}+j_{0}}=r_{i_{0}+j_{0}}$. Dies ist $\equiv-1 \bmod p^{\pi\left(i_{0}+j_{0}\right)}$ weil $Q(\varepsilon) P(R)$ maximale $B$-Signatur hat. Deswegen tritt bei der Addition $x_{i_{0}, j_{0}}+x_{0, i_{0}+j_{0}}$ ein Übertrag auf und es ist $\beta(X)=0$.

Beweis von 3). Dies ist völlig trivial: $z$ ist eben genau dann Null, wenn der Bockstein $Q$ noch nicht in $Q(\varepsilon)$ auftaucht.

2) und 3) lassen sich so zusammenfassen: wenn $B^{\prime}$ ein Nachfolger von $B$ ist, so enthält das Profil von $B^{\prime}$ genau ein Kästchen mehr als $B$. Hat $Q(\varepsilon) P(R)$ bereits volle $B$-Signatur, so erhöht die Linksmultiplikation mit $Q=Q_{B, B^{\prime}}$ einfach die Ziffer in diesem zusätzlichen Kästchen, solange dabei kein Übertrag entsteht. Entsteht ein Übertrag, so ist das Ergebnis Null. Insbesondere ergibt sich

$$
Q_{B, B^{\prime}}^{k} T_{B} A=\mathbb{F}_{p}\left\{Q(\varepsilon) P(R) \mid \operatorname{sig}_{B}(Q(\varepsilon) P(R)) \text { maximal, } B^{\prime} \backslash B \text {-Ziffer } \geq k\right\} .
$$

Den Kontakt zu Abschnitt 2.1.2 stellt das folgende Korollar her.

Korollar 2.2.12. Sei B eine endliche zulässige Hopf-Unteralgebra und B' ein Nachfolger von $B . Q$ und $l$ seien wie oben definiert. Dann gilt: Linksmultiplikation mit $Q$ definiert eine Abbildung

$$
Q: T_{B} A \rightarrow T_{B} A
$$

und diese induziert eine freie $\mathbb{F}_{p}[Q] /\left(Q^{l}\right)$-Modulstruktur auf $T_{B} A$ mit $Q^{l-1} T_{B} A=$ $T_{B^{\prime}} A$.

Beweis. Aus 2.2.11,1) und 2) folgt $Q T_{B}=T_{B} Q$. Deswegen ist $Q T_{B} A=T_{B} Q A \subset$ $T_{B} A$, sodaß $Q: T_{B} A \rightarrow T_{B} A$ wohldefiniert ist. 2.2.11, 2) und 3) implizieren $Q^{l-1} T_{B} A$ $=T_{B^{\prime}} A$ und $Q^{l} T_{B} A=0 . T_{B} A$ ist als $\mathbb{F}_{p}[Q] /\left(Q^{l}\right)$-Modul frei weil

$$
\left\{Q(\varepsilon) P(R) \mid B \text {-Signatur maximal und } B^{\prime} \backslash B \text {-Ziffer }=0\right\}
$$

offenbar eine $\mathbb{F}_{p}[Q] /\left(Q^{l}\right)$-Basis ist.

Man beachte, daß $Q^{k} T_{B} A$ eine Vektorraumbasis hat, die eine Teilmenge der Milnor-Basis ist. Dies ist für die Implementierung auf dem Computer von großer Bedeutung: weder bei der Inklusion $T_{B} A \rightarrow A$, noch bei der Projektion $T_{B}: A \rightarrow T_{B} A$ muß nennenswert gerechnet werden, und dasselbe gilt auch für die Operation von $Q$.

Wir bemerken noch, daß sich 2.2.12 offenbar auf Auflösungen überträgt:

Korollar 2.2.13. Sei $C_{*}$ eine partielle Auflösung. Dann ist $T_{B} C_{*}$ ein freier $\mathbb{F}_{p}[Q] /($ $\left.Q^{l}\right)$-Modul mit $Q^{l-1} T_{B} C_{*}=T_{B^{\prime}} C_{*}$.

Beweis. Klar, weil $C_{*}$ ein freier $A$-Modul ist.

Zum Abschluß noch eine Bemerkung zur Demystifikation, im Anschluß an 2.2.3:

Bemerkung 2.2.14. Als $A$-Rechtsmodul kann $T_{B} A$ mit dem Hopf-Quotienten $B \backslash \backslash A$ $=\mathbb{F}_{p} \otimes_{B} A$ identifiziert werden. Es ist ja $T_{B} A=T_{B} B \otimes_{B} A$, sodaß nur $T_{B} B \cong_{B} \mathbb{F}_{p}$ gezeigt werden muß. Dies ist aber klar, denn nach Lemma 2.2.11 ist $T_{B} B=\mathbb{F}_{p}\left\{T_{B}\right\}$. 


\subsubsection{Von Unten, allgemein}

Wir kommen nun zu unserem Algorithmus. Wir wollen zeigen, wie man im Bereich $T-\tau_{B}>S \cdot d_{B}$ mithilfe von Lemma 2.1.9 die Berechnung von $C_{*}$ auf das Rechnen mit $T_{B} C_{*}$ zurückführen kann.

Seien $S, T \in \mathbb{N}$ und $C_{*}$ eine in $(S, T)$ erweiterbare Auflösung. Sei $B \subset A$ eine endliche zulässige Hopf-Unteralgebra mit einer Schrittzerlegung

$$
0=B\left(\Pi_{0}\right) \subset B\left(\Pi_{1}\right) \subset \cdots \subset B\left(\Pi_{N}\right)=B .
$$

Zur Abkürzung schreiben wir $B_{m}=B\left(\Pi_{m}\right), T_{m}=T_{B_{m}}, \tau_{m}=\left|T_{m}\right|, Q_{m}=Q_{B_{m}, B_{m+1}}$ und $l_{m}=l_{B_{m}, B_{m+1}}$. Das Ziel ist, die Erweiterung von $C_{*} \operatorname{im} \operatorname{Grad}(S, T)$ mithilfe von Lemma 2.1.2 auf Rechnungen in $T_{N} C_{*}$ zurückzuführen. Dabei folgt man der Zerlegung durch die $B_{m}$, indem man die Rechnung in $T_{m} C_{*}$ auf $l_{m}$ Rechnungen mit $T_{m+1} C_{*}$ reduziert. Der Schritt von $T_{m} C_{*}$ zu $T_{m+1} C_{*}$ benutzt dabei die kurzen exakten Sequenzen

$$
\begin{gathered}
T_{m+1} C_{*}=Q_{m}^{l_{m}-1} T_{m} C_{*} \longmapsto T_{m} C_{*} \stackrel{Q_{m}}{\longrightarrow} Q_{m} T_{m} C_{*}, \\
T_{m+1} C_{*}=Q_{m}^{l_{m}-1} T_{m} C_{*} \longrightarrow Q_{m} T_{m} C_{*} \stackrel{Q_{m}}{\longrightarrow} Q_{m}^{2} T_{m} C_{*}, \\
\vdots \\
T_{m+1} C_{*}=Q_{m}^{l_{m}-1} T_{m} C_{*} \longrightarrow Q_{m}^{l_{m}-2} T_{m} C_{*} \stackrel{Q_{m}}{\longrightarrow} Q_{m}^{l_{m}-1} T_{m} C_{*} .
\end{gathered}
$$

Daß dies funktioniert sagt

Satz 2.2.15. Falls $T-\tau_{B}>S \cdot d_{B}$, so sind alle auftretenden Sequenzen 2.18, . ,2.20 in den nötigen Bigraden freundlich.

Vor dem Beweis brauchen wir noch zwei Lemmas.

Lemma 2.2.16. Für alle $m$ ist $\left(l_{m}-1\right)\left|Q_{m}\right| \leq d_{B}$.

Beweis. So war $d_{B}$ gerade definiert.

Lemma 2.2.17. $C_{*}$ sei in $(S, T)$ erweiterbar. Dann ist für $0 \leq m<n$ und $0 \leq k<$ $l_{m}$

$$
H_{s, t}\left(Q_{m}^{k} T_{m} C_{*}\right)=0 \quad \text { für } \quad(s, t) \in R_{S, T}^{o} \cap A^{d_{B}, 0-} .
$$

Beweis. Für $k=m=0$ ist dies wahr, weil $C_{*}$ nach Voraussetzung in $R_{S, T}^{o} \backslash\{0\}$ exakt ist. Sei die Aussage für $k=0$ und $m=m_{0}<N$ schon bekannt. Wegen Lemma 2.1.9 gilt sie dann auch für die anderen $k<l_{m}$ : Dazu setzen wir $M_{*}=T_{m} C_{*}, Q=Q_{m}$, $l=l_{m}, \rho=d_{B}$ und $r=0$. Wie gerade gesehen ist dann $(l-1) q \leq \rho$, sodaß also $l q / 2 \leq \rho$ und $r^{\prime}=0$, letzteres weil $(l-k) q-\rho \leq(l-1) q-\rho \leq 0$. Also ist Lemma 2.1.9 anwendbar und die Behauptung für $k<l$ gezeigt. Da $Q_{m}^{l_{m}-1} T_{m} C_{*}=T_{m+1} C_{*}$ ist, haben wir die Behauptung damit auch für $k=0$ und $m=m_{0}+1$ gezeigt, was die Induktion schließt.

Der Satz ist jetzt eine einfache Konsequenz: 


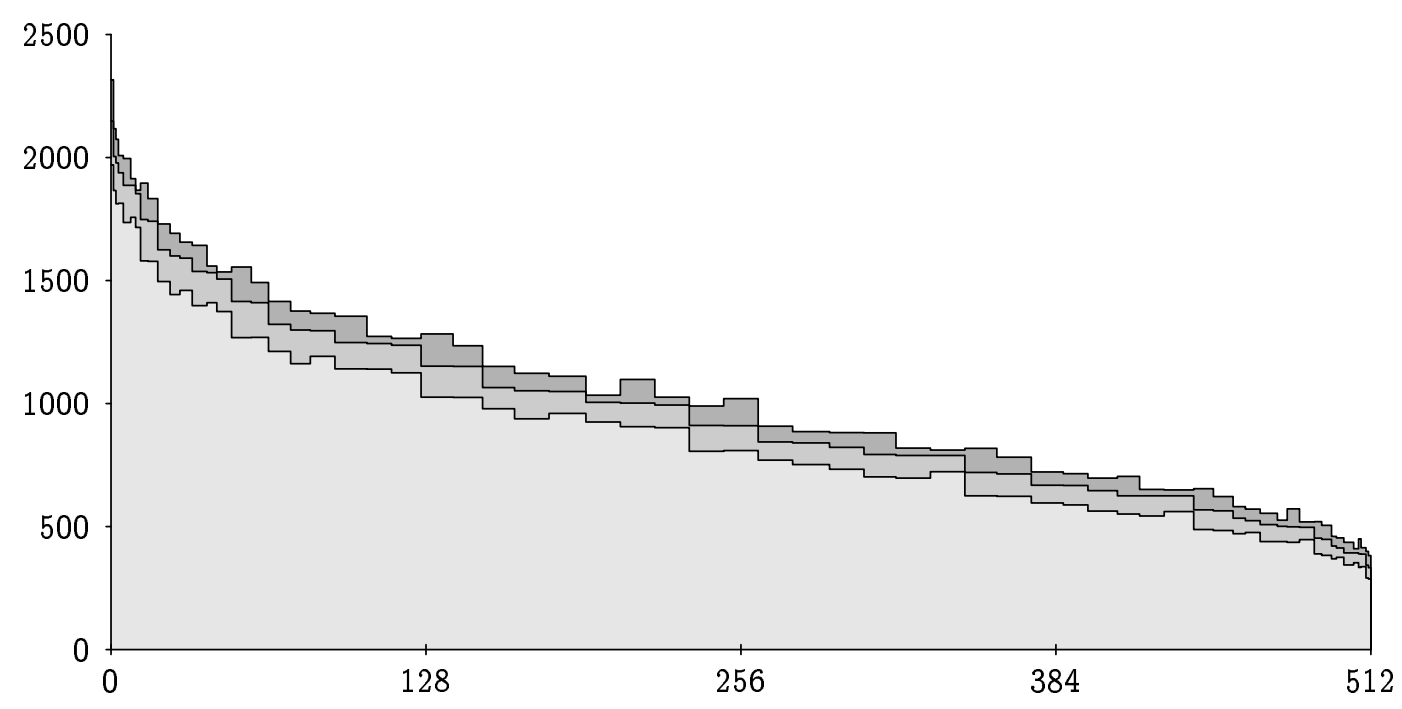

Abbildung 2.5: Zerlegung der Matrizen bei der Berechnung der Homologie von $C_{11,220} \rightarrow C_{10,220} \rightarrow C_{9,220}$ für $p=2$. Diese Vektorräume haben die Dimension 439547, 488273 bzw. 521837. Benutzt wurde die Unteralgebra $B$ mit Profil $\pi=(3,3,2,1)$; für $B$ hat $\operatorname{man} d_{B}=15, \tau_{B}=64$, sodaß Satz 2.2 .15 für $(S, T)=(10,220)$ anwendbar ist. Nach Anwendung der Lemmas 2.1.2 und 2.1.3 bleiben eine Homologieberechnung und 511 Hebungsprobleme übrig. Gezeigt werden die Dimensionen der dabei auftretenden Vektorräume $T_{B} C_{*, 210-}$ ? mit $* \in\{9,10,11\}$. Der obere Bereich bezieht sich auf $C_{9}$, der mittlere auf $C_{10}$ und der untere auf $C_{11}$.

Beweis des Satzes. Wir müssen zeigen, daß für $T-\tau_{B}>S \cdot d_{B}$ alle auftretende Bigrade $(s, t)$ in $A^{d_{B}, 0-}$ liegen. Man bedenke, daß die Inklusion $T_{m+1} C_{*} \longmapsto$ $Q_{m}^{k} T_{m} C_{*}$ den internen Grad um $\left(l_{m}-1-k\right) q_{m}$ erhöht, wobei $q_{m}=\left|Q_{m}\right|$. Wird Lemma 2.1.2 also auf 2.18 angewendet, so ersetzt es die Berechnung der Homologie $H_{s, t}\left(T_{m} C_{*}\right)$ durch die Berechnung der Homologie $H_{s, t}\left(Q T_{m} C_{*}\right)$ und die Berechnung eines Lifts $L_{s, t-\left(l_{m}-1\right) q_{m}}\left(T_{m+1} C_{*}\right)$. Durch wiederholte Anwendung auf $2.18, \ldots, 2.20$ wird $H_{s, t}\left(T_{m} C_{*}\right)$ schließlich durch

$$
H_{s, t}\left(T_{m+1} C_{*}\right) \text { und } L_{s, t-q_{m}}\left(T_{m+1} C_{*}\right), L_{s, t-2 q_{m}}\left(T_{m+1} C_{*}\right), \ldots, L_{s, t-\left(l_{m}-1\right) q_{m}}\left(T_{m+1} C_{*}\right)
$$

ersetzt. Dieselbe Verteilung ergibt sich bei Anwendung von Lemma 2.1.3 auf das Hebungsproblem $L_{s, t}\left(T_{m} C_{*}\right)$. Summiert man nun über alle $m$ zwischen 0 und $N-1$, so ergibt sich als maximale Verschiebung des internen Grads $\sum_{0<m<N}\left(l_{m}-1\right)\left|Q_{m}\right|$. Wie man sich leicht überlegt ist dies aber gerade $\tau_{B}$.

Bemerkung 2.2.18 (vgl. Bemerkung 2.2.3). Angenommen $C_{*}$ wäre eine vollständige $A$-Auflösung von $\mathbb{F}_{p}$. Nach Satz 2.2.1 kann man $C_{*}$ dann auch als $B$-Auflösung betrachten. Wie in 2.2 .14 bemerkt, ist $T_{B} B \cong \mathbb{F}_{p}$, sodaß $T_{B} C_{*}=\mathbb{F}_{p} \otimes_{B} C_{*}$. Für $k=0$ ist Lemma 2.2.17 dann einfach ein bekanntes Verschwindungsresultat für $H_{*, *}\left(T_{B} C_{*}\right)=\operatorname{Tor}_{*, *}^{B}\left(\mathbb{F}_{p}, \mathbb{F}_{p}\right)$ (siehe zB. [5] und/oder [33]).

Wir beschließen die Diskussion der unteren Varianten mit ein paar Statistiken. In Abbildung 2.5 sieht man wie Satz 2.2.15 bei der Berechnung von $\operatorname{Ext}_{A}^{11,220}\left(\mathbb{F}_{2}, \mathbb{F}_{2}\right)$ für 


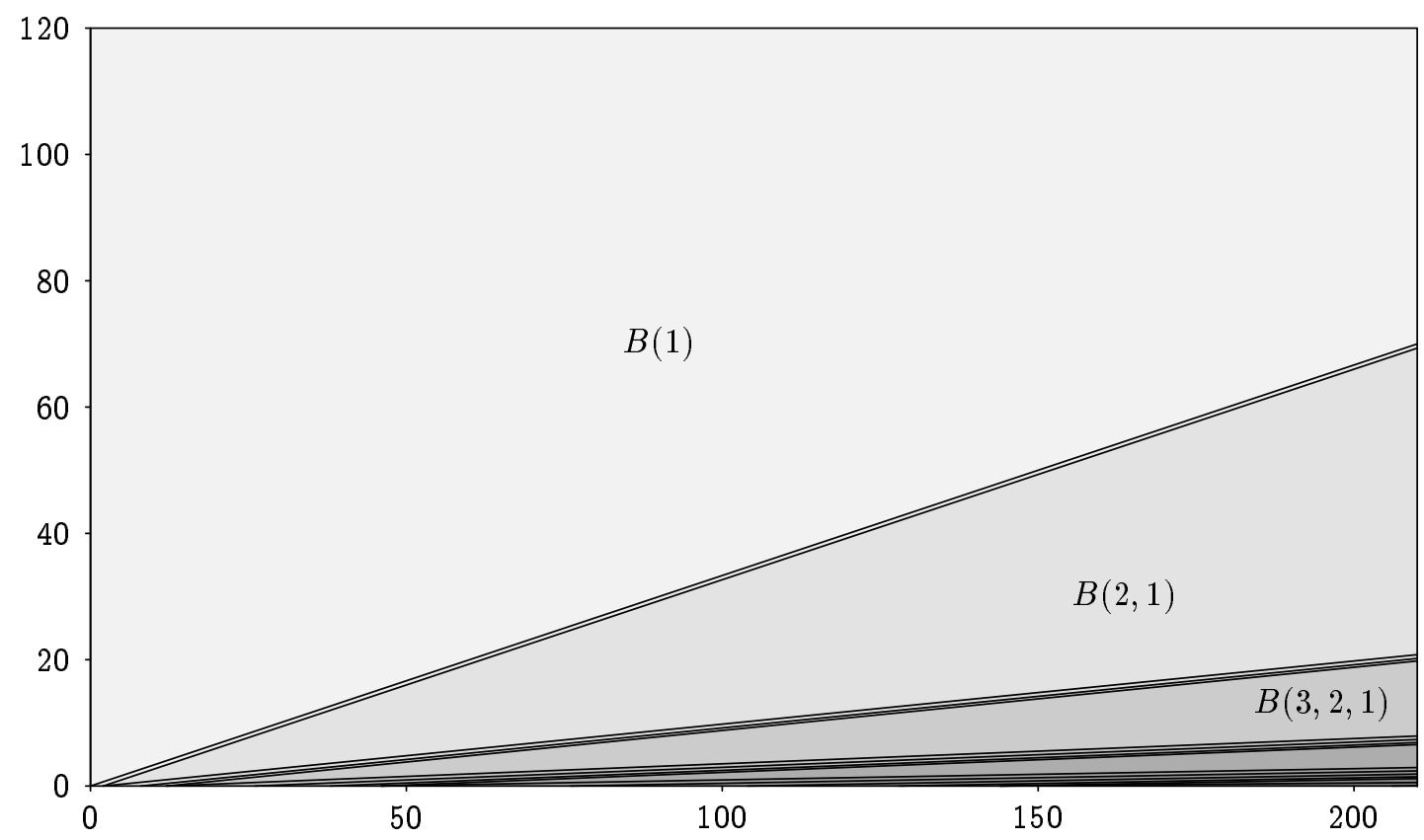

Abbildung 2.6: Anwendbarkeit von Satz 2.2.15 für $p=2$. Für die Folge der Hopf-Unteralgebren $B(1), B(1,1), B(2,1), B(2,1,1), B(2,2,1)$, usw. wird jeweils der Bereich $t-\tau_{B}>d_{B}(s-1)$ gezeigt. Das verwendete Koordinatensystem ist $(x, y)=(t-s, s)$.

$p=2$ angewendet wird. Die Methode ist hier sehr effektiv, weil man eine große Unteralgebra $B=B(3,3,2,1)$ verwenden kann; statt mit Vektorräumen der Dimension $\approx 500000$ rechnen zu müssen, kommt man mit solchen der Dimension $\leq 2500$ aus.

Allerdings ist die untere Variante weit weniger machtvoll, wenn $s$ im Vergleich zu $t-s$ groß wird. Dies sieht man in Abbildung 2.6, in der in $(s, t-s)$-Koordinaten jeweils die Bereiche $t-\tau_{B}>d_{B} s$ für gewisse $B$ gezeigt werden. In diesem problematischen Bereich läßt sich jedoch die obere Version des Lemmas einsetzen, was wir als nächstes erklären möchten.

\subsubsection{Von Oben}

Wir benutzen wieder die Zerlegung von $C_{*}$ durch die Sequenzen $2.18, \ldots, 2.20$, wollen aber jetzt Lemma 2.1.8 statt Lemma 2.1.9 anwenden.

Sei also wie oben $B \subset A$ eine endliche zulässige Hopf-Unteralgebra mit einer Schrittzerlegung $0=B_{0} \subset \cdots \subset B_{N}=B$. Wir setzen

$$
\rho=\rho_{B}=\min \left\{\left|Q_{B_{m}, B_{m+1}}\right|: m=0, \ldots, N-1\right\} .
$$

Unser Ziel ist

Satz 2.2.19. Ist $C_{*}$ in $(S, T)$ erweiterbar, so sind für $T<\rho_{B} \cdot S$ alle 2.18, . ,2.20 in allen nötigen Bigraden freundlich. 
Zum Beweis brauchen wir wieder zwei Lemmas:

Lemma 2.2.20. Für alle $Q_{m}$ ist $\left|Q_{m}\right| \geq \rho$.

Beweis. So ist $\rho_{B}$ gerade definiert.

Lemma 2.2.21. Ist $C_{*}$ in $(S, T)$ erweiterbar, so ist $H_{s, t}\left(T_{m}\right)=0$ für $(s, t) \in R_{S, T}^{o} \cap$ $A^{\rho,-\rho m+}$.

Beweis. Durch Induktion nach $m$ folgt dies aus Lemma 2.1.8. Der Induktionsanfang $m=0$ ist klar. Beim Schritt $m \Rightarrow m+1$ setzen wir $M=T_{m} C_{*}, Q=Q_{m}, l=l_{m}$, $r=-\rho m$ und $k=l-1$. Wegen $-2 \rho+(l-k) q=-2 \rho+q=-\rho+(q-\rho) \geq-\rho$ ist $r^{\prime}=r+\min \{-\rho,-2 \rho+(l-k) q\}=r-\rho=-m \rho-\rho=-(m+1) \rho$, wie behauptet.

Beweis des Satzes. Durch wiederholte Anwendung von Lemma 2.1 .2 auf $2.18, \ldots, 2.20$ wird das Homologieproblem zu $H_{s, t}\left(T_{m} C_{*}\right)$ auf folgende Art zerlegt:

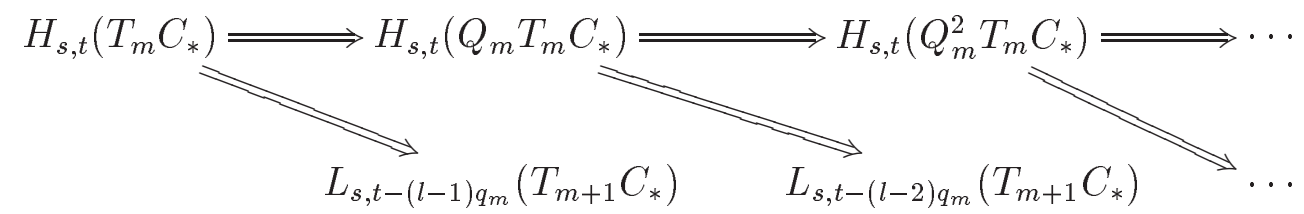

Dieselbe Verteilung erhält man auch bei Anwendung von Lemma 2.1.3 auf $L_{s, t}\left(T_{m} C_{*}\right)$. Damit Lemmas 2.1.2 und 2.1.3 anwendbar sind, muß $T_{m+1} C_{*}$ in den Bigraden der unteren Zeile, also in $\left(s, t-q_{m}\right), \ldots,\left(s, t-(l-1) q_{m}\right)$, exakt sein. Wir schauen uns also die auftretenden $t$-Komponenten genauer an: für $m=0$ braucht man die Exaktheit für $t \leq T-q_{0} \leq T-\rho$. Für $m=1$ braucht man $t \leq T-\rho-k q_{1} \leq T-\rho-k \rho \leq T-2 \rho$. Für ein allgmeines $m$ erhält man schließlich $t \leq T-(m+1) \rho$. Nach Lemma 2.2.21 ist $T_{m+1} C_{*}$ aber dort jeweils wie gewünscht exakt.

\subsubsection{Von Oben (de-Luxe-Variante)}

Die Theorie aus Abschnitt 2.2.2 kann man noch ein bißchen Verallgemeinern. Sei dazu $B(\pi, \sigma)$ eine zulässige Hopf-Unteralgebra, deren Profil kein Kästchen aus den ersten $i_{0}-1$ Spalten enthält. Es sei also $\sigma(i)=1$ für $i<i_{0}-1, \pi(i)=0$ für $i<i_{0}$. Zu $u$ mit $0 \leq u \leq \pi\left(i_{0}\right)$ bilden wir

$$
B^{u}=\left\{Q(\varepsilon) P(R) \in B \mid r_{i_{0}} \equiv 0 \bmod p^{u}\right\} .
$$

Offenbar gilt

$$
B^{\prime}=B^{\pi\left(i_{0}\right)} \subset B^{\pi\left(i_{0}\right)-1} \subset \cdots \subset B^{0}=B,
$$

wobei $B^{\prime}=B\left(\pi^{\prime}, \sigma^{\prime}\right)$ aus $B$ durch Streichen der $i_{0}$ ten Spalte entstanden ist:

$$
\begin{aligned}
\pi^{\prime}(i) & =\pi(i) \quad \text { für } i \neq i_{0} \\
\sigma^{\prime}(i) & =\sigma(i) \quad \text { für alle } i \\
\pi^{\prime}\left(i_{0}\right) & =0
\end{aligned}
$$




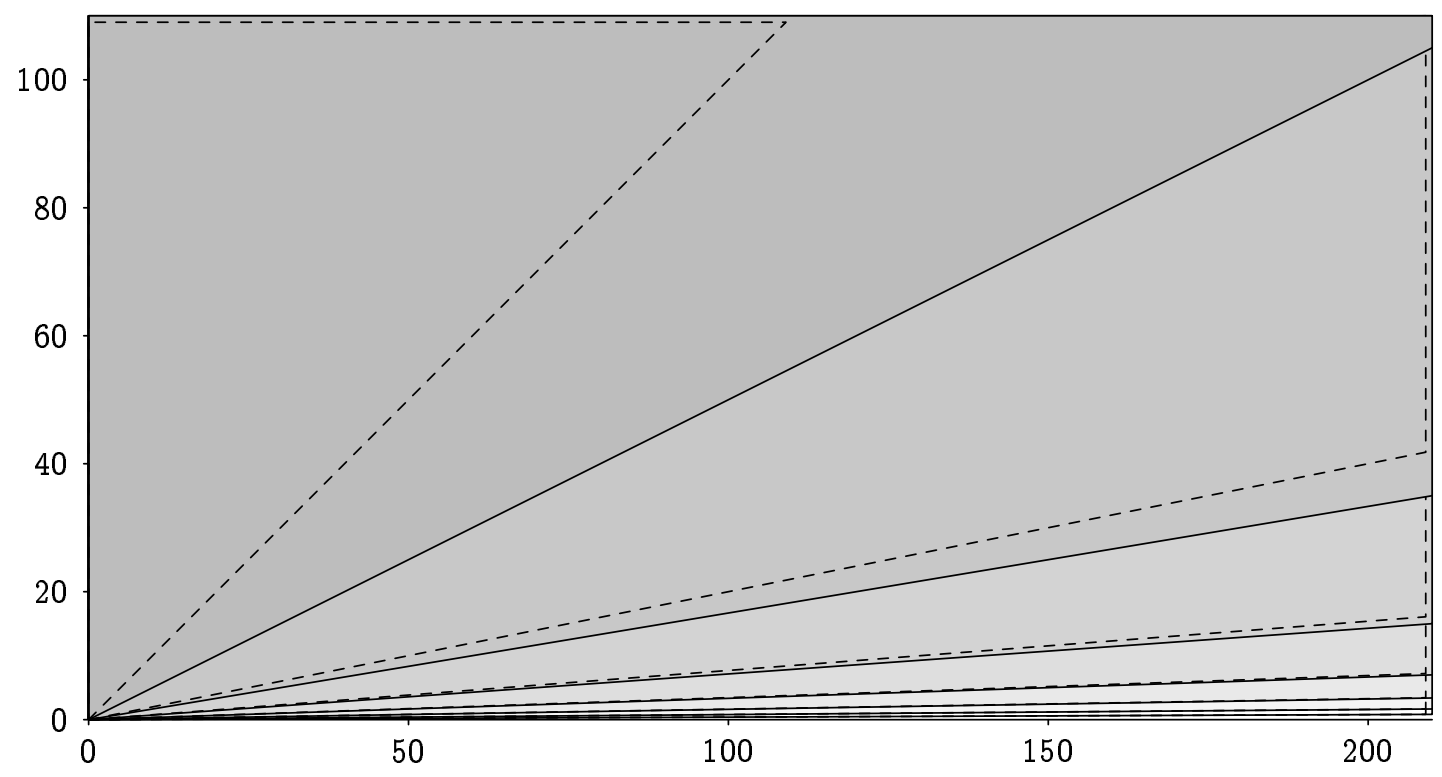

Abbildung 2.7: Bild zur Anwendbarkeit von Satz 2.2.19 für $p=2$. Gezeigt werden die Bereiche $t<\rho_{B} \cdot s$ für verschiedene $\rho_{B}$. Der Bereich ist umso dunkler, je größer $B$ genommen werden kann. Mit gestrichelten Linien sind die Teilbereiche hervorgehoben, in der die de-Luxe-Version 2.2.22 anwendbar ist.

$B^{u}$ unterscheidet sich von $B$ dadurch, daß die ersten $u$ Kästchen aus der $i_{0}$ ten Zeile entfernt worden sind. Die Folge $B^{0} \supset \cdots \supset B^{\pi\left(i_{0}\right)}$ sieht damit im Beispiel so aus:
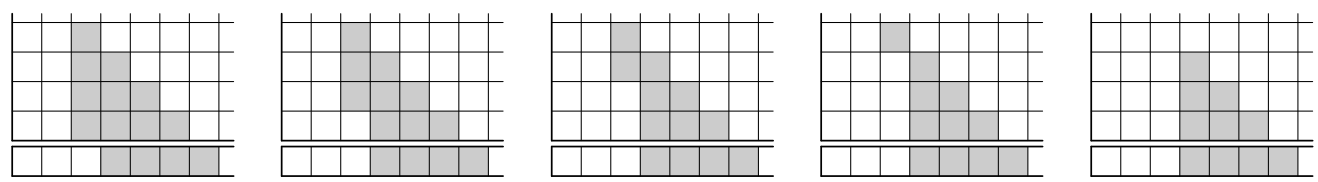

Die $B^{u}$ sind zwar für $1 \leq u<\pi\left(i_{0}\right)$ keine Hopf-Algebren, können aber trotzdem zur Zerlegung von $C_{*}$ benutzt werden. Dazu setzen wir $Q_{B^{u+1}, B^{u}}=P\left(0, \ldots, 0, p^{u}\right)$, $l_{B^{u+1}, B^{u}}=p$ und $T_{B^{u}}=Q(\tilde{\varepsilon}) P(\tilde{R})$ mit

$$
\tilde{\varepsilon}_{j}=\sigma(j)-1, \quad \tilde{r}_{i}= \begin{cases}0 & \text { für } i<i_{0}, \\ p^{\pi\left(i_{0}\right)}-p^{u}, & \text { für } i=i_{0}, \\ p^{\pi(i)}-1 & \text { für } i>i_{0} .\end{cases}
$$

Ist $0=B_{0} \subset \cdots \subset B_{N}=B^{\prime}$ eine Schrittzerlegung von $B^{\prime}$, so können wir die Sequenz $B^{\prime}=B^{\pi\left(i_{0}\right)} \subset \cdots \subset B^{u}$ anschließen; die Filtrierung

$$
0=B_{0} \subset \cdots \subset B_{N}=B^{\pi\left(i_{0}\right)} \subset \cdots \subset B^{u}
$$

nennen wir dann Schrittzerlegung von $B^{u}$. Wie oben sei

$$
\begin{aligned}
\rho_{B^{u}} & =\min \left\{\left|Q_{B_{m}, B_{m+1}}\right|: m=0, \ldots, N+\pi\left(i_{0}\right)-u-1\right\} \\
& =\min \left(\left\{\left|Q_{B_{m}, B_{m+1}}\right|: m=0, \ldots, N-1\right\} \cup\left\{\left|Q_{B^{v+1}, B^{v}}\right|: u \leq v<\pi\left(i_{0}\right)\right\}\right) .
\end{aligned}
$$

Damit gilt: 
Satz 2.2.22 (De-Luxe-Variante von 2.2.19). Lemma 2.2.11, die Korollare 2.2.12 und 2.2.13 und Satz 2.2.19 gelten weiter.

Beweis. Es reicht, das Schlüsselresultat Lemma 2.2.11 zu übertragen. Tatsächlich bleibt der gegebene Beweis einfach gültig. Die Details überlassen wir vertrauensvoll dem Leser.

Abbildung 2.7 zeigt für $p=2$ und für verschiedene $B$ die Bereiche, in denen Satz 2.2.19 oder Satz 2.2.22 anwendbar ist. Ein Beispiel für die Anwendung von Satz 2.2.19 sieht man in Abbildung 2.8.

\subsection{Der Algorithmus}

Aus den Sätzen 2.2.15 und 2.2.19 (bzw. der Verschärfung 2.2.22) kennen wir nun Kriterien, unter denen $2.18, \ldots, 2.20$ freundliche Sequenzen sind. Diese können also wie in Abschnitt 2.1.1 beschrieben zur Vereinfachung von Homologie- und Hebungsproblemen benutzt werden. Hier soll gezeigt werden, wie man daraus einen effektiven Algorithmus bastelt, der auch mit Erfolg auf zeitgenössischen Rechnern implementiert werden kann.

\subsubsection{Die Signaturfiltrierung}

Unser Ausgangspunkt ist eine zulässige Unteralgebra $B \subset A$ mit einer Schrittzerlegung $0=B\left(\Pi_{0}\right) \subset B\left(\Pi_{1}\right) \subset \cdots \subset B\left(\Pi_{N}\right)=B$. Sei

$$
\begin{aligned}
\mathfrak{S}_{B} & =\left\{\operatorname{sig}_{B}(Q(\varepsilon) P(R)) \mid Q(\varepsilon) P(R) \in B\right\} \\
& \cong\left\{(\varepsilon, R) \mid \varepsilon_{j}<\sigma(j), r_{i}<p^{\pi(i)} \forall j \geq 0, i \geq 1\right\}
\end{aligned}
$$

die Menge der möglichen $B$-Signaturen. Zur Abkürzung bezeichnen wir die $(\varepsilon, R) \in$ $\mathfrak{S}_{B}$ in diesem Abschnitt im Regelfall mit $\varpi$. Wie im Abschnitt vor Lemma 2.2.9 auf Seite 39 beschrieben, induziert eine Schrittzerlegung von $B$ eine Ordnung auf $\mathfrak{S}_{B}$ : es sei $\varpi<\varpi^{\prime}$, falls für ein $m$ die ersten $m$-Ziffern von $\varpi$ und $\varpi^{\prime}$ übereinstimmen während

$$
(m+1) \text { te Ziffer von } \varpi<(m+1) \text { te Ziffer von } \varpi^{\prime}
$$

gilt. Damit definieren wir

$$
\begin{aligned}
A^{[\varpi]} & =\mathbb{F}_{p}\left\{Q(\varepsilon) P(R) \mid \operatorname{sig}_{B}(Q(\varepsilon) P(R))=\varpi\right\}, \\
A^{[\geq \varpi]} & =\mathbb{F}_{p}\left\{Q(\varepsilon) P(R) \mid \operatorname{sig}_{B}(Q(\varepsilon) P(R)) \geq \varpi\right\} .
\end{aligned}
$$

Sei $\mathfrak{S}_{B}=\left\{\varpi_{0}, \ldots, \varpi_{K}\right\}$ eine Aufzählung der verschiedenen $B$-Signaturen mit $\varpi_{j}<$ $\varpi_{j+1}$. Die $A^{\left[\geq \varpi_{j}\right]}$ bilden eine absteigende Filtrierung

$$
A=A^{\left[\geq \varpi_{0}\right]} \supset A^{\left[\geq \varpi_{1}\right]} \supset \cdots \supset A^{\left[\geq \varpi_{K}\right]} \supset 0 .
$$

Wir nennen diese die Signaturfiltrierung; sie wird in unserer Implementierung des Algorithmus die Betrachtung der Sequenzen 2.18,..,2.20 ersetzen.

Zur Vereinfachung nehmen wir ab jetzt folgendes an: 
Annahme 2.3.1. Die Schrittzerlegung erfülle folgende Bedingungen:

- Jede $\sigma$-Ziffer sei signifikanter als jede $\pi$-Ziffer.

- Ein $\pi$-Kästchen sei signifikanter als alle $\pi$-Ziffern in höheren Zeilen.

Von den beiden in Abbildung 2.4 gezeigten Schrittzerlegungen erfüllt zum Beispiel die linke diese Annahme, die rechte aber nicht. Es gibt auch zu jedem $B$ eine Schrittzerlegung, die mit dieser Voraussetzung verträglich ist: eine solche erhält man beispielsweise, wenn man der Konstruktion aus dem Beweis von Lemma 2.2.8 folgt.

Lemma 2.3.2. Die $A^{[\geq \varpi]}$ sind Rechtsideale von $A$, dh. es ist $A^{[\geq \varpi]}=A^{[\geq \varpi]} \cdot A$.

Beweis. Wir müssen eine Multiplikation der Form $Q(\varepsilon) P(R) Q\left(\varepsilon^{\prime}\right) P\left(R^{\prime}\right)$ betrachten, bei der $\operatorname{sig}_{B}(Q(\varepsilon) P(R)) \geq \varpi$ ist. Man benutzt zunächst Lemma 1.1.14 und ersetzt ein auftretendes $P(R) Q_{s}$ durch $Q_{s} P(R)+\sum Q_{s+t} P\left(\ldots, r_{t}-p^{s}, \ldots\right)$. Hier kann die $B$-Signatur des Ausdrucks $Q_{s+t} P\left(\ldots, r_{t}-p^{s}, \ldots\right)$ nur dann kleiner als $P(R)$ sein, wenn das $p^{s}$, das an der tten Stelle abgezogen wird, zu einem $\pi$-Kästchen aus $B$ gehört. Also wäre $\pi(t)>s$, sodaß nach $2.12 \sigma(t+s)=2$ gilt. Das auftretende $Q_{s+t}$ erhöht damit eine $\sigma$-Ziffer, sodaß die $B$-Signatur insgesamt zunimmt.

Man muß also nur noch den Ausdruck $Q(\varepsilon) Q\left(\varepsilon^{\prime}\right) P(R) P\left(R^{\prime}\right)$ betrachten. Es ist zu zeigen, daß jeder Summand von $P(R) P\left(R^{\prime}\right)$ mindestens die $B$-Signatur des ersten Faktors hat. Dazu betrachte man eine Multiplikationsmatrix $X=\left(x_{i, j}\right)$ aus dem Milnor-Algorithmus. Falls

$$
x_{i, j} \equiv 0 \bmod p^{\pi(i)-j} \quad \text { für alle } i, j \geq 1
$$

gilt, so bleibt die $B$-Signatur unverändert: aus $r_{i}=\sum_{j} x_{i, j} p^{j}$ erhält man zunächst $r_{i} \equiv \sum_{j} x_{i, j} p^{j} \bmod p^{\pi(i)} \equiv x_{i, 0} \bmod p^{\pi(i)}$, sodaß man für den Summanden $P\left(t_{1}, \ldots, t_{n}\right)$ findet, daß $t_{n}=\sum_{i+j=n} x_{i, j} \equiv x_{n, 0} \bmod p^{\pi(n)} \equiv r_{n} \bmod p^{\pi(n)}$. Eine geänderte $B$ Signatur ergibt sich also nur, wenn 2.22 für mindestens ein Paar $i, j \geq 1$ verletzt ist.

Wir nehmen dies also probeweise an. Man wähle dann $i$ und $j$ so, daß $x_{i, j}$ minimale $p$-adische Bewertung hat: es sei $x_{i, j} \not \equiv 0 \bmod p^{k}$ mit einem $k \leq \pi(i)-j$, aber $x_{i^{\prime}, j^{\prime}} \equiv 0 \bmod p^{k-1}$ für alle $i^{\prime}, j^{\prime}$. Daraus ergeben sich wie oben $r_{n} \equiv x_{n, 0} \bmod p^{k}$ und $t_{n} \equiv r_{n} \bmod p^{k-1}$ für alle $n$. Daraus folgt zunächst, daß sich die Signaturen von $P(T)$ und $P(R)$ frühestens in der $k$ ten Zeile unterscheiden können. Und da $x_{i, j} \not \equiv 0 \bmod p^{k}$, hat $P(T)$ dort auch eine echt größere Ziffer stehen als $P(R)$.

Bemerkung 2.3.3. Aus dem Beweis sehen wir, daß der signaturerhaltende Teil der Multiplikation

$$
A^{[\varpi]} \subset A^{[\geq \varpi]} \stackrel{\cdot(\varepsilon) P(R)}{\longrightarrow} A^{[\geq \varpi]} \rightarrow A^{[\geq \varpi]} / A^{[>\varpi]} \cong A^{[\varpi]}
$$

direkt, dh. ohne den Umweg über die Bestimmung des vollen Produkts, berechnet werden kann: dazu lasse man bei der Benutzung der Kommutatorformel aus Lemma 1.1.14 einfach die Terme mit einem störenden $Q_{s+t}$ weg, und beschränke sich bei der Milnor-Multiplikation darauf, nur solche Multiplikationsmatrizen $X=\left(x_{i, j}\right)$ durchzugehen, die 2.22 erfüllen. 


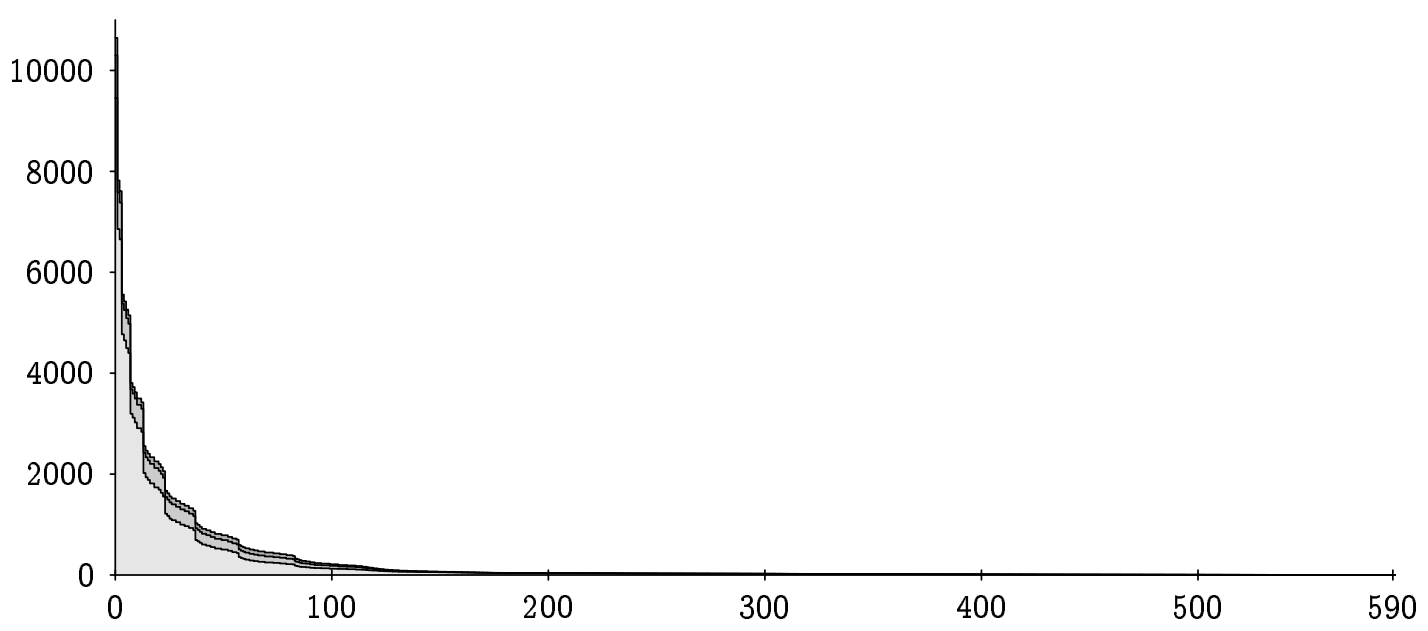

Abbildung 2.8: Zerlegung der Matrizen von $C_{35,245} \rightarrow C_{34,245} \rightarrow C_{33,245}$ für $p=2$ bei Verwendung von 2.2.22. Hier wurde $B(\pi, \sigma)$ mit $\pi=(0,0,4,3,2,1)$ und $\sigma=(0,0,0,1,1,1,1)$ benutzt. Alle Matrizen sind annähernd quadratisch. Vor der Zerlegung haben die Vektorräume Dimension $\approx 130000$. Bei der Zerlegung entstehen theoretisch $2^{14}=16384$ kleinere Matrizen, aber nur 590 von diesen haben von Null verschiedene Abmessungen. Die Mehrzahl der Matrizen ist sehr klein, deshalb bleiben ein paar ziemlich große Matrizen mit Größe bis zu 10700 übrig. Diese sind jedoch dem 130000-dimensionalen Ausgangsproblem immer noch bei weitem vorzuziehen.

Die Multiplikation $\cdot: B \otimes A \rightarrow A$ induziert einen Isomorphismus $B \otimes_{B} A \stackrel{\cong}{\longrightarrow} A$. Durch Einschränkung erhält man daraus

$$
B^{[\geq \varpi]} \otimes_{B} A \stackrel{\cdot}{\longrightarrow} A^{[\geq \varpi]} .
$$

Lemma 2.3.4. 2.23 ist ein Isomorphismus und es ist $A^{[\geq \varpi]} / A^{>\varpi} \cong \Sigma^{|\varpi|} B \backslash \backslash A$.

Beweis. Wir benutzen, daß $A$ nach Satz 2.2.1 ein freier $B$-Linksmodul ist. Deswegen ist $-\otimes_{B} A$ ein exakter Funktor, sodaß in dem Diagramm

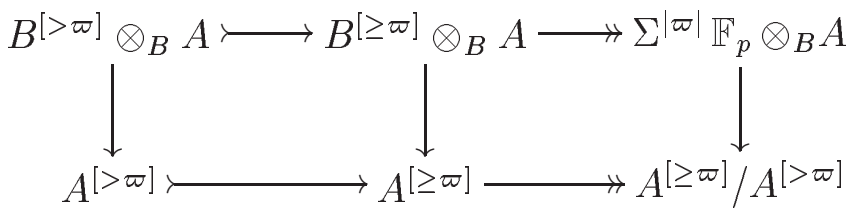

beide Zeilen exakt sind. 2.23 ist deswegen als Einschränkung von $B \otimes_{B} A \stackrel{\cong}{\longrightarrow} A$ immer injektiv. Es muß also nur die Dimensionsgleichheit beider Seiten gezeigt werden. Diese ergibt sich durch Induktion, da man leicht nachprüfen kann, daß $A^{[\geq \varpi]} / A^{>\varpi} \cong A^{[\varpi]}$ und $\Sigma^{|\varpi|} \mathbb{F}_{p} \otimes_{B} A$ in jedem Grad dieselbe Dimension haben. Daß $A^{[\geq \varpi]} / A^{>\varpi}$ und $\Sigma^{|\varpi|} B \backslash \backslash A$ identifiziert werden können ist damit auch klar (nämlich aus der rechten Spalte). 
Diese Aussagen über $A$ übertragen sich sofort auf eine jede $A$-freie partielle Auflösung $C_{*}$ : dazu setzen wir $C_{*}^{[\geq \varpi]}=A^{[\geq \varpi]} \cdot C_{*}$. Ist $G_{*} \subset C_{*}$ eine $A$-Basis so ist offenbar

$$
C_{*}^{[\geq \varpi]}=\mathbb{F}_{p}\left\{Q(\varepsilon) P(R) g \mid g \in G_{*}, \operatorname{sig}_{B}(Q(\varepsilon) P(R)) \geq \varpi\right\} .
$$

Passend dazu sei

$$
C_{*}^{[\varpi]}=\mathbb{F}_{p}\left\{Q(\varepsilon) P(R) g \mid g \in G_{*}, \operatorname{sig}_{B}(Q(\varepsilon) P(R))=\varpi\right\}
$$

obwohl dieses genaugenommen auch von der gewählten Basis $G_{*}$ abhängt. Wir erhalten somit die Signaturfiltrierung

$$
C_{*}=C_{*}^{\left[\geq \varpi_{0}\right]} \supset C_{*}^{\left[\geq \varpi_{1}\right]} \supset \cdots \supset C_{*}^{\left[\geq \varpi_{K}\right]} \supset 0 .
$$

Lemma 2.3.5. Das Differential $d: C_{*} \rightarrow C_{*-1}$ respektiert diese Filtrierung.

Beweis. Wegen $C_{*}^{[\geq \varpi]}=A^{[\geq \varpi]} \cdot C_{*}$, ist $d\left(C_{*}^{[\geq \varpi]}\right)=d\left(A^{[\geq \varpi]} \cdot C_{*}\right) \subset A^{[\geq \varpi]} \cdot d\left(C_{*}\right) \subset$ $C_{*-1}^{[\geq \varpi]}$.

Wie in Lemma 2.3.4 können wir auch in 2.24 die sukzessiven Quotienten $C_{*}^{[\geq \varpi]} / C_{*}^{[>\varpi]}$ mit dimensionsverschobenen Kopien von $\mathbb{F}_{p} \otimes_{B} C_{*} \cong T_{B} C_{*}$ identifizieren. Daraus ergibt sich der

Satz 2.3.6. Sei $C_{*}$ eine in $(S, T)$-erweiterbare Auflösung und $B \subset A$ wie oben eine zulässige Unteralgebra mit einer Schrittzerlegung. Sind die Voraussetzungen von Satz 2.2.15 oder 2.2.19/2.2.22 erfüllt, so sind

$$
C_{*}^{[\geq \varpi]} / C_{*}^{[>\varpi]} \longrightarrow C_{*} / C_{*}^{[>\varpi]} \longrightarrow C_{*} / C_{*}^{[\geq \varpi]}
$$

für $\varpi \neq 0$ freundliche exakte Sequenzen.

Beweis. Es ist zu zeigen, daß die Homologie der Quotienten $C_{*}^{[\geq \varpi]} / C_{*}^{[>\varpi]} \cong T_{B} C_{*}$ in unserem Bigrad verschwindet. Diese Quotienten können nach Lemma 2.3.4 mit denen, die bei Benutzung von 2.18,..,2.20 auftreten, identifiziert werden. Daß deren Homologie verschwindet, ist aber gerade die Aussage von Satz 2.2.15 bzw. $2.2 .19 / 2.2 .22$.

\subsubsection{Die Implementierung}

Aus Satz 2.3.6 erhält man nun ein Verfahren, das sich tatsächlich programmieren läßt. Dabei geht es uns um zwei eng verwandte Problemstellungen, die wir nacheinander diskutieren: die Berechnung der Auflösung und die Behandlung von Hebungsproblemen.

Sei zunächst $C_{*}$ eine in $(S, T)$ erweiterbare Auflösung. Man wähle ein $B \subset A$, auf das Satz 2.2.15 oder Satz 2.2.19/2.2.22 anwendbar sind, und eine Schrittzerlegung, die der Annahme des vergangenen Abschnitts genügt. Zur Fortsetzung der partiellen Auflösung im Bigrad $(S, T)$ gehe man so vor: 


\section{Algorithmus 2.3.7 (zur Erweiterung der Auflösung).}

$I$ Berechne die Homologie $H_{S, T}\left(C_{*} / C_{*}^{[>0]}\right)$.

Hier geht es gerade um den Signatur-Null-Anteil

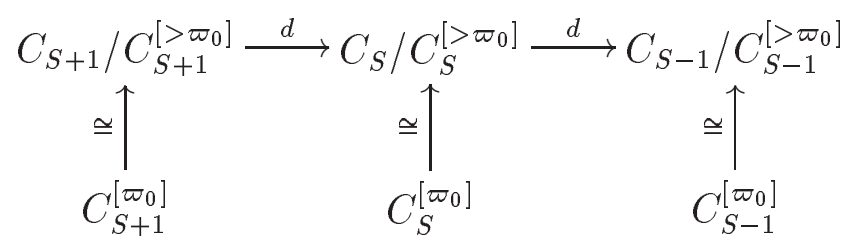

Bei der Berechnung beachte man Bemerkung 2.3.3. Das Resultat dieser Rechnung sind Vektoren $n_{1}, \ldots, n_{r} \in C_{S}$, die modulo $C_{S}^{[>0]}$ Zykel sind und eine Basis von $H_{S, T}\left(C_{*} / C_{*}^{[>0]}\right)$ repräsentieren.

Gehe nun die Menge $\mathfrak{S}_{B}=\left\{\varpi_{0}<\varpi_{1}<\cdots<\varpi_{K}\right\}$ der $B$-Signaturen der Reihe nach durch, beginnend mit $\varpi_{1}$.

$I I_{\varpi}$ Für ein solches $\varpi \in \mathfrak{S}_{B}$ schreibe man $d\left(n_{i}\right)=p_{i}+q_{i}$ wobei $p_{i} \in C_{S-1}^{[\varpi]}$ und $q_{i} \in C_{S-1}^{[>\varpi]}$.

Man berechne den signaturerhaltenden Teil

$$
C_{S+1}^{[\varpi]} \subset C_{S+1}^{[\geq \varpi]} \stackrel{d}{\longrightarrow} C_{S}^{[\geq \varpi]} \rightarrow C_{S}^{[\geq \varpi]} / C_{S}^{[>\varpi]} \cong C_{S}^{[\varpi]}
$$

des Differentials und hebe die $p_{i}$ durch diese Matrix: das Ergebnis sind Korrekturterme $c_{i} \in C_{S+1}^{[\varpi]}$ mit

$$
d\left(c_{i}\right)=p_{i}+\text { Terme größerer Signatur. }
$$

Man setze sodann $n_{i}^{\text {neu }}=n_{i}-c_{i}$ und gehe zum nächsten $\varpi$ über.

$I I I$ Man führe schließlich neue Erzeugende $g_{i}$ mit $d g_{i}=n_{i}$ in die Auflösung ein.

Begründung. Aufgrund der Freundlichkeit der 2.25 sind

$$
H_{S, T}\left(C_{*}\right)=H_{S, T}\left(C_{*} / C_{*}^{\left[>\varpi_{K}\right]}\right) \rightarrow H_{S, T}\left(C_{*} / C_{*}^{\left[>\varpi_{K-1}\right]}\right) \rightarrow \cdots \rightarrow H_{S, T}\left(C_{*} / C_{*}^{\left[>\varpi_{0}\right]}\right)
$$

sämtlich Isomorphismen. Die $n_{i}$ aus Schritt $I$ repräsentieren gerade eine Basis der letzten Gruppe; die Aufgabe der Korrekturschritte $I I_{\varpi_{1}}, \ldots, I I_{\varpi_{K}}$ ist es gerade, die $n_{i}$ zu Zykeln in $C_{*}$ zu deformieren, ohne die Homologieklassen zu ändern.

Beim Eintritt in Schritt $I I_{\varpi}$ kann man induktiv $d n_{i} \in C_{*}^{[\geq \varpi]}$ annehmen: Wegen

$$
0=d d n_{i}=d p_{i}+d q_{i} \equiv d p_{i} \bmod C_{*}^{[>\varpi]}
$$

ist $p_{i}$ in $C_{*}^{[\geq \varpi]} / C_{*}^{[>\varpi]}$ ein Zykel; aufgrund der Freundlichkeit von 2.25 ist $p_{i}$ dort also auch ein Rand, weswegen $c_{i}$ existiert. Damit ist $d n_{i}^{\text {neu }} \equiv p_{i}-p_{i}=0 \bmod C_{*}^{[>\varpi]}$, sodaß $n_{i}^{\text {neu }}$ die Induktionsannahme für den nächsten Durchgang erfüllt.

Sind schließlich $I I_{\varpi_{1}}, \ldots, I I_{\varpi_{K}}$ durchlaufen worden, so gilt $d n_{i} \in C_{*}^{\left[>\varpi_{K}\right]}=0$. Die $n_{i}$ sind somit Zykel und wir können - dem Standardalgorithmus folgend - neue Erzeugende $g_{i}$ mit Rand $n_{i}$ einführen. 
Hebungsprobleme lassen sich ganz ähnlich angehen: Angenommen $C_{*}$ sei bis $(S, T)$ exakt und es sei ein Zykel $z \in C_{S, T}$ gegeben, für den ein Lift $l$ aus $C_{S+1}$ gesucht wird.

Algorithmus 2.3.8 (zur Lösung eines Hebungsproblems). Man setze anfangs $l:=0, r:=z$. ( $r$ stehe für "Rest".) Gehe nun die Menge $\mathfrak{S}_{B}=\left\{\varpi_{0}<\varpi_{1}<\cdots<\right.$ $\left.\varpi_{K}\right\}$ der $B$-Signaturen der Reihe nach durch, beginnend mit $\varpi_{0}$.

$I_{\varpi}$ Man schreibe $r=p+q$ mit $p \in C_{S}^{[\varpi]}$ und $q \in C_{S}^{[>\varpi]}$. Man berechne den signaturerhaltenden Teil $C_{S+1}^{[\varpi]} \rightarrow C_{S}^{[\geq \varpi]} \rightarrow C_{S}^{[\varpi]}$ des Differentials und hebe $p$ durch diese Matrix: als Resultat erhält man ein $c \in C_{S+1}$ mit

$$
d(c)=p+\text { Terme größerer Signatur. }
$$

Setze nun $l^{\text {neu }}=l+c$ und $r^{\text {neu }}=r-d(c)$ und gehe zur nächsten Signatur über.

Begründung. Man überlegt sich leicht, daß $E:=d l+r$ bei den Durchgängen durch $I_{\varpi}$ erhalten bleibt. Da zu anfang offenbar $E=z$ gilt, muß man sich nur überlegen, daß am Ende der Rest $r$ verschwindet.

Tatsächlich gilt induktiv beim Eintritt in $I_{\varpi}$ jeweils $r \in C_{*}^{[\geq \varpi]}$. Da außerdem $d r=0$ gilt, ist $r$ in $C_{*}^{[\geq \varpi]} / C_{*}^{[>\varpi]}$ ein Zykel. Aufgrund der Freundlichkeit von 2.25 ist $r$ also modulo $C_{*}^{[>\varpi]}$ auch ein Rand, sodaß der gesuchte Korrekturterm $c$ existiert.

Hat $r$ schließlich sämtliche Schritte $I_{\varpi_{0}}, \ldots, I_{\varpi_{K}}$ durchlaufen, so gilt $r \in C_{*}^{\left[\varpi_{K}\right]}=$ 0 , wie gewünscht.

\subsubsection{Zur Wahl des passenden $B$}

Bleibt nur noch zu klären, wie man bei gegebenem $S$ und $T$ die am besten zum Bigrad $(S, T)$ passende Unteralgebra findet. Wir betrachten dazu die obere und die untere Variante zunächst getrennt. Sei also ein Bigrad $(S, T)$ gegeben.

Obere Variante (Satz 2.2.22). Hier gibt es ein eindeutig bestimmtes maximales $B$, das die Voraussetzung von Satz 2.2.22 erfüllt und keine unnützen Kästchen enthält. Hierbei sollen die Kästchen als unnütz gelten, deren Stellenwert größer ist als die betrachtete Dimension $T-S$. Da alle auftretenden Steenrod-Operationen in solchen Kästchen als Ziffer die Null stehen haben, kann unser Algorithmus dort nämlich keine Vereinfachung erbringen.

Das gesuchte $B=B(\pi, \sigma)$ ist explizit durch

$$
\begin{aligned}
& \sigma(j)= \begin{cases}2 & \text { falls } 2 p^{j}-1 \leq T-S \text { und } T<\left(2 p^{j}-1\right) \cdot S, \\
1 & \text { sonst },\end{cases} \\
& \pi(j)= \begin{cases}\min \left\{k \mid p^{k}\left(2 p^{j}-2\right) \geq T-S\right\} & \text { falls } T<\left(2 p^{j}-2\right) \cdot S, \\
0 & \text { sonst. }\end{cases}
\end{aligned}
$$

gegeben. Die einfache Verifizierung, daß dieses $B$ zulässig ist, überlassen wir dem Leser. Die Beziehungen $T<\left(2 p^{j}-1\right) \cdot S$ bzw. $T<\left(2 p^{j}-2\right) \cdot S$ stellen hierbei sicher, daß $T<\rho_{B} \cdot S$ gilt, sodaß Satz 2.2.22 anwendbar ist. Die Bedingungen, die sich auf 

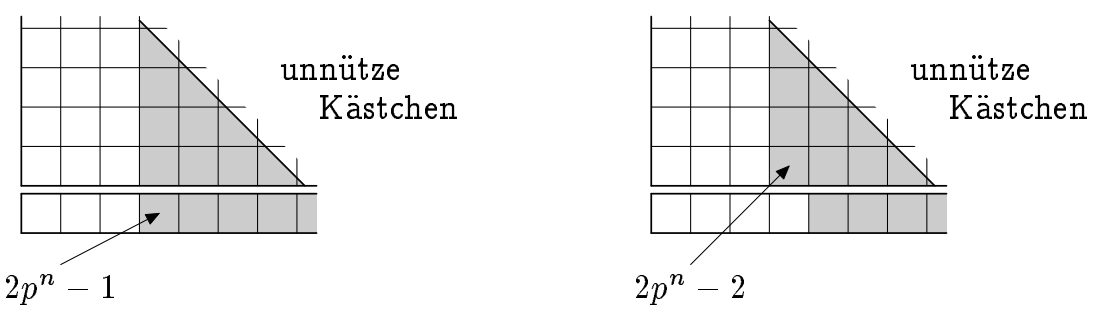

Abbildung 2.9: Bild zur Wahl von $B$ bei Anwendung von Satz 2.2.19 oder 2.2.22. Gezeigt wird ein typisches maximales $B$. Das Minimum in der Definition von $\rho_{B}$ wird jeweils in dem markierten Kästchen angenommen. Die unnützen Kästchen werden durch die Bedingung $2 p^{y}\left(p^{x}-1\right)>T-S$ charakterisiert, was näherungsweise der Halbebenen $y+x>\log _{p} \frac{1}{2}(T-S)$ entspricht.

$T-S$ beziehen, dienen der Vermeidung unnützer Kästchen. Man vergleiche dazu auch Abbildung 2.9. Dort erkennt man, daß $\rho_{B}$ hier immer von der Form $2 p^{j}-1$ oder $2 p^{j}-2$ ist. In Abbildung 2.7 wurden die zugehörigen Bereiche $T<\left(2 p^{j}-1\right) \cdot S$ und $T<\left(2 p^{j}-2\right) \cdot S$ für $p=2$ graphisch dargestellt.

Untere Variante (Satz 2.2.15). Hier sind in manchen Bigraden unterschiedliche maximale $B$ möglich. So sind zB. für $p=2$ und $(S, T)=(1,19)$ sowohl $B=B(1,1,1)$ mit $\tau_{B}=11, d_{B}=7$ wie auch $B=B(2,1)$ mit $\tau_{B}=6, d_{B}=3$ beide unter der Nebenbedingung $T-\tau_{B}>S \cdot d_{B}$ nicht erweiterbar.

Man sollte sich hier von der Philosophie leiten lassen, möglichst viele Kästchen mit kleinem Stellenwert zu benutzen: zum einen verteilen sich so die auftretenden Steenrod-Operationen gleichmäßiger; zum anderen vermeidet man so eine Vergrößerung der inversen Steigung $d_{B}$, was den erlaubten Bereich $T-\tau_{B}>S \cdot d_{B}$ schnell verkleinern würde. In der Praxis hat es sich bewährt die Kästchen nach folgendem Schema zu allozieren:

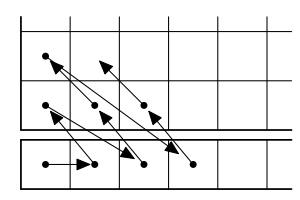

Für $p=2$ ergibt sich so zum Beispiel die Folge $B(1), B(1,1), B(2,1), B(2,1,1)$, $B(2,2,1), B(3,2,1), B(3,2,1,1)$, usw. Abbildung 2.6 zeigt für diese $B$ die Bereiche, in denen sie anwendbar sind.

Aus dem Vergleich der Abbildungen 2.6 und 2.7 sieht man, daß die obere Variante eher im oberen Teil des Quadranten, die untere dagegen eher im Unteren, anzuwenden ist. Welche bei gegebenem $(S, T)$ ratsam ist, entscheidet man am besten durch Ausprobieren: man bestimme dazu nach dem gerade geschilderten Verfahren das optimale obere bzw. untere $B$. $\mathrm{Zu}$ diesen errechne man jeweils die Dimensionen der Signatur-Null-Teile $C_{*}^{[0]}$. Sodann nehme man dasjenige $B$, welches hierfür den kleineren Wert liefert.

Wir wollen zum Schluß noch bemerken, daß somit zwar die Wahl von $B$ als einigermaßen kanonisch gelten kann, dasselbe aber nicht für die zusätzlich benötigte Schrittzerlegung gilt. Es scheint aber, daß deren Wahl das Laufverhalten des Algorithmus nicht erkennbar beeinflußt. 


\subsection{Resultate, Geschichte und Statistiken}

Das rechnerische Hauptresultat dieser Arbeit ist die Berechnung einer minimalen Auflösung der Steenrod-Algebra zur Primzahl 2. Mithilfe der grade geschilderten Theorie ist es möglich geworden, diese Auflösung im Bereich $t-s \leq 210$ explizit anzugeben. $\operatorname{Ext}_{A}^{s, t}\left(\mathbb{F}_{2}, \mathbb{F}_{2}\right)$ ist also jetzt für $t-s \leq 210$ bekannt (siehe Abbildung $2.10)$.

Das Problem der Berechnung von Ext hat eine lange Geschichte. Als Adams 1958 seine Spektralreihe definierte, war ihm sicherlich ein Anfangsstück schon bekannt. Der Durchbruch kam aber erst mit May's Dissertation von 1965 ([30], siehe auch [39], Ch. 3.2), in der eine Spektralreihe mit explizitem $E_{2}$-Term zur Berechnung von Ext angegeben wurde. Mithilfe dieser Spektralreihe gelang es dann Tangora ([44]) in 1970, den Ausgangsterm der Adams-Spektralreihe bis zur Dimension $70 \mathrm{zu}$ bestimmen.

Computerunterstützte Berechnungen sind schon sehr früh, nämlich in den 1960er Jahren, von Liulevicius versucht worden [25]. Nennenswerte Ergebnisse lieferten aber erst die Arbeiten von Bruner seit Mitte der 1980er Jahre. Ihm gelang es in [12] mittels des Standardverfahrens, $\operatorname{Ext}_{A}^{s, t}$ bis zu $t \leq 116$ zu berechnen. Später hat er diese Rechnungen bis zu $t \leq 140$ fortgesetzt [11]. Mit einem anderen Ansatz, nämlich der $\Lambda$-Algebra von Bousfield, Curtis, Kan, Quillen, Rector und Schlesinger (siehe [8]), hat Tangora in [45] die nicht-stabile Variante $\operatorname{UnExt}_{A}^{s}\left(\Sigma^{n+t} \mathbb{F}_{p}, \Sigma^{n} \mathbb{F}_{p}\right)$ für $t \leq 80$ untersucht.

Unsere Rechnungen haben wir auf einem 300MHz AMD-K6-2 Rechner unter GNU/Linux durchgeführt. Die nötigen Programme sind in C geschrieben worden und im Prinzip portabel. In den Abbildungen 2.12, . , 2.15 sind wichtige Kenngrößen der Rechnung graphisch dargestellt. Die berechnete Auflösung belegt ca. 1.7 Gigabyte in halb-komprimierter Form. In hohen Dimensionen brauchte das Programm mehr als 200MB RAM-Speicher, was aber glücklicherweise mittlerweile bezahlbar geworden ist. Die Berechnung dauerte insgesamt 108 Tage und erreichte Dimension 210. Die letzten 10 Dimensionen sind dabei Intel's Einführung der Multimedia Instruktionen im PC-Bereich [21] gedankt: mithilfe dieser MMX-Instruktionen lassen sich Milnorsche Basiselemente - zumindest für $p=2$ und bis zur Dimension 255 sehr effizient handhaben. Die Benutzung dieser Instruktionen hat die benötigte Rechenzeit annähernd halbiert. Die Ergebnisse unserer Rechnungen kann man zur Zeit unter [38] abrufen. Allerdings wird sich diese Adresse wohl in absehbarer Zukunft ändern.

Wir bemerken noch, daß man auch die Kohomologie von Unter-Hopfalgebren der Steenrod-Algebra mit den Programmen berechnen kann. Abbildung 2.11 zeigt zB. die Kohomologie der Unteralgebra $B(3,2,1)$, die auch unter der Bezeichnung $A(2)$ bekannt ist. $\operatorname{Ext}_{A(2)}^{*, *}\left(\mathbb{F}_{2}, \mathbb{F}_{2}\right)$ ist allerdings schon seit langem bekannt: es wurde 1967 von Shimada und Iwai in [41] vollständig berechnet. In jüngster Zeit ist Ext E(2) im Zusammenhang mit der Theorie der topologischen Modulformen [20] wichtig geworden. 


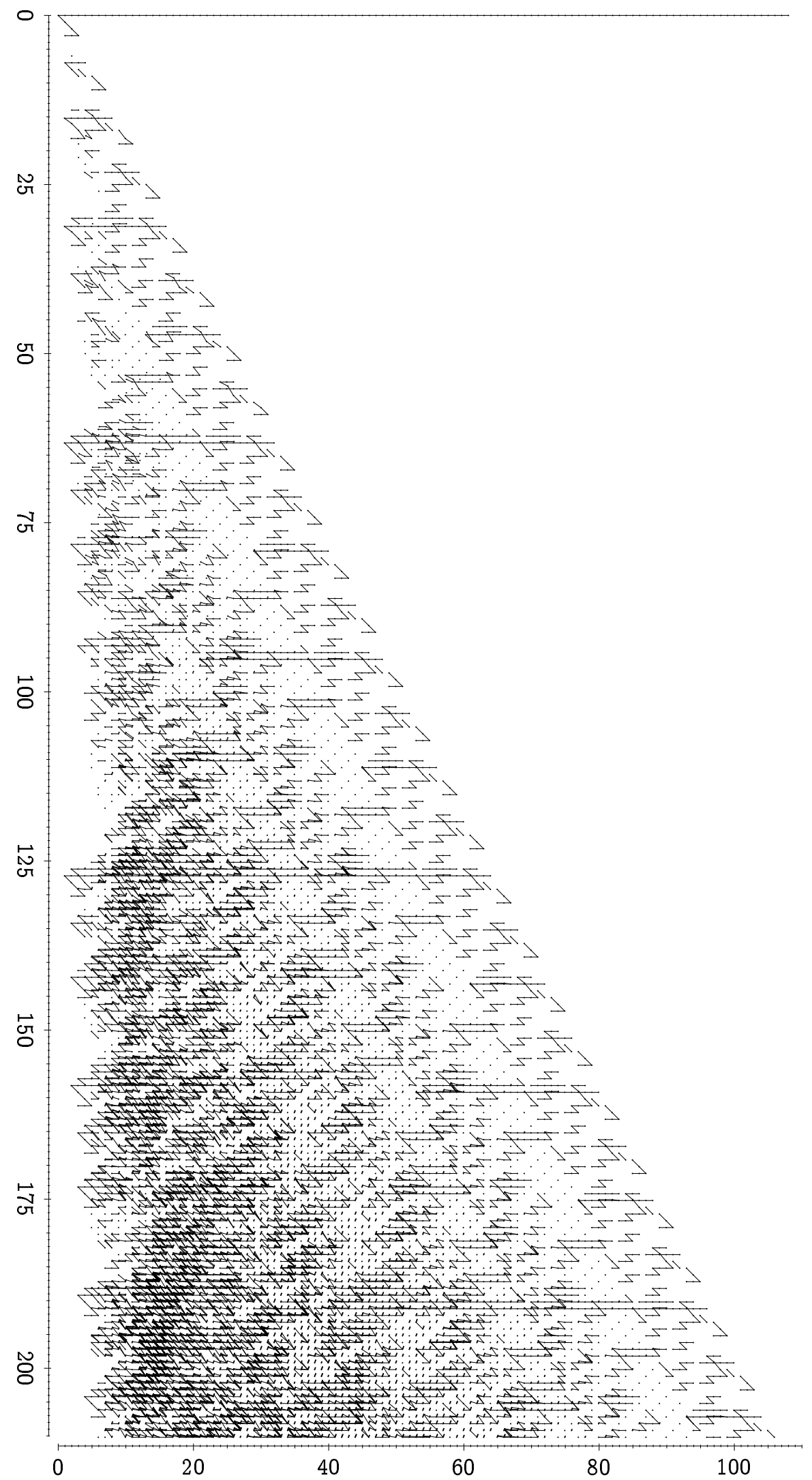

Abbildung 2.10: Panorama-Blick auf $\operatorname{Ext}_{A}^{* * *}\left(\mathbb{F}_{2}, \mathbb{F}_{2}\right)$ bis zur Dimension 210. 


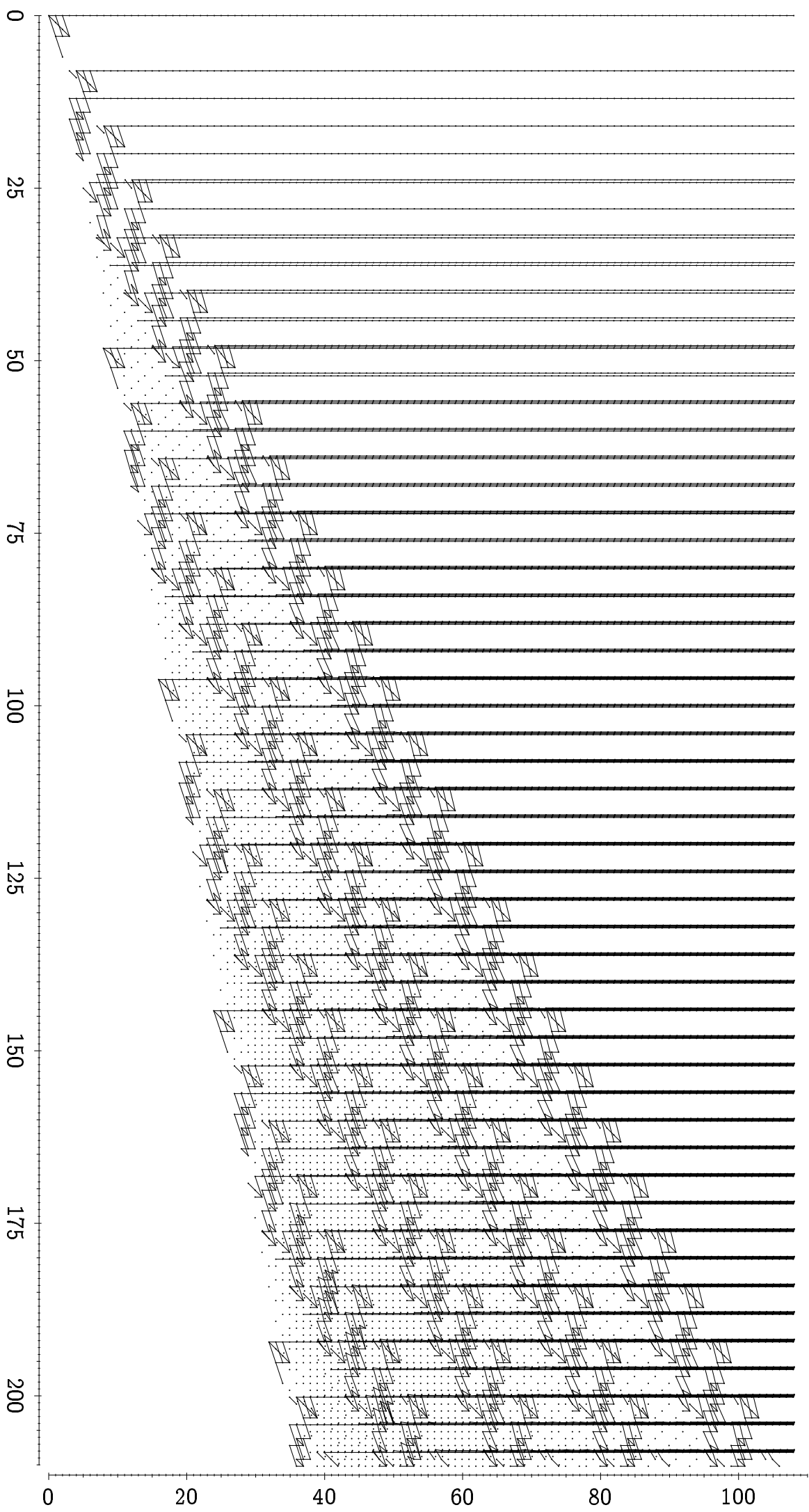

Abbildung 2.11: Panorama-Blick auf $\operatorname{Ext}_{A(2)}^{*, *}\left(\mathbb{F}_{2}, \mathbb{F}_{2}\right)$ bis zur Dimension 210. 


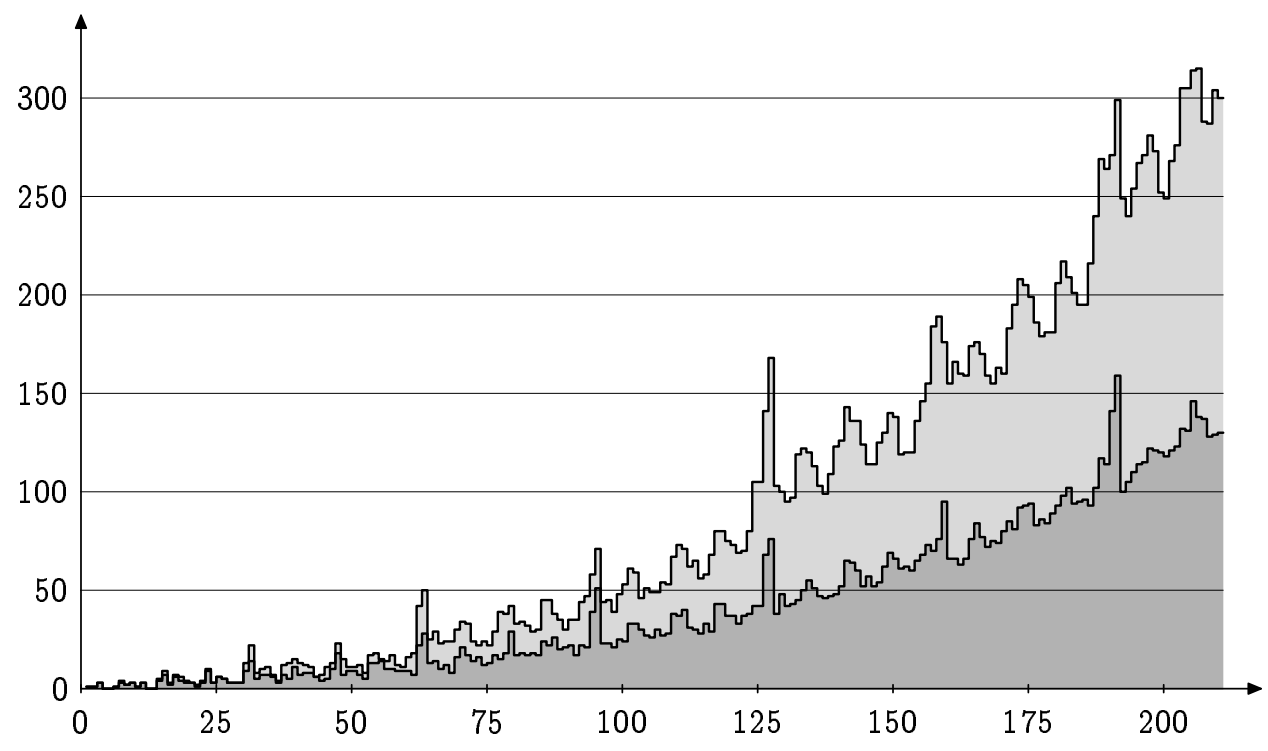

Abbildung 2.12: Dimension von $\operatorname{Ext}_{A}^{s, t}\left(\mathbb{F}_{2}, \mathbb{F}_{2}\right)$ für $n=t-s \leq 210$. Insgesamt gibt es 19581 Erzeugende. Der dunklere Bereich zeigt die Zahl der Erzeugenden im Bereich $5 s \geq n$.

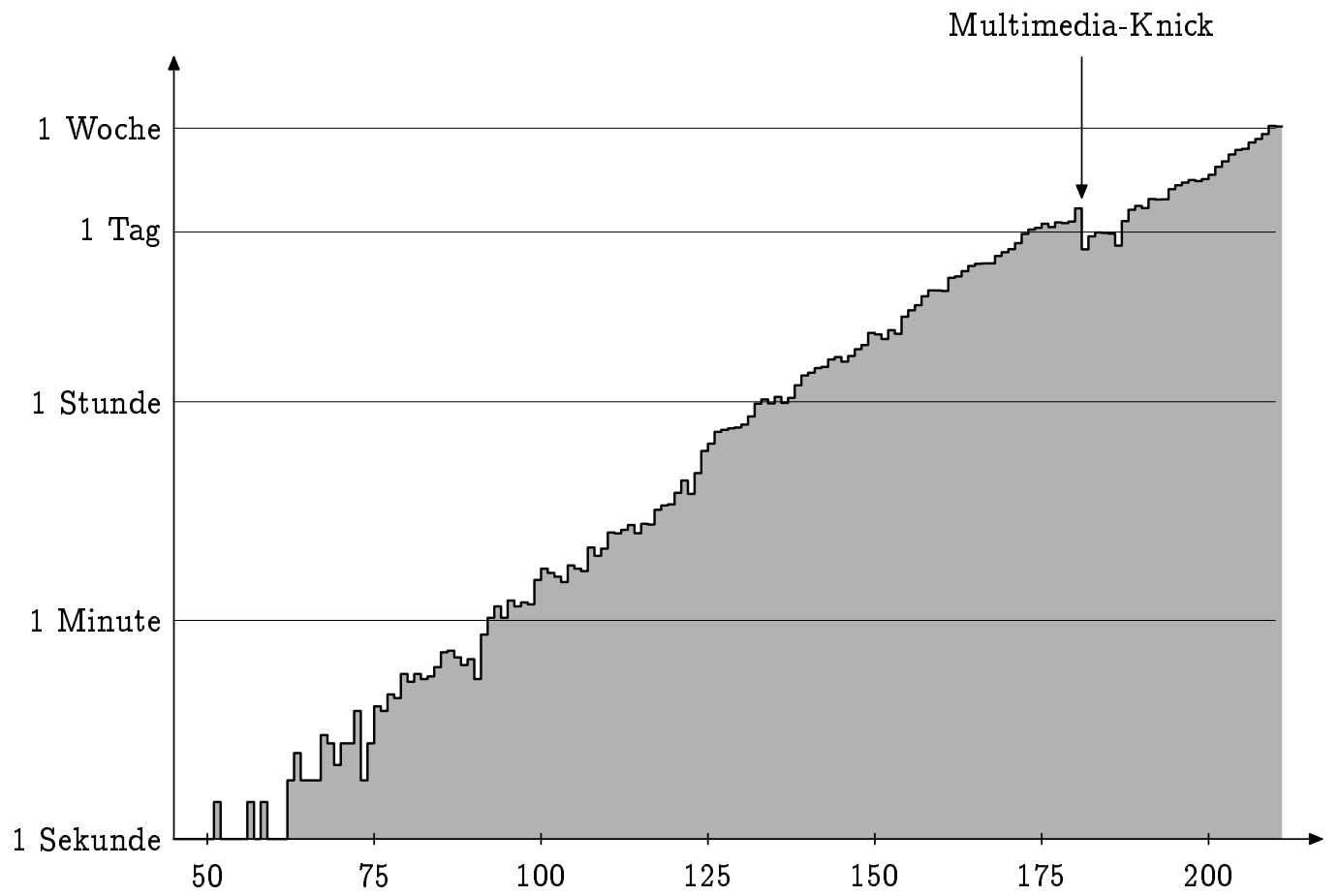

Abbildung 2.13: Rechenzeit für die Berechnung der minimalen Auflösung für $p=2$. Die Gesamtzeit betrug 108 Tage und 18 Stunden auf einem 300MHz AMD K6-2. Die Zeit pro Dimension verdoppelt sich alle 10 Dimensionen. In Dimension 181 kann man sehr schön den "Multimedia-Knick" erkennen: dort wurde die Multiplikationsroutine in Assembler umgeschrieben, unter Benutzung der $\mathrm{MMX}^{(\mathrm{TM})}$-Instruktionen; die Rechenzeit halbierte sich daraufhin. 


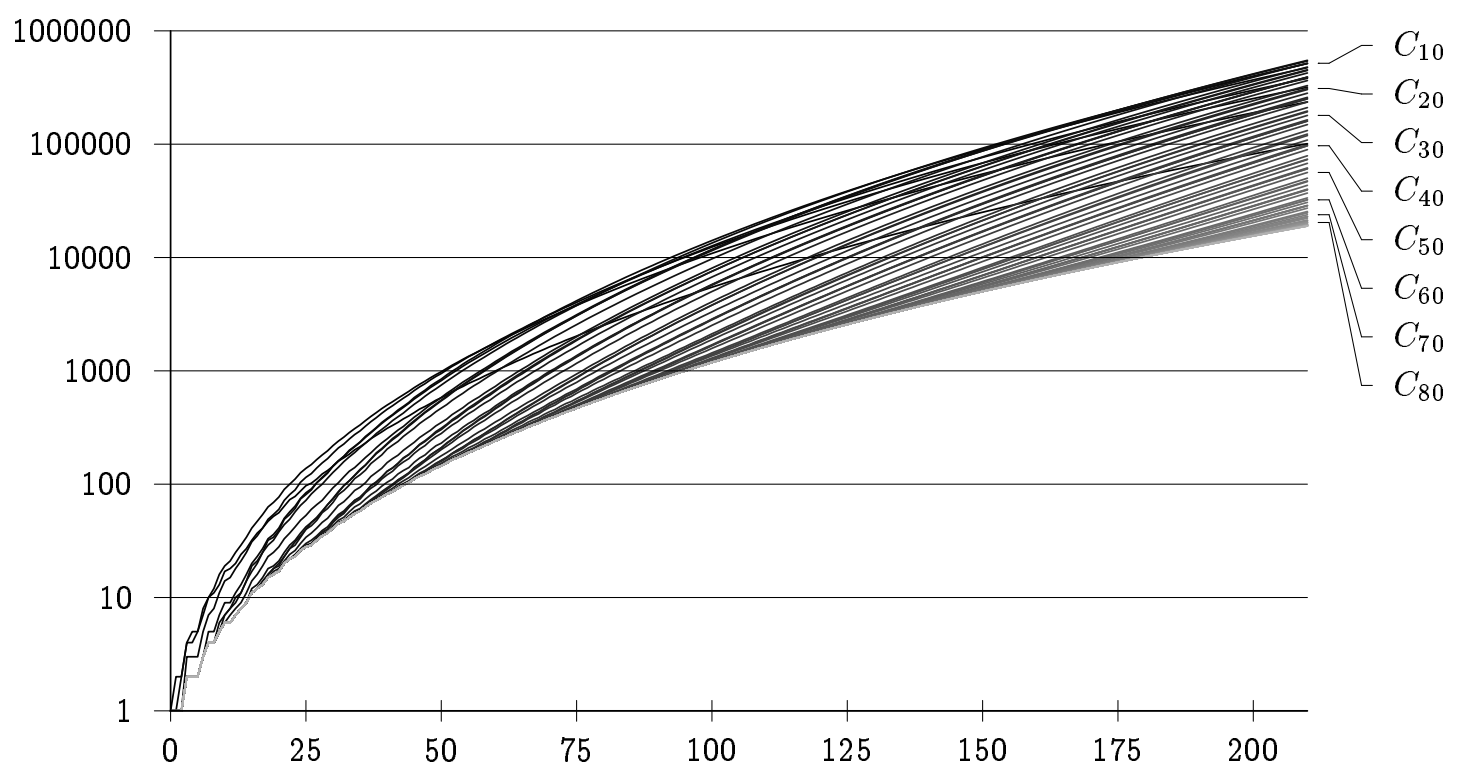

Abbildung 2.14: Wachstum der minimalen Auflösung für $p=2$. Gezeigt wird $\operatorname{dim}_{\mathbb{F}_{2}} C_{s, s+n}$ in Abhängigkeit von $n \leq 210$. $C_{1}$ entspricht der etwas dunkleren Linie in der Mitte, die ein noch moderates Wachstum zeigt. Die Dimensionen werden dann bis circa $C_{11}$ größer, bevor sie für $s \rightarrow \infty$ auf die Größe der Steenrod-Algebra selber zurückfallen. $C_{11,221}$ hat übrigens Dimension $\geq 500000$.

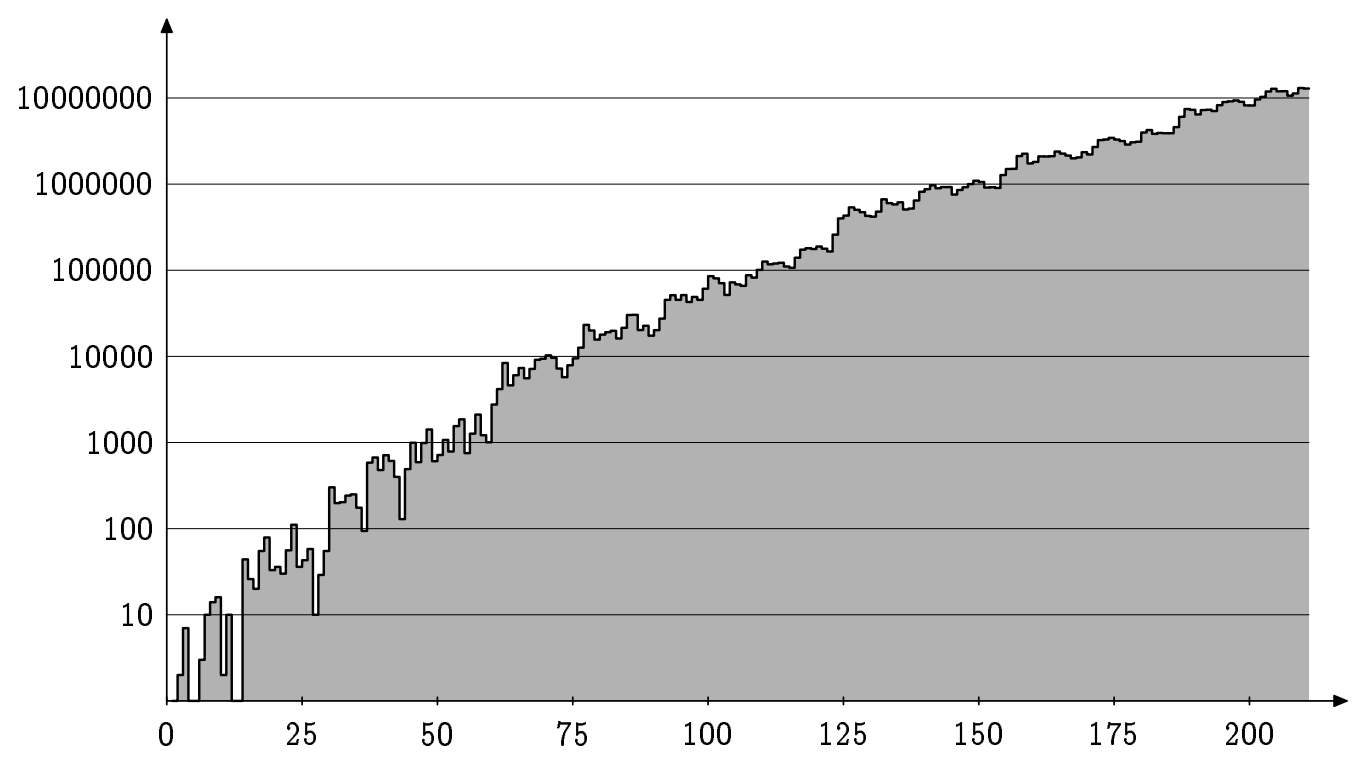

Abbildung 2.15: Größe der Beschreibung der minimalen Auflösung für $p=2$. Gezeigt wird zu jeder Dimension $n \leq 210$ die Gesamtzahl der Milnor-Basiselemente in den Differentialen $d(g)$ für die $A$-Erzeugenden $g \in C_{s, s+n}$. Setzte man also Abbildung 1.7 in höhere Dimensionen fort, so wäre dies die Anzahl der Summanden, die in der rechten Spalte stehen müßten. Insgesamt stünden dort 342223927 Summanden. 
2 Ein Lemma 


\section{Weitere Anwendungen}

Die über 100 Tage Rechenzeit, die in die Berechnung der minimalen Auflösung investiert worden sind, und die immense Datenmenge, die dabei angefallen ist, erscheinen im Vergleich zur bisherigen Ausbeute noch unverhältnismäßig. Wir zeigen aber in diesem Kapitel, daß man aus diesen Daten mit relativ geringem Aufwand viele weitere interessante Resultate gewinnen kann.

\section{1 $\operatorname{Ext}_{A}(M, N)$ für kleine $M$ und $N$}

Lemma 1.3.2 zufolge kann man $\operatorname{Ext}_{A}^{s, t}(M, N)$ für beliebige $A$-Moduln $M$ und $N$ aus einer Auflösung des trivialen Moduls $k$ berechnen. Mit gewissen Einschränkungen gilt dies auch für partielle Auflösungen. Für einen $A$-Modul $M$ seien $d_{M}, D_{M} \in$ $\mathbb{Z} \cup\{ \pm \infty\}$ durch

$$
\begin{aligned}
d_{M} & =\max \left\{d \mid M_{t}=0 \text { für } t<d\right\}, \\
D_{M} & =\min \left\{D \mid M_{t}=0 \text { für } t>D\right\}
\end{aligned}
$$

definiert.

Lemma 3.1.1. Sei $M$ ein $A$-Modul und $C_{*}$ eine partielle Auflösung von $k$ über $A$ bis $(S, T)$. Dann ist $C_{*} \wedge M$ eine partielle Auflösung von $M$ bis $\left(S, T+d_{M}\right)$.

Beweis. Wegen Lemma 1.1.5 ist $C_{*} \wedge M$ jedenfalls ein Komplex freier $A$-Moduln. Aus der Künneth-Formel

$$
H_{s, t}\left(C_{*} \wedge M\right)=\left(H_{s, *}\left(C_{*}\right) \wedge M\right)_{t}=\bigoplus_{t^{\prime}+t^{\prime \prime}=t} H_{s, t^{\prime}}\left(C_{*}\right) \otimes M_{t^{\prime \prime}}
$$

folgt zunächst $H_{0}\left(C_{*} \wedge M\right)=H_{0}\left(C_{*}\right) \wedge M=k \wedge M \cong M$. Da $H_{s, t}\left(C_{*}\right)=0$ für $0<s \leq S$ und $t \leq T$, folgt außerdem $H_{s, t}\left(C_{*} \wedge M\right)=0$ für $0<s \leq S$ und $t \leq T+d_{M}$, denn ein nichttriviales $M_{t^{\prime \prime}}$ hat immer $t^{\prime \prime} \geq d_{M}$. Also ist $C_{*}$ eine partielle Auflösung von $M$ bis $\left(S, T+d_{m}\right)$.

Korollar 3.1.2. Ist $C_{*}$ eine partielle Auflösung von $k$ über $A$ bis $(S, T)$, so ist für $s<S$

$$
\begin{array}{ll}
\operatorname{Ext}_{A}^{s, t}(M, N)=H_{s, t}\left(\operatorname{Hom}_{A}\left(C_{*} \wedge M, N\right)\right) & \text { für } t \leq T+d_{M}-D_{N}, \\
\operatorname{Tor}_{s, t}^{A}(M, N)=H_{s, t}\left(M \otimes_{A}\left(C_{*} \wedge N\right)\right) & \text { für } t \leq T+d_{M}+d_{N} .
\end{array}
$$

Falls $C_{*}$ sogar minimal ist, kann man hier $s<S$ durch $s \leq S$ ersetzen. 
Beweis. Man kann $C_{*}$ zu einer vollständigen Auflösung $\tilde{C}_{*}$ fortsetzen, und zwar so, daß man eine injektive Vergleichsabbildung $\phi_{*}: C_{*} \longmapsto \tilde{C}_{*}$ hat, die im Bereich $R_{S, T}$ ein Isomorphismus ist. Dann hat man kurze exakte Sequenzen

$$
\begin{aligned}
\operatorname{Hom}_{A}\left(\operatorname{coker} \phi_{*} \wedge M, N\right) & \longmapsto \operatorname{Hom}_{A}\left(\tilde{C}_{*} \wedge M, N\right) \rightarrow \operatorname{Hom}_{A}\left(C_{*} \wedge M, N\right), \\
M \otimes_{A}\left(C_{*} \wedge N\right) & \longmapsto M \otimes_{A}\left(\tilde{C}_{*} \wedge N\right) \rightarrow M \otimes_{A}\left(\operatorname{coker} \phi_{*} \wedge N\right) .
\end{aligned}
$$

Da coker $\phi_{*}$ auf $R_{S, T}$ null ist, verschwindet

$$
\begin{array}{ll}
\operatorname{Hom}_{A}^{s, t}\left(\operatorname{coker} \phi_{*} \wedge M, N\right) & \text { für }(s, t) \in R_{S, T+d_{M}-D_{N}}, \\
\left(M \otimes_{A}\left(\operatorname{coker} \phi_{*} \wedge N\right)\right)_{s, t} & \text { für }(s, t) \in R_{S, T+d_{M}+d_{N}} .
\end{array}
$$

Die Behauptung folgt damit aus den zugehörigen langen exakten Homologiesequenzen. Die Verschärfung im Fall eines minimalen $C_{*}$ überlassen wir dem Leser als Übungsaufgabe.

Falls $M$ und $N$ endliche $A$-Moduln von kleiner Dimension sind, so ist Lemma 3.1.1 auch ein sehr effizientes Verfahren zur Berechnung von $\operatorname{Ext}_{A}^{s, t}(M, N)$. Dies wollen wir im folgenden genauer ausführen. Sei $G_{s} \subset C_{s}$ für jedes $s$ eine $A$-Basis. In der Praxis ist $C_{*}$ gerade wie in Abbildung 1.7 durch eine solche Basis $G_{*}$ zusammen mit einer Tabelle der Differentiale $d g \in C_{s-1}$ für $g \in G_{s}$ gegeben. Seien außerdem $\left\{m_{1}, \ldots, m_{p}\right\} \subset M$ und $\left\{n_{1}, \ldots, n_{q}\right\} \subset N$ Vektorraumbasen. Nach Lemma 1.1.5 ist $C_{s} \wedge M$ über $A$ frei mit Basis $\left\{g \wedge m_{i} \mid g \in G_{s}, 1 \leq i \leq p\right\}$. Definiert man $\phi_{g, i}^{j}: C_{s} \wedge M \rightarrow N$ als $A$-lineare Fortsetzung von $\phi_{g, i}^{j}\left(g^{\prime} \wedge m_{i^{\prime}}\right)=\delta_{g, g^{\prime}} \delta_{i, i^{\prime}} n_{j}$, so ist also

$$
\operatorname{Hom}_{A}\left(C_{s} \wedge M, N\right)=k\left\{\phi_{g, i}^{j} \mid g \in G_{s}, 1 \leq i \leq q, 1 \leq j \leq p\right\} .
$$

Nun muß man nur noch das Differential $\left(d_{s} \wedge \mathrm{id}\right)^{*}: \operatorname{Hom}_{A}\left(C_{s-1} \wedge M, N\right) \rightarrow \operatorname{Hom}_{A}\left(C_{s} \wedge\right.$ $M, N)$ in dieser Basis als Matrix darstellen. Es ist also $\phi_{g^{\prime}, i^{\prime}}^{j^{\prime}}\left(d g \wedge m_{i}\right)$ für diverse $g, g^{\prime}, i, i^{\prime}, j^{\prime}$ zu berechnen. Schreibt man $d g^{\prime}=\sum_{g^{\prime \prime}} a_{g^{\prime}, g^{\prime \prime}} g^{\prime \prime}$ mit $a_{g^{\prime}, g^{\prime \prime}} \in A$, so muß man Ausdrücke der Form $\phi_{g^{\prime}, i^{\prime}}^{j^{\prime}}(a g \wedge m)$ auswerten. Dazu benutzt man die Beziehung

$$
(a g) \wedge m=\sum a^{\prime}\left(g \wedge \chi\left(a^{\prime \prime}\right) m\right)
$$

die im Beweis von Lemma 1.1.5 angegeben wurde. Diese leitet sich aus

$$
\begin{aligned}
\sum a^{\prime}\left(g \wedge \chi\left(a^{\prime \prime}\right) m\right) & =\sum\left(a^{\prime} g\right) \wedge a^{\prime \prime} \chi\left(a^{\prime \prime \prime}\right) m \\
& =\sum\left(a^{\prime} g\right) \wedge \varepsilon\left(a^{\prime \prime}\right) m \\
& =(a g) \wedge m
\end{aligned}
$$

her. Man erhält somit

$$
\phi_{g, i}^{j}\left((a g) \wedge m_{i^{\prime}}\right)=\sum \phi_{g, i}^{j}\left(a^{\prime}\left(g \wedge \chi\left(a^{\prime \prime}\right) m_{i}\right)\right)=\sum a^{\prime} \phi_{g, i}^{j}\left(g \wedge \chi\left(a^{\prime \prime}\right) m_{i}\right),
$$

woraus die Matrixdarstellung abgelesen werden kann. 
Bemerkung 3.1.3. Ein Vorzug dieser Methode liegt darin, daß die Zuordnung $M \mapsto C_{*} \wedge M$ ein Funktor ist. Ist $\phi: M \rightarrow M^{\prime}$ eine $A$-lineare Abbildung, so ist id $\wedge \phi: C_{*} \wedge M \rightarrow C_{*} \wedge M^{\prime}$ eine Kettenabbildung, die $\phi$ hebt. Also kann man bei gegebenem $\psi: N^{\prime} \rightarrow N$ die induzierte Abbildung

$$
\psi_{*} \phi^{*}: \operatorname{Ext}_{A}^{s, t}\left(M^{\prime}, N^{\prime}\right) \rightarrow \operatorname{Ext}_{A}^{s, t}(M, N)
$$

einfach aus

$$
\psi_{*} \phi^{*}: \operatorname{Hom}_{A}^{s, t}\left(C_{*} \wedge M^{\prime}, N^{\prime}\right) \rightarrow \operatorname{Hom}_{A}^{s, t}\left(C_{*} \wedge M, N\right)
$$

berechnen.

Bemerkung 3.1.4. Hat $M$ eine zusätzliche Struktur, zB. eine Filtrierung, so wird diese auf $C_{*} \wedge M$ vererbt. Beispielsweise hat jeder $A$-Modul die Skelettfiltrierung

$$
\cdots \subset M^{\geq k-1} \subset M^{\geq k} \subset M^{\geq k+1} \subset \cdots
$$

mit

$$
M^{\geq k}=\{m \in M:|m| \geq k\} .
$$

Die sukzessiven Quotienten $M^{\geq k} / M^{\geq k+1} \cong M_{k}$ haben die triviale $A$-Modulstruktur, sodaß sie zu einer direkten Summe von trivialen Moduln $\Sigma^{k} k$ isomorph sind. Man erhält somit eine Spektralreihe vom Atiyah-Hirzebruch-Typ

$$
E_{1}^{s, t}=\bigoplus_{t^{\prime}+t^{\prime \prime}=t} \operatorname{Tor}_{s, t^{\prime}}^{A}(k, k) \otimes M_{t^{\prime \prime}} \Rightarrow \operatorname{Tor}_{s, t}^{A}(M, k)
$$

die in der Literatur häufig zur Berechnung eingesetzt wird. Die grade geschilderte Methode ist mit dieser eng verwandt. Da $G_{s}$ mit einer $k$-Basis von $\operatorname{Tor}_{s, *}^{A}(k, k)$ identifiziert werden kann, ist ja

$$
\operatorname{Hom}_{A}\left(C_{s} \wedge M, k\right) \cong \operatorname{Hom}_{k}\left(\operatorname{Tor}_{s, *}^{A}(k, k) \otimes M, k\right) .
$$

Also beginnen beide Verfahren mit derselben oberen Schranke.

\subsubsection{Beispiele}

Zur Illustration der Effizienz dieses Verfahrens geben wir in den Abbildungen $3.1, \ldots$, 3.6 für $p=2$ ein paar Beispiele an. Jede dieser Kohomologie-Karten konnte innerhalb weniger Stunden erstellt werden. Würde man dagegen zur Berechnung von $\operatorname{Ext}_{A}^{s, t}(M, k)$ erst eine jeweils neue Auflösung von $M$ berechnen, so müßte man für jedes Bild wiederum ca. 100 Tage veranschlagen. Die Moduln, deren Kohomologie in den Abbildungen gezeigt wird, sind folgende:

Der Fragezeichenkomplex $F$ (englisch: questionmark-complex, Abbildung 3.2 auf Seite 67): Als Vektorraum ist

$$
F_{t}= \begin{cases}\mathbb{F}_{2} & t=0,1,3, \\ 0 & \text { sonst }\end{cases}
$$


Seien $b \in F_{0}, m \in F_{1}$ und $t \in F_{3}$ die von Null verschiedenen Elemente. Damit sei $\mathrm{Sq}^{1} b=m, \mathrm{Sq}^{2} m=t . F$ spielt in der $K$-lokalen Homotopietheorie eine besondere Rolle, siehe [7], 10.7. Der Name erklärt sich aus der gebräuchlichen bildlichen Darstellung

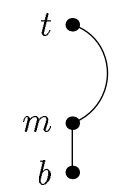

Mitchells $A(n)$-freie $A$-Moduln für $n=0,1,2$. Dazu sei $A(n) \subset A$ die Hopf-Unteralgebra $B(n+1, n, \ldots, 2,1)$. Mitchell hat in [36] gezeigt, daß man für jedes $n$ die $A(n)$-Operation auf $A(n)$ zu einer $A$-Operation fortsetzen kann. Mit anderen Worten findet man $A$-Moduln $A_{n}$, sodaß $A_{n}$ über $A(n)$ zu $A(n)$ isomorph ist. Es gibt genau eine Wahl für $A_{-1}$ und $A_{0}, 4$ Wahlen für $A_{1}$ und 1600 für $A_{2}$. Für jeweils einen solchen Modul wird in den Abbildungen 3.1, 3.3 und 3.4 die Kohomologie gezeigt.

Die $A_{n}$ sind für $n \leq 2$ als Kohomologie von Spektren realisierbar: man hat $H^{*}\left(S^{0}\right)=A_{-1}, H^{*}\left(\Sigma^{-1} \mathbb{R} P^{2}\right)=A_{0}$. Für die Realisierbarkeit von $A_{1}$ und $A_{2}$ kann man den folgenden Satz benutzen:

Satz 3.1.5. Sei $M$ ein endlicher $A$-Modul, der über $A(0)$ frei ist. Falls $\operatorname{Ext}_{A}^{3, t}\left(M, \mathbb{F}_{p}\right)$ für $t \leq D_{M}+1$ verschwindet, so gibt es ein Spektrum $X$ mit $H^{*}(X)=M$.

Beweis. Siehe [29], Korollar 23 b), Ch. 16, Seite 271.

Für die von uns betrachteten Versionen von $A_{1}$ und $A_{2}$ kann man also folgendermaßen schließen: Aus Abbildung 3.3 sieht man, daß $\operatorname{Ext}_{A}^{3, t}\left(A_{1}, \mathbb{F}_{2}\right)$ für $t \leq 14$ Null ist. Da $D_{A_{1}}=6$, ist $A_{1}$ also realisierbar (siehe auch [29], Ch 16., oder [16].) Genauso sieht man aus Abbildung 3.4, daß $\operatorname{Ext}_{A}^{3, t}\left(A_{2}, \mathbb{F}_{2}\right)=0$ für $t \leq 30$. Wegen $D_{A_{2}}=23$, ist also auch unser $A_{2}$ realisierbar. Wir vermuten, daß sich jedes $A_{2}$ auf diese Weise realisieren läßt, haben dies aber noch nicht nachgerechnet. Über die Realisierbarkeit $\operatorname{der} A_{n}$ für $n \geq 3$ ist nichts bekannt.

Wie Mitchell in [36] (Bemerkung auf Seite 238-239) festgestellt hat, kann man eine Version von $A_{1}$ aus der kompakten Lie-Gruppe $G_{2}$ konstruieren. Dazu sei $C_{2}^{3} \subset G_{2}$ ein maximaler 2-Torus mit Weyl-Gruppe $G:=\mathrm{GL}_{3}\left(\mathbb{F}_{2}\right)$; dieser läßt sich so wählen, daß $H^{*}\left(B G_{2}\right) \rightarrow H^{*}\left(B C_{2}^{3}\right)$ mit der Inklusion der $G$-Invarianten

$$
\mathbb{F}_{2}\left[x_{1}, x_{2}, x_{3}\right]^{G} \subset \mathbb{F}_{2}\left[x_{1}, x_{2}, x_{3}\right] \cong H^{*}\left(\mathbb{R} P^{\infty} \times \mathbb{R} P^{\infty} \times \mathbb{R} P^{\infty}\right)=H^{*}\left(B C_{2}^{3}\right)
$$

identifiziert werden kann (siehe [4], Seite 114). Da $G$ auf $G_{2} / C_{2}^{3}$ operiert, kann man aus dem Steinberg-Idempotent $e_{\mathrm{St}} \in \mathbb{Z}_{(2)} G$ eine Abbildung $\Sigma^{\infty} G_{2} / C_{2}^{3} \rightarrow \Sigma^{\infty} G_{2} / C_{2}^{3}$ konstruieren. Deren Bild - worunter man das Teleskop

$$
e_{\mathrm{St}} \Sigma^{\infty} G_{2} / C_{2}^{3}:=\operatorname{hocolim}\left(\Sigma^{\infty} G_{2} / C_{2}^{3} \stackrel{e_{\mathrm{St}}}{\longrightarrow} \Sigma^{\infty} G_{2} / C_{2}^{3} \stackrel{e_{\mathrm{St}}}{\longrightarrow} \Sigma^{\infty} G_{2} / C_{2}^{3} \stackrel{e_{\mathrm{St}}}{\longrightarrow} \cdots\right)
$$

versteht - realisiert dann eine Version von $A_{1}$.

Dwyer und Wilkerson haben in [17] eine sogenannte 2-kompakte Gruppe $D I(4)$ konstruiert. Diese hat einen 2-Torus $C_{2}^{4} \subset D I(4)$ mit Weyl-Gruppe $\mathrm{GL}_{4}\left(\mathbb{F}_{2}\right)$. Dieselbe Argumentation wie oben sollte dann zeigen, daß $e_{\mathrm{St}} \Sigma^{\infty} D I(4) / C_{2}^{4}$ eine Version 
von $A_{2}$ realisiert. Der Autor weiß aber zuwenig über $p$-kompakte Gruppen, als daß er sich hierfür verbürgen könnte.

Wir bemerken noch, daß die $A_{n}$ für den chromatischen Zugang zur Homotopietheorie von Bedeutung sind (siehe [40]).

$B_{n}=\frac{1}{2} A_{n}$ für $n=1,2$. [36] zufolge, ist $B_{n}:=A_{n} / Q_{n} A_{n}$ für geeignete $A_{n}$ ein $A$-Modul. Aufgrund der kurzen exakten Sequenzen $\Sigma^{\left|Q_{n}\right|} B_{n} \longmapsto A_{n} \rightarrow B_{n}$ bezeichnet man $B_{n}$ manchmal als $\frac{1}{2} A_{n}$. Die Abbildungen 3.5 und 3.6 zeigen die Kohomologie der $\Sigma^{\left|Q_{1}\right|} B_{1}$ und $\Sigma^{\left|Q_{2}\right|} B_{2}$, die zu den oben betrachteten $A_{1}$ und $A_{2}$ gehören. Man sieht, daß $\operatorname{Ext}_{A}^{3, t}\left(B_{1}, \mathbb{F}_{2}\right)=0$ für $t \leq 9$ und $\operatorname{Ext}_{A}^{3, t}\left(B_{2}, \mathbb{F}_{2}\right)=0$ für $t \leq 20$. Wegen $D_{B_{1}}=3$ und $D_{B_{2}}=16$, sind also auch $B_{1}$ und $B_{2}$ realisierbar. 
3 Weitere Anwendungen

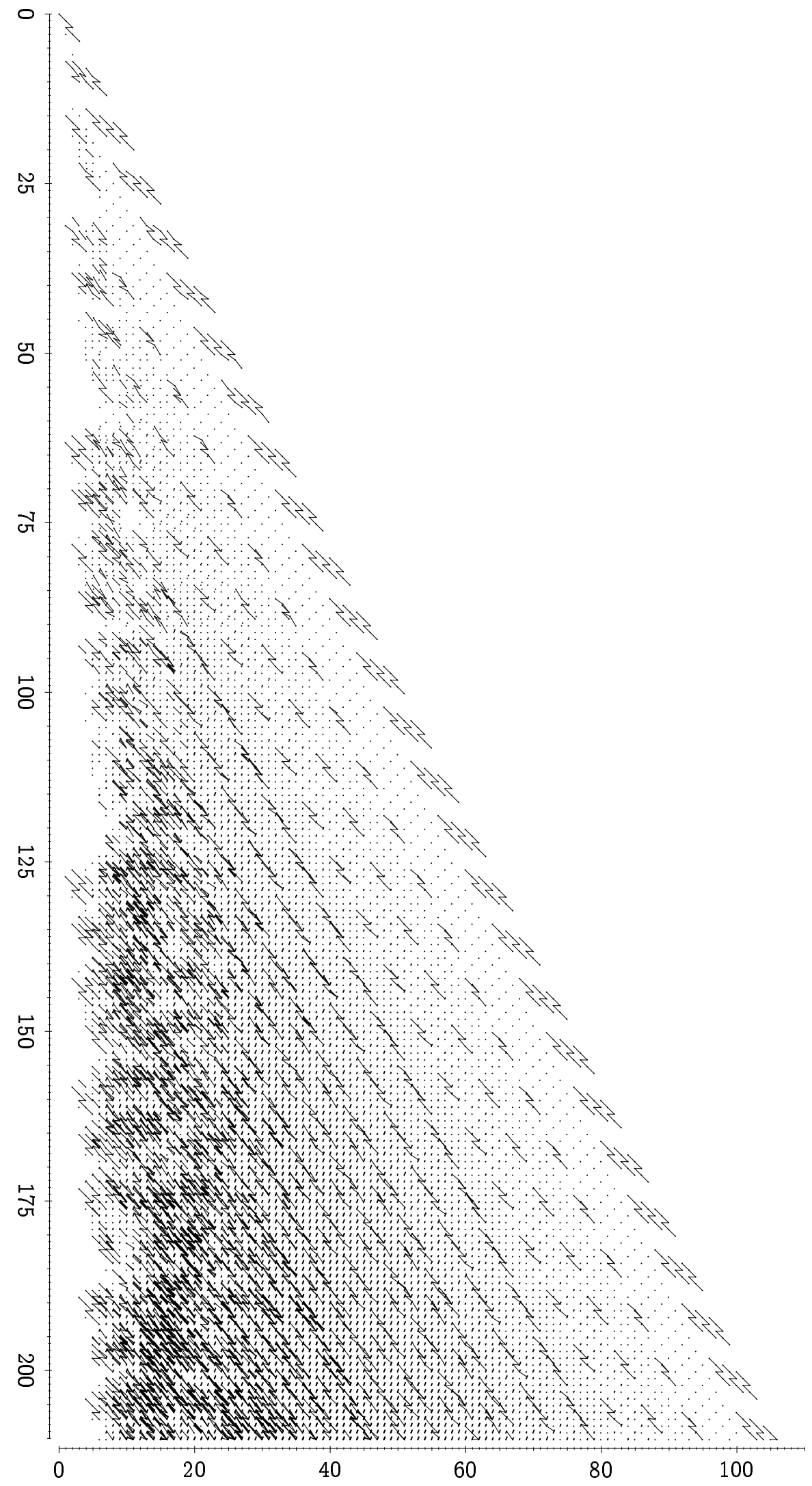

Abbildung 3.1: $\operatorname{Ext}_{A}^{*, *}\left(A_{0}, \mathbb{F}_{2}\right)$ 


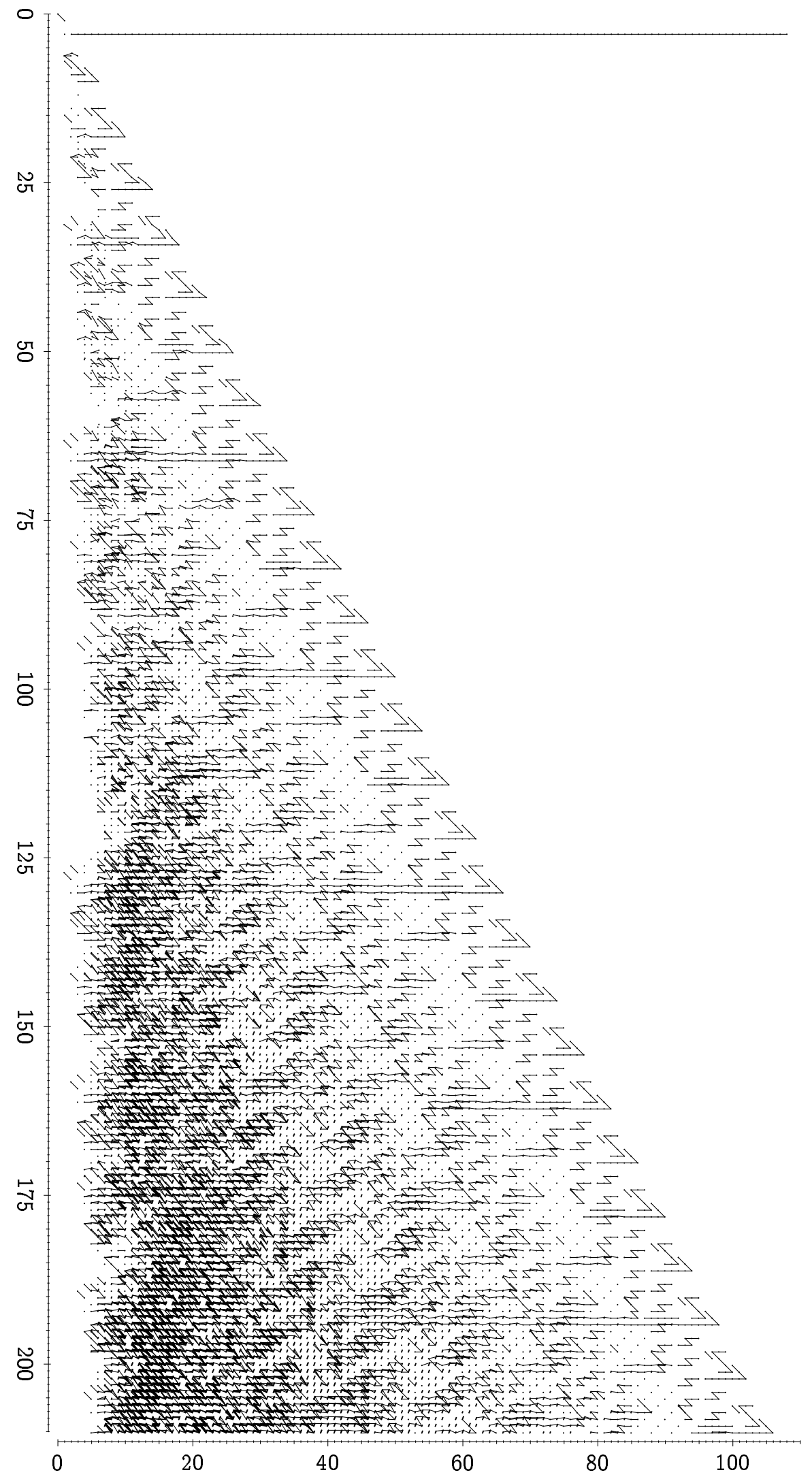

Abbildung 3.2: $\operatorname{Ext}_{A}^{* * *}\left(F, \mathbb{F}_{2}\right)$ 
3 Weitere Anwendungen

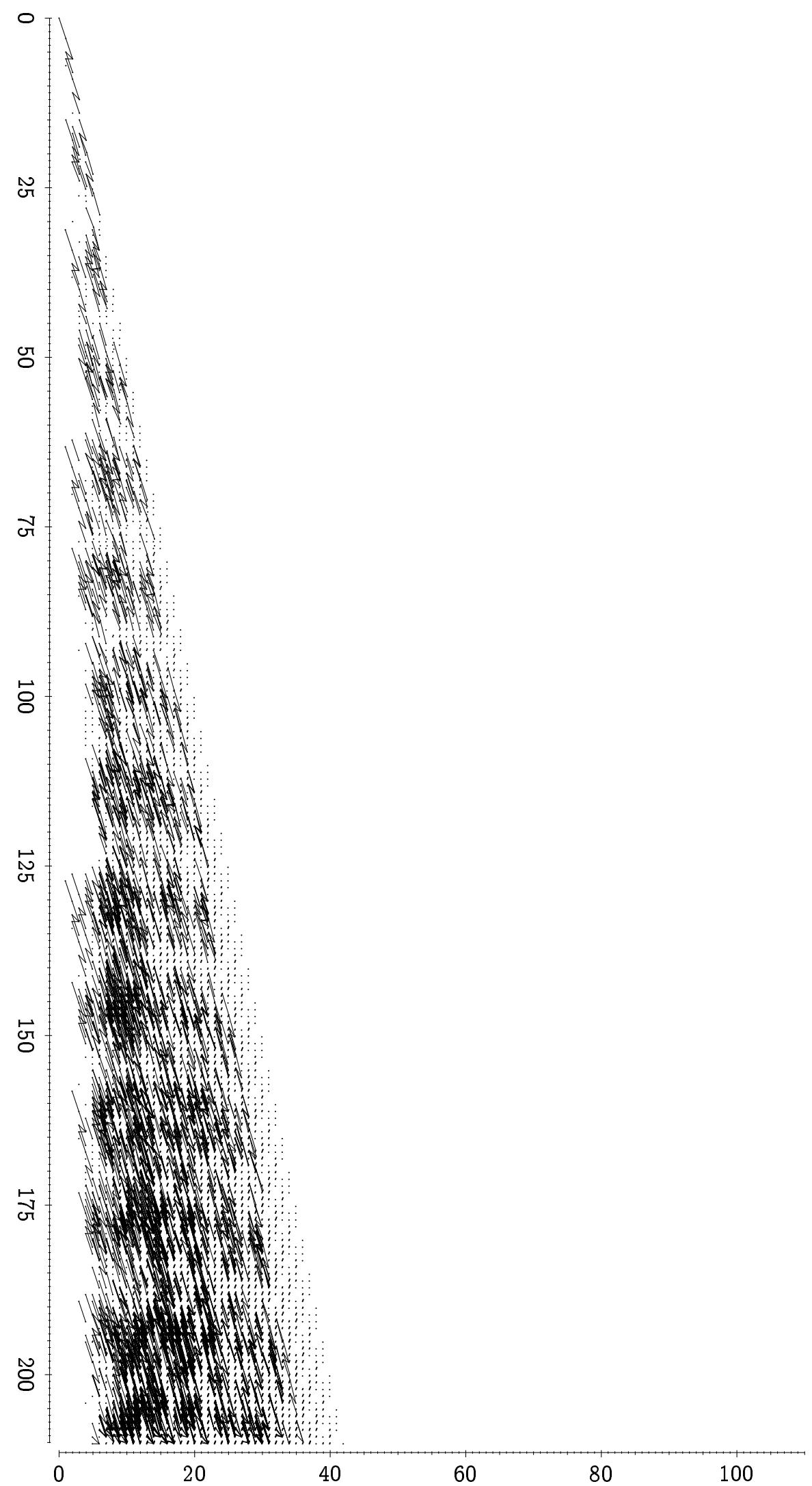

Abbildung 3.3: $\operatorname{Ext}_{A}^{* * *}\left(A_{1}, \mathbb{F}_{2}\right)$ 
$3.1 \operatorname{Ext}_{A}(M, N)$ für kleine $M$ und $N$

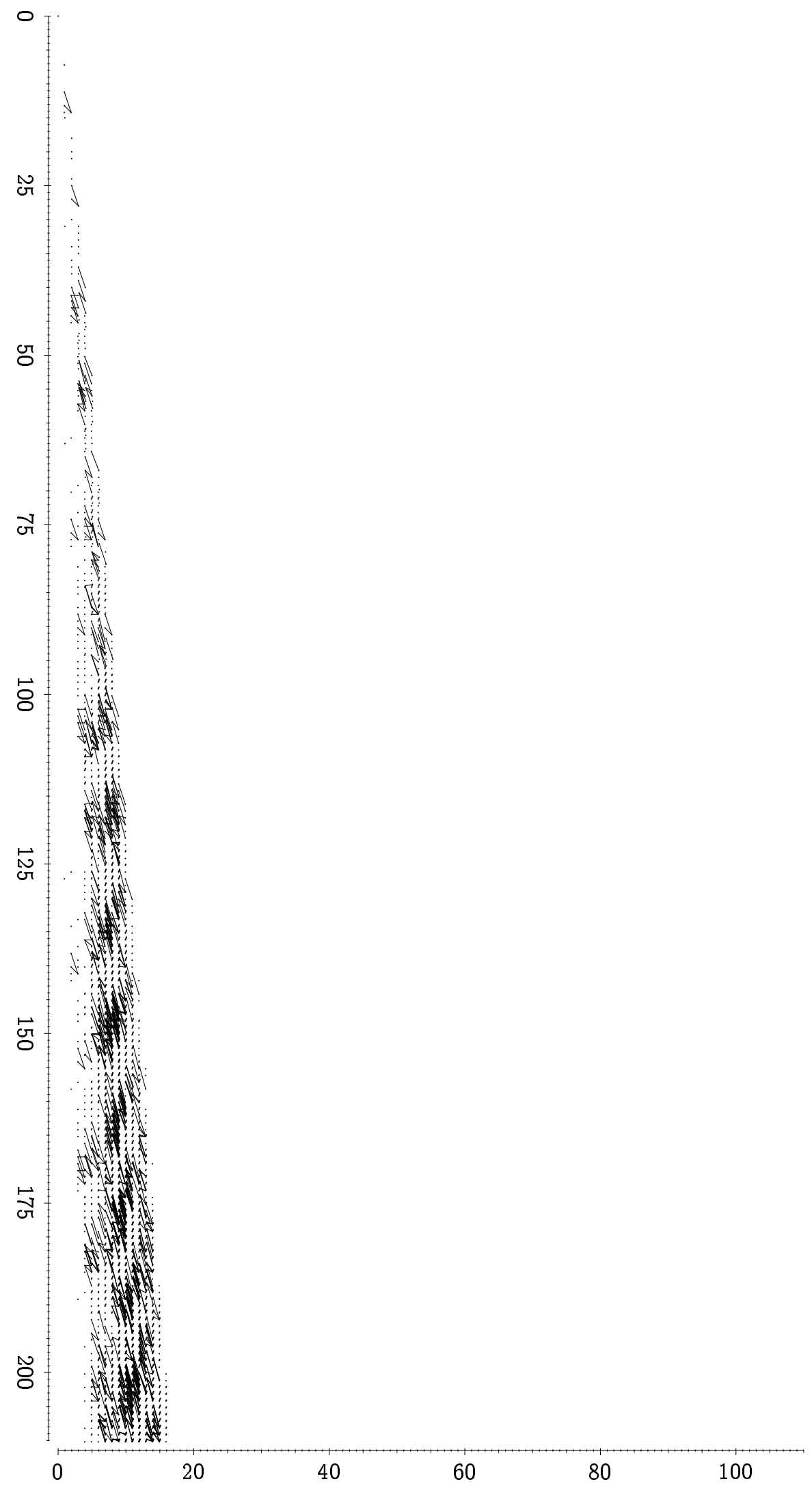

Abbildung 3.4: $\operatorname{Ext}_{A}^{*, *}\left(A_{2}, \mathbb{F}_{2}\right)$ 
3 Weitere Anwendungen

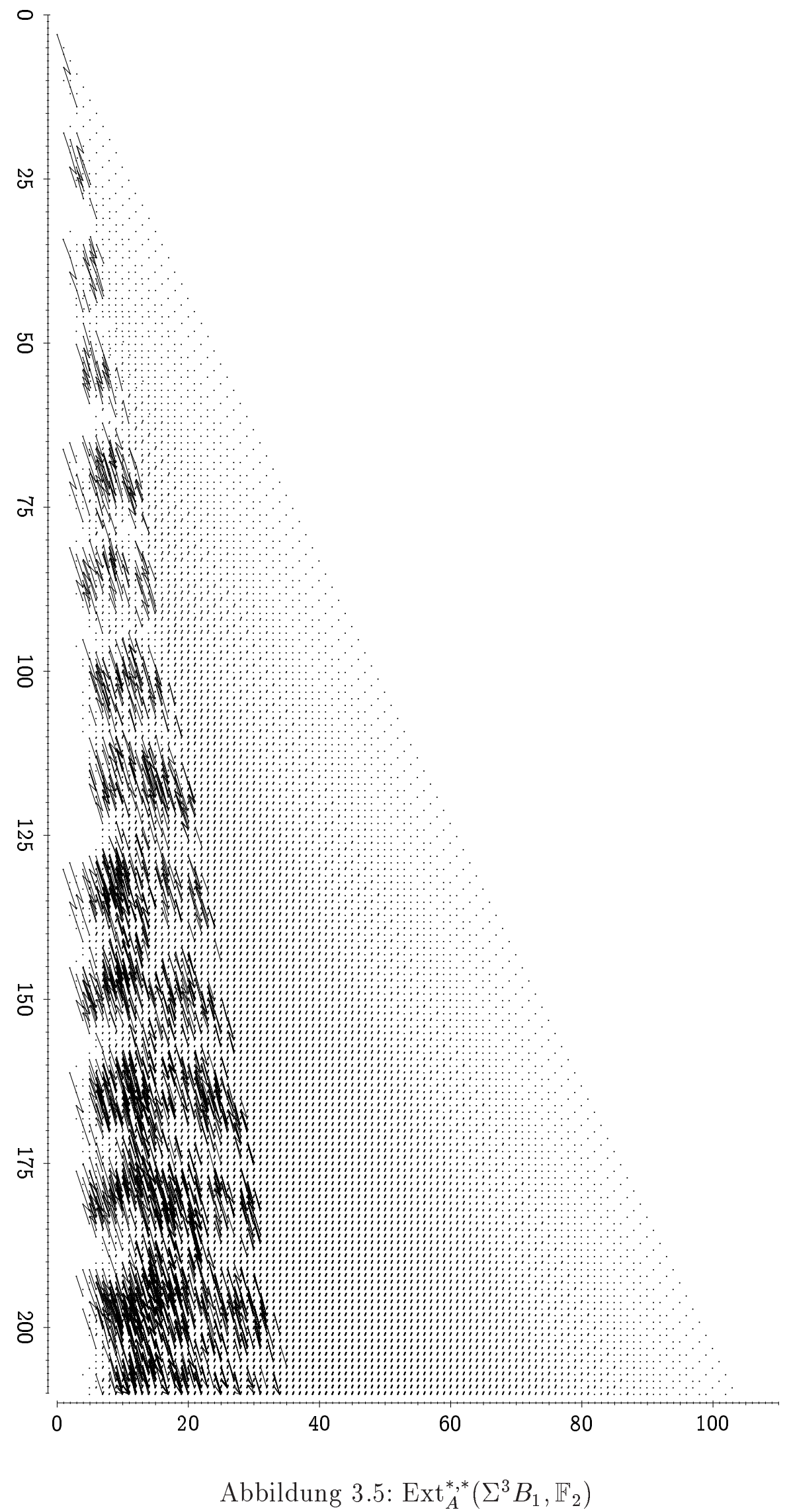


$3.1 \operatorname{Ext}_{A}(M, N)$ für kleine $M$ und $N$

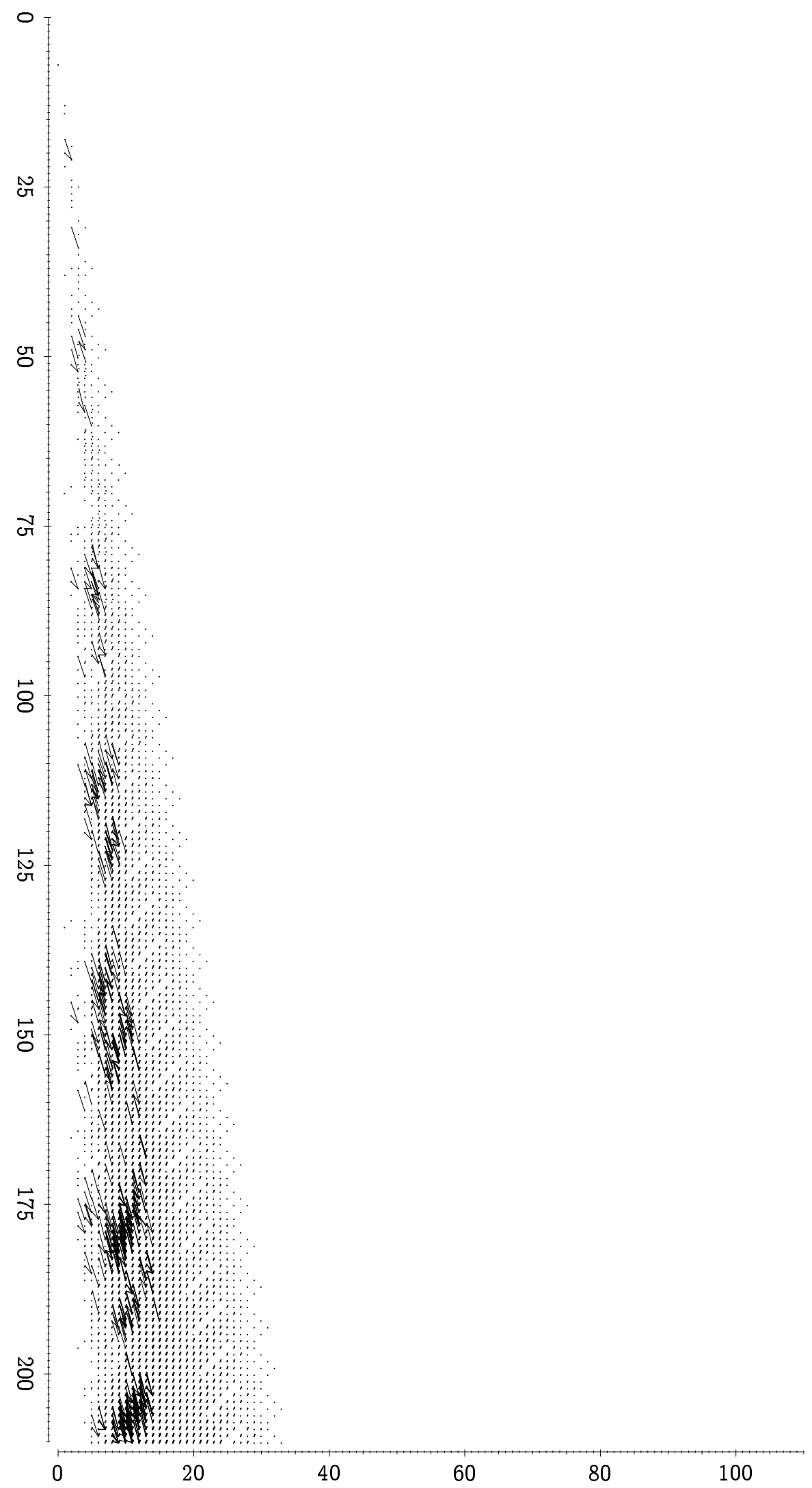

Abbildung 3.6: $\operatorname{Ext}_{A}^{*, *}\left(\Sigma^{7} B_{2}, \mathbb{F}_{2}\right)$ 


\subsection{Wurzelinvarianten}

Bei der im Titel erwähnten Invarianten handelt es sich um eine Konstruktion, die zuerst von Mahowald in [27] betrachtet worden ist und deshalb auch MahowaldInvariante genannt wird. Wir möchten hier die topologische Herkunft und Signifikanz nicht diskutieren; der Leser kann sich darüber zB. aus [13], [10] oder [28] informieren. Stattdessen möchten wir zeigen, wie man mit der Methode des vorigen Abschnitts Wurzeln effektiv berechnen kann.

Sei dazu $P=H^{*}\left(\mathbb{R} P^{\infty} ; \mathbb{F}_{2}\right)$. Bekanntlich ist $P=\mathbb{F}_{2}[x]$ mit $|x|=1$ und $\mathrm{Sq}^{k} x^{n}=$ $\left(\begin{array}{l}n \\ k\end{array}\right) x^{n+k}$. Diese Formel macht auch für negatives $n$ Sinn und definiert eine $A$-Modulstruktur auf dem Lin-Modul $L=\mathbb{F}_{2}\left[x, x^{-1}\right]$. Wie man sich leicht überlegt, ist die Abbildung $\eta: L \rightarrow \Sigma^{-1} \mathbb{F}_{2}$ mit $x^{-1} \mapsto 1 A$-linear. Erstaunlicherweise induziert diese einen Isomorphismus in $\operatorname{Ext}_{A}\left(-, \mathbb{F}_{2}\right)$ :

Satz 3.2.1 ([24]). $\eta^{*}: \operatorname{Ext}_{A}^{s, t}\left(\mathbb{F}_{2}, \mathbb{F}_{2}\right) \rightarrow \operatorname{Ext}_{A}^{s, t+1}\left(L, \mathbb{F}_{2}\right)$ ist ein Isomorphismus.

Man betrachtet nun die Filtrierung von $L$ durch die Untermoduln $L_{k} \subset L$ mit $L_{k}=\mathbb{F}_{2}\left\{x^{n}: n \geq k\right\}$. Sinnvollerweise setzt man $L_{-\infty}=L$. Offenbar ist $L_{k+1} \subset L_{k}$ und $L_{k} / L_{k+1}=\mathbb{F}_{2}\left\{x^{k}\right\} \cong \Sigma^{k} \mathbb{F}_{2}$. Setze $\eta_{k}=\left.\eta\right|_{L_{k}}: L_{k} \rightarrow \Sigma^{-1} \mathbb{F}_{2}$. Sei $0 \neq x \in$ $\operatorname{Ext}_{A}^{s, t}\left(\Sigma^{-1} \mathbb{F}_{2}, \mathbb{F}_{2}\right)$ gegeben. Da $\eta=\eta_{-\infty}$ in Ext ein Isomorphismus ist, ist $\eta_{k}^{*}(x) \neq 0$ für hinreichend kleine $k$. Andererseits ist offenbar $\eta_{k}=0$ für $k \geq 0$, sodaß es ein maximales $k<0$ mit $\eta_{k}^{*}(x) \neq 0$ gibt. Man betrachte zu diesem $k$ das folgende Diagramm, in dem die obere Zeile eine kurze exakte Sequenz ist:

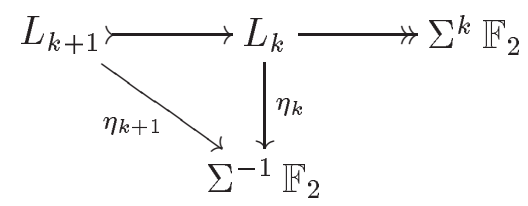

Dieses induziert

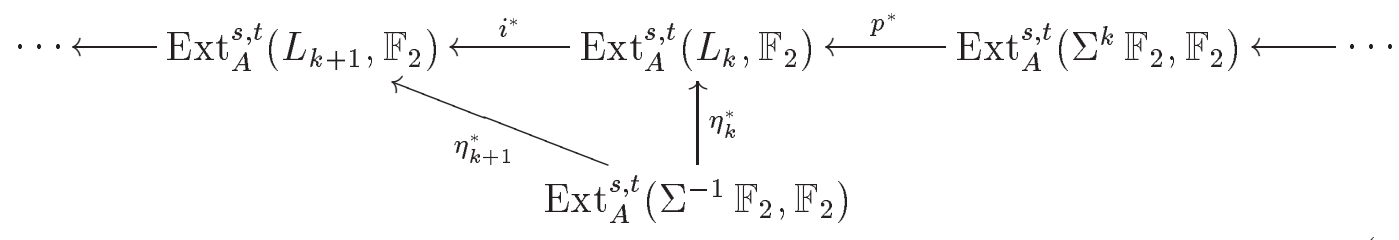

Da nun $i^{*}\left(\eta_{k}^{*}(x)\right)=\eta_{k+1}^{*}(x)=0$, ist $p^{*-1}\left(\eta_{k}^{*}(x)\right)$ nicht leer.

Definition 3.2.2. $R(x):=p^{*-1}\left(\eta_{k}^{*}(x)\right) \subset \operatorname{Ext}_{A}^{s, t+k}\left(\mathbb{F}_{2}, \mathbb{F}_{2}\right)$ heißt Wurzel von $x$.

Man beachte, daß $R(x)$ kein einzelnes Element, sondern eine ganze Nebenklasse des Bildes von $\operatorname{Ext}_{A}^{s-1, t}\left(L_{k+1}, \mathbb{F}_{2}\right) \rightarrow \operatorname{Ext}_{A}^{s, t}\left(\Sigma^{k} \mathbb{F}_{2}, \mathbb{F}_{2}\right)$ bezeichnet.

Zur Berechnung der Wurzeln aus einer minimalen Auflösung $C_{*}$ kann man die Definition ganz direkt umsetzen. Man bildet zunächst das Diagramm

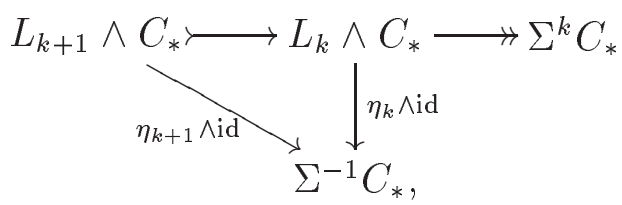




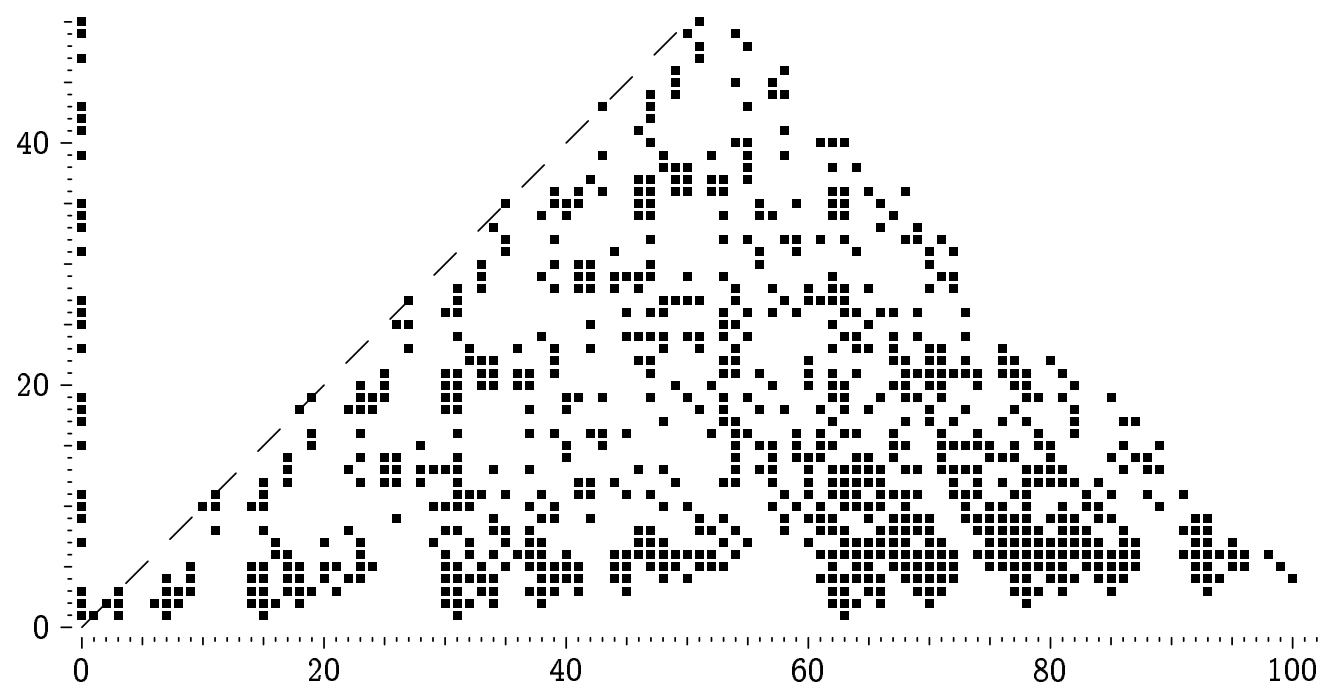

Abbildung 3.7: Bild zur Bredon-Löffler-Vermutung. Für alle $x \in \operatorname{Ext}^{s, n+s}\left(\mathbb{F}_{2}, \mathbb{F}_{2}\right)$, für die von uns die Wurzel $R(x) \subset \mathrm{Ext}^{s, n+s+k(x)}\left(\mathbb{F}_{2}, \mathbb{F}_{2}\right)$ berechnet werden konnte, ist ein schwarzes Kästchen mit Koordinaten $(n, k(x)-n)$ eingezeichnet. Man sieht, daß in allen positiven Dimensionen $n>0$ jeweils $k \leq 2 n$ gilt, wie es die algebraische Bredon-Löffler-Vermutung vorhersagt.

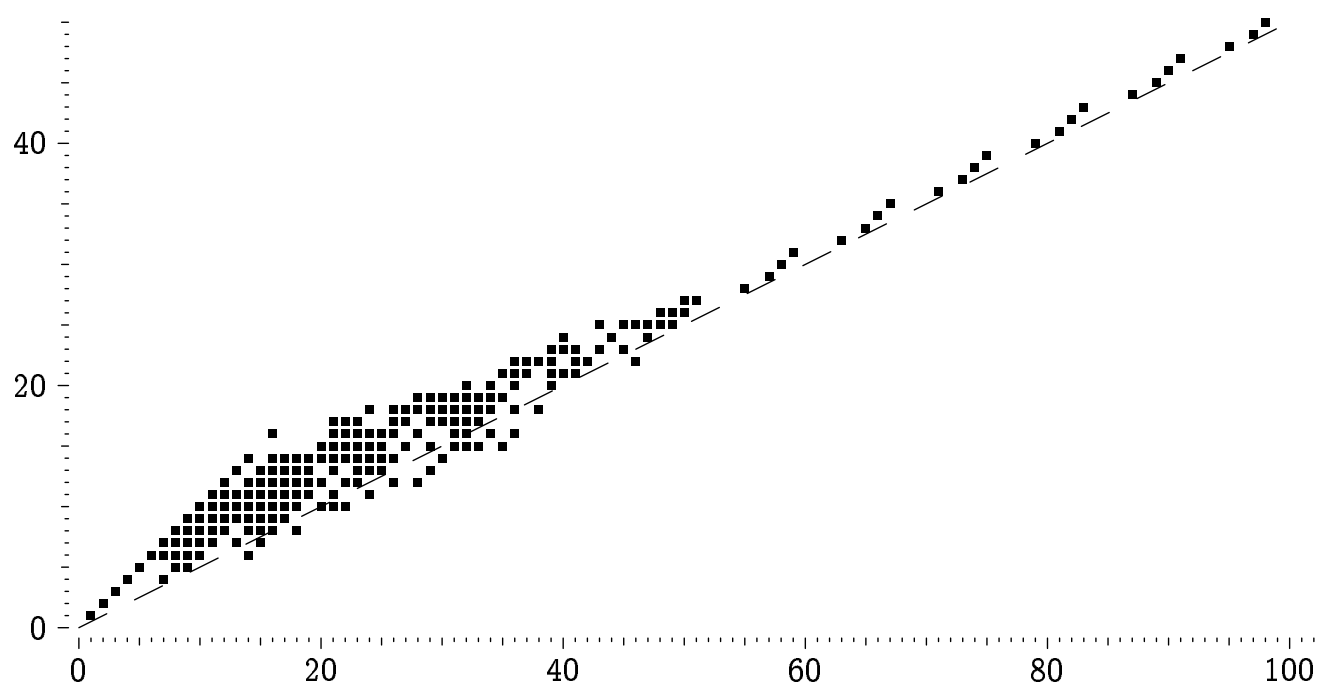

Abbildung 3.8: Bild zur starken Bredon-Löffler-Vermutung. Hier wurde für jedes $R(x) \subset \operatorname{Ext}^{s, n+s+k(x)}\left(\mathbb{F}_{2}, \mathbb{F}_{2}\right)$ ein Kästchen im Feld $(k(x)-n, s)$ geschwärzt. Die starke Bredon-Löffler-Vermutung sagt voraus, daß alle solche Kästchen oberhalb einer Graden mit Steigung 1/2 liegen. Unsere Resultate scheinen dies nicht zu bestätigen. 
bei dem wir stillschweigend $\Sigma^{j} \mathbb{F}_{2} \wedge C_{*}$ mit $\Sigma^{j} C_{*}$ identifiziert haben. Dieses Diagramm ist eine Realisierung von 3.1, in der die Ecken durch partielle Auflösungen und die Pfeile durch zugehörige Kettenabbildungen ersetzt wurden. Das zu 3.1 gehörige Diagramm der Kohomologiegruppen 3.2 kann man also berechnen, indem man $\operatorname{Hom}_{A}\left(-, \mathbb{F}_{2}\right)$ auf 3.3 anwendet.

Um Verwechslungen mit dem Graduierungsstern zu vermeiden, bezeichnen wir im folgenden das Vektorraumdual von $M$ mit $M^{\dagger}$. Wir benutzen den Isomorphismus $\operatorname{Hom}_{A}\left(M \wedge C_{s}, \mathbb{F}_{2}\right)=M^{\dagger} \otimes_{\mathbb{F}_{2}} \operatorname{Ext}_{A}^{s}\left(\mathbb{F}_{2}, \mathbb{F}_{2}\right)$ aus Bemerkung 3.1.4, und können das aus 3.3 entstehende Diagramm von Kettenkomplexen mit

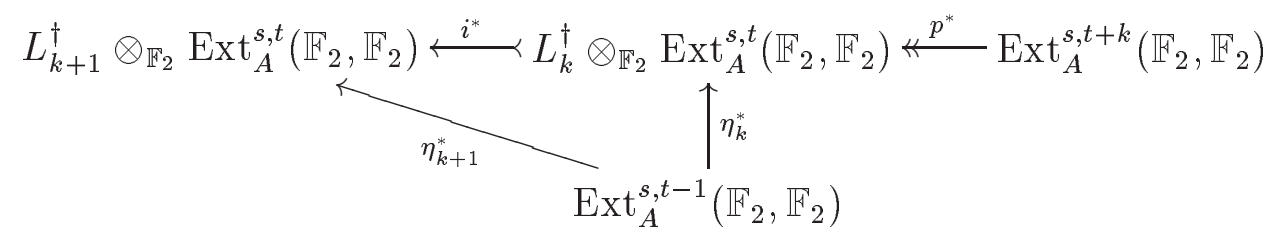

identifizieren. Die auftretenden $L_{k}^{\dagger} \otimes_{\mathbb{F}_{2}} \operatorname{Ext}_{A}^{s, *}\left(\mathbb{F}_{2}, \mathbb{F}_{2}\right)$ sind dabei allesamt Quotientenkomplexe von $L^{\dagger} \otimes_{\mathbb{F}_{2}} \operatorname{Ext}_{A}^{s, *}\left(\mathbb{F}_{2}, \mathbb{F}_{2}\right)$, sodaß man im Grunde nur diesen auf dem Rechner realisieren muß, und dies geht genau wie in Abschnitt 3.1 beschrieben. Unsere Implementierung war in der Lage, innerhalb eines Tages alle Wurzeln, die im Bereich $\operatorname{Ext}_{A}^{s, t}\left(\mathbb{F}_{2}, \mathbb{F}_{2}\right)$ mit $t-s \leq 210$ liegen, zu berechnen. Die Ergebnisse findet man unter [38].

Es ist bekannt, daß die Wurzel eines $x \in \operatorname{Ext}_{A}^{s, n+s}\left(\mathbb{F}_{2}, \mathbb{F}_{2}\right)$ immer mindestens den doppelten internen Grad $2 n+2 s$ hat. Damit ist insbesondere auch die topologische Dimension der Wurzel mindestens $2 n+2 s-s$, also $\geq 2 n$. Es wird vermutet, daß diese aber nie größer als $3 n$ sein kann:

Vermutung 3.2.3 (algebraische Bredon-Löffler-Vermutung, [10], [13]). Sei $n>0$ und $0 \neq x \in \operatorname{Ext}_{A}^{s, n+s}\left(\mathbb{F}_{2}, \mathbb{F}_{2}\right)$ mit $R(x) \subset \operatorname{Ext}_{A}^{s, n+s+k}\left(\mathbb{F}_{2}, \mathbb{F}_{2}\right)$. Dann ist $k \leq 2 n$.

Die Abbildung 3.7 zeigt, daß die von uns berechneten Wurzeln sich an diese Regel halten. Dagegen scheint uns die folgende, von Bruner in [13] und [14] betrachtete Verschärfung im Lichte von Abbildung 3.8 etwas fragwürdig:

Vermutung 3.2.4 (starke algebraische Bredon-Löffler-Vermutung, [13]). Es gibt eine Konstante $C$, sodaß für $n>0$ und $0 \neq x \in \operatorname{Ext}_{A}^{s, n+s}\left(\mathbb{F}_{2}, \mathbb{F}_{2}\right)$ mit $R(x) \subset \operatorname{Ext}_{A}^{s, n+s+k}\left(\mathbb{F}_{2}, \mathbb{F}_{2}\right)$ jeweils $s<(k-n-C) / 2$ gilt.

Ursprünglich wurde dies in [13] für $C=0$ vermutet, bevor der Ausreißer $r_{1} \in$ $R\left(h_{2}^{2} g\right)$ mit Koordinaten $(s, k)=(6,14)$ bekannt war (siehe [14]). Uns scheint, daß die Häufung solcher Ausreißer für $s \leq 20$ zu einer skeptischeren Betrachtung einlädt.

\subsection{Die Frobeniusoperation}

Da die duale Steenrod-Algebra $A_{*}$ eine (graduiert-) kommutative Hopf-Algebra über dem Primkörper $\mathbb{F}_{p}$ ist, kann man die Frobeniusabbildung $F: A_{t} \rightarrow A_{p t}, z \mapsto z^{p}$, betrachten. 
Lemma 3.3.1. $F: A_{*} \rightarrow A_{p *}$ ist ein Homomorphismus von Hopf-Algebren.

Der duale Homomorphismus $F^{\dagger}: A \rightarrow A$ ist auf Milnorschen Basiselementen wie folgt gegeben:

$$
\begin{aligned}
F^{\dagger}(Q(\varepsilon)) & = \begin{cases}1 & \text { falls } Q(\varepsilon)=1, \\
0 & \text { sonst },\end{cases} \\
F^{\dagger}\left(P\left(r_{1}, \ldots, r_{n}\right)\right) & = \begin{cases}P\left(\frac{r_{1}}{p}, \ldots, \frac{r_{n}}{p}\right) & \text { falls alle } r_{i} \equiv 0 \bmod p, \\
0 & \text { sonst. }\end{cases}
\end{aligned}
$$

Man definiert einen Funktor $F^{\dagger}: A$-Mod $\rightarrow A$-Mod durch

$$
\left(F^{\dagger} M\right)_{t}:= \begin{cases}M_{t / p} & \text { falls } t \equiv 0 \bmod p \\ 0 & \text { sonst }\end{cases}
$$

wobei die $A$-Operation $A_{t} \otimes F_{k}^{\dagger} \rightarrow F_{t+k}^{\dagger}$ nur für $t \equiv 0 \bmod p$ und $k \equiv 0 \bmod p$ nicht null sei. In diesem Fall sei sie durch

$$
A_{p t} \otimes F^{\dagger} M_{p k} \stackrel{F^{\dagger} \otimes \mathrm{id}}{\longrightarrow} A_{t} \otimes M_{k} \stackrel{\nu_{M}}{\longrightarrow} M_{t+k} \cong F_{p t+p k}^{\dagger}
$$

gegeben. Man vergleiche [29], Ch. 15.3.

$F^{\dagger}$ ist offenbar ein exakter Funktor. Er induziert damit eine natürliche Transformation

$$
F^{\dagger}: \operatorname{Ext}_{A}^{s, t}(M, N) \rightarrow \operatorname{Ext}_{A}^{s, t}\left(F^{\dagger} M, F^{\dagger} N\right),
$$

die man folgendermaßen berechnet: sei dazu $C_{*}$ eine $A$-freie Auflösung von $M$ und $\tilde{C}_{*}$ eine $A$-freie Auflösung von $F^{\dagger} M$. Da $F^{\dagger}$ exakt ist, kann man die Homologie $H_{s}\left(F^{\dagger} C_{*}\right)$ mit $F^{\dagger} H_{s}\left(C_{*}\right)$ identifizieren. Es ist also

$$
H_{s}\left(F^{\dagger} C_{*}\right)= \begin{cases}F^{\dagger} M & s=0 \\ 0 & s \neq 0\end{cases}
$$

sodaß $F^{\dagger} C_{*}$ eine (nicht-freie) Auflösung von $F^{\dagger} M$ ist. Es gibt mithin eine bis auf Homotopie eindeutig bestimmte Vergleichsabbildung $\phi_{*}: \tilde{C}_{*} \rightarrow F^{\dagger} C_{*}$. Ist nun $\alpha$ : $C_{s} \rightarrow N$ ein Kozykel, der eine Klasse $[\alpha] \in \operatorname{Ext}_{A}^{s, t}(M, N)$ repräsentiert, so setzt man $F^{\dagger}([\alpha])=\left[F^{\dagger} \alpha \circ \phi_{s}\right] \in \operatorname{Ext}_{A}^{s, t}\left(F^{\dagger} M, F^{\dagger} N\right)$.

Da $F^{\dagger} \Sigma^{k} \mathbb{F}_{p}=\Sigma^{p k} \mathbb{F}_{p}$ ist, erhält man als Spezialfall eine Transformation

$$
\operatorname{Ext}_{A}^{s, t}\left(\mathbb{F}_{p}, \mathbb{F}_{p}\right) \rightarrow \operatorname{Ext}_{A}^{s, p t}\left(\mathbb{F}_{p}, \mathbb{F}_{p}\right)
$$

Hier kann man oben $\tilde{C}_{*}=C_{*}$ setzen, sodaß nur eine Vergleichsabbildung $\phi_{*}: C_{*} \rightarrow$ $F^{\dagger} C_{*}$ berechnet werden muß. Auf dem Computer ist dies überraschend einfach: einerseits ist nämlich die rechte Seite deutlich kleiner als $C_{*}$ selbst, und andererseits kann man die Theorie aus Kapitel 2 auch für $F^{\dagger} C_{*}$ benutzen. Schließlich wurde dort nur die Exaktheit gewisser Sequenzen benutzt und diese bleibt bei Anwendung von $F^{\dagger}$ erhalten. Für $p=2$ dauerte die Berechnung im ganzen verfügbaren Bereich $2 t-s \leq 210$ 


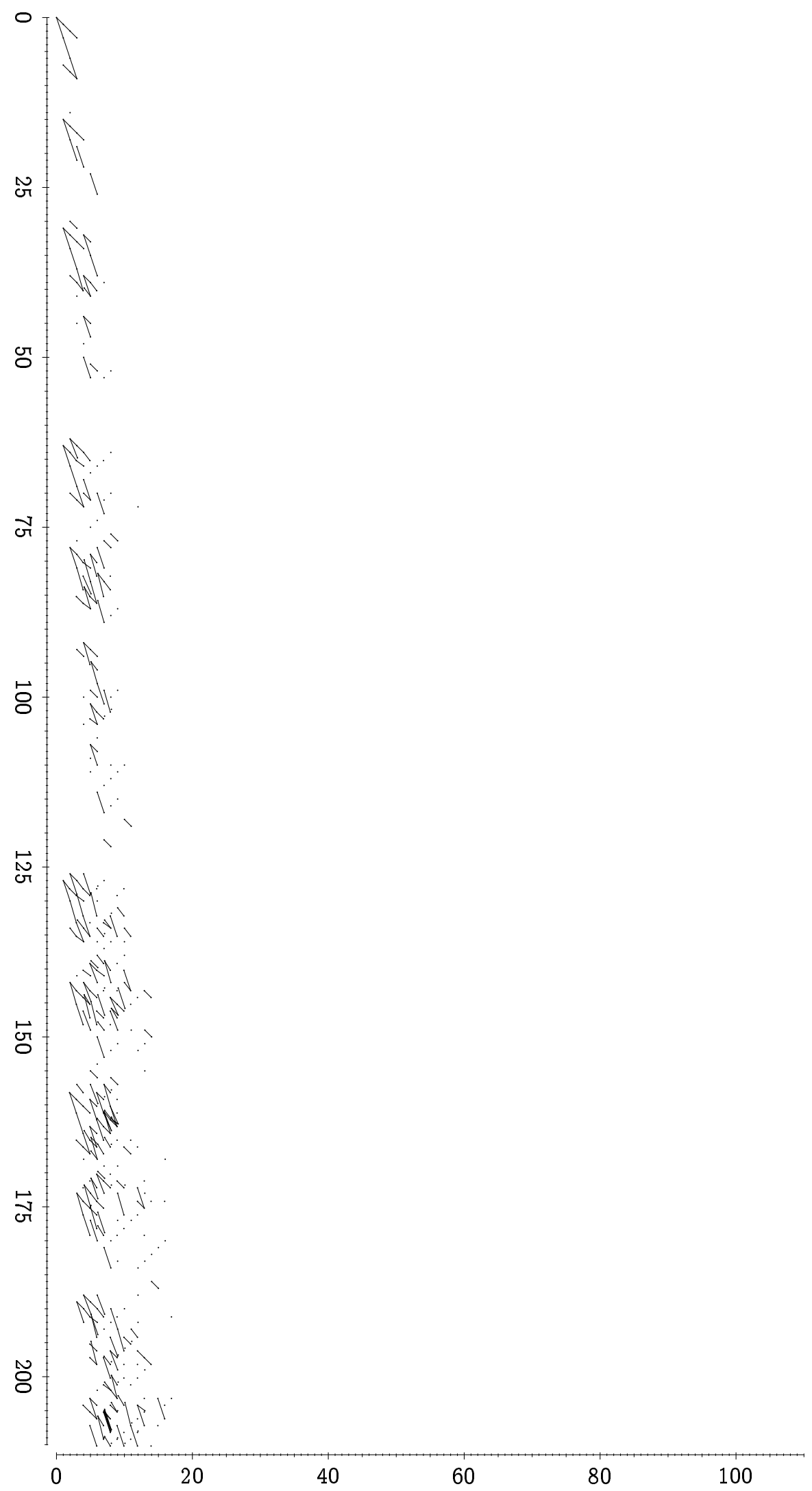

Abbildung 3.9: Bild von $\operatorname{Sq}^{0}: \operatorname{Ext}_{A}^{*, *}\left(\mathbb{F}_{2}, \mathbb{F}_{2}\right) \rightarrow \operatorname{Ext}_{A}^{*, 2 *}\left(\mathbb{F}_{2}, \mathbb{F}_{2}\right)$ 
nur wenige Stunden. Detaillierte Resultate findet man wiederum in [38]. Wir geben hier nur in Abbildung 3.9 das Bild von $F^{\dagger}: \operatorname{Ext}_{A}^{*, *}\left(\mathbb{F}_{2}, \mathbb{F}_{2}\right) \rightarrow \operatorname{Ext}_{A}^{*, 2 *}\left(\mathbb{F}_{2}, \mathbb{F}_{2}\right)$ an.

Tatsächlich ist $F^{\dagger}$ nur die nullte einer ganzen Familie interessanter Operationen: so wie die Steenrod-Algebra auf der Kohomologie topologischer Räume operiert, operiert auch eine Variante auf der Kohomologie kokommutativer Hopf-Algebren über $\mathbb{F}_{p}$. Für $p=2$ und $i \in \mathbb{N}_{0}$ zB. erhält man Abbildungen

$$
\mathrm{Sq}^{i}: \operatorname{Ext}_{A}^{s, t}\left(\mathbb{F}_{2}, \mathbb{F}_{2}\right) \rightarrow \operatorname{Ext}_{A}^{s+i, 2 t}\left(\mathbb{F}_{2}, \mathbb{F}_{2}\right)
$$

und für $p>2$

$$
\begin{aligned}
P^{i}: \operatorname{Ext}_{A}^{s, t}\left(\mathbb{F}_{p}, \mathbb{F}_{p}\right) & \rightarrow \operatorname{Ext}_{A}^{s+i, p t}\left(\mathbb{F}_{p}, \mathbb{F}_{p}\right) \\
\beta P^{i}: \operatorname{Ext}_{A}^{s, t}\left(\mathbb{F}_{p}, \mathbb{F}_{p}\right) & \rightarrow \operatorname{Ext}_{A}^{s+i+1, p t}\left(\mathbb{F}_{p}, \mathbb{F}_{p}\right)
\end{aligned}
$$

Diese Abbildungen sind

- rein algebraisch definiert (siehe zB. [31] oder [32] Th. 9.10),

- für die Berechnung der Differentiale in der Adams-Spektralreihe von Bedeutung (siehe Bruners Beiträge in [9]),

- und sie verhalten sich ähnlich wie die Operation der $\mathrm{Sq}^{i} \in A$ (bzw. $P^{i}, Q_{0} P^{i} \in$ $A$ falls $p>2$ ) auf der Kohomologie von Räumen.

Leider sind die $P^{i}$ und $\mathrm{Sq}^{i}$ für $i>0$ praktisch unberechenbar: außer in zwei Grenzfällen ist kein Verfahren bekannt, das diese Operationen mit vertretbarem Aufwand aus einer Auflösung berechnen könnte (siehe Bruners Diskussion in [15]).

Die zwei genannten Ausnahmen sind folgende: zum einen ist (für $p=2$ ) $\mathrm{Sq}^{s} x=$ $x^{2}$ falls $x$ den homologischen Grad $s$ hat, und eine entsprechende Formel gilt auch für $p>2$; zum anderen hat man

Satz 3.3.2. Es ist $F^{\dagger}=P^{0}: \operatorname{Ext}_{A}^{s, t}\left(\mathbb{F}_{p}, \mathbb{F}_{p}\right) \rightarrow \operatorname{Ext}_{A}^{s, p t}\left(\mathbb{F}_{p}, \mathbb{F}_{p}\right)$. Für $p=2$ lese man $P^{0}$ als $\mathrm{Sq}^{0}$.

Beweis. Dies ist Prop. 11.10 aus [31]. Dort wird gezeigt, daß man $P^{0}$ auf Repräsentanten in der Kobar-Auflösung der dualen Steenrod-Algebra durch $P^{0}\left(\left[\alpha_{1}|\cdots| \alpha_{s}\right]\right)$ $=\left[\alpha_{1}^{p}|\cdots| \alpha_{s}^{p}\right]$ für $\alpha_{i} \in A_{*}$ berechnen kann. Offenbar ist dies gerade die vom Frobenius $F$ induzierte Abbildung auf der Kohomologie.

Die Kenntnis von $\mathrm{Sq}^{0}$ kann für die Berechnung der höheren $\mathrm{Sq}^{i}$ manchmal hilfreich sein. Für die $\mathrm{Sq}^{i}$ gilt nämlich die Rechenregel $\mathrm{Sq}^{i} \mathrm{Sq}^{0}=\mathrm{Sq}^{0} \mathrm{Sq}^{i}$, sodaß im $\mathrm{Sq}^{0}$ unter den $\mathrm{Sq}^{i}$ abgeschlossen ist. Da im $\mathrm{Sq}^{0}$ nach Abbildung 3.9 sehr klein ist, kommen für die $\mathrm{Sq}^{i} \mathrm{Sq}^{0}(x)$ also immer nur wenige Elemente in Frage.

Wir erwähnen noch, daß $\mathrm{Sq}^{0}$ und die Wurzelinvariante eng miteinander verbunden sind: falls nämlich $\mathrm{Sq}^{0}(x)$ und $R(x)$ dieselbe Dimension haben, so ist $\operatorname{Sq}^{0}(x) \in$ $R(x)[28,2.9]$. 


\subsection{Hurewicz-Abbildung für tmf}

Die im Titel gemeinte Abbildung ist schlicht die natürliche Transformation

$$
\mathrm{H}_{2}: \operatorname{Ext}_{A}^{s, t}\left(\mathbb{F}_{2}, \mathbb{F}_{2}\right) \rightarrow \operatorname{Ext}_{A(2)}^{s, t}\left(\mathbb{F}_{2}, \mathbb{F}_{2}\right) \text {. }
$$

$A(2)$ sei dabei die Hopf-Unteralgebra $B(3,2,1) \subset A$. Die beiden auftretenden ExtGruppen sind bereits in den Abbildungen 2.10 und 2.11 gezeigt worden. Wir stellen hier $\mathrm{H}_{2}$ in Abbildung 3.11 durch sein Bild dar; detailliertere Informationen findet man unter [38]. Wir müssen also nur noch zwei Sachen erklären:

- Wie die Transformation $\mathrm{H}_{2}$ zustande kommt und wie man sie berechnet.

- Welche topologische Bedeutung mit $\mathrm{H}_{2}$ verbunden ist.

Algebraisch betrachtet geht es hier um folgende Konstruktion: uns stehen bereits zwei Auflösungen zur Verfügung, nämlich

- eine minimale $A$-freie Auflösung $C_{*}$ von $\mathbb{F}_{2}$ und

- eine minimale $A(2)$-freie Auflösung $D_{*}$ von $\mathbb{F}_{2}$.

Wir berechnen nun eine $A(2)$-lineare Vergleichsabbildung $\Phi_{*}: D_{*} \rightarrow C_{*}$, die die Identität auf $\mathbb{F}_{2}$ liftet. Ein solches $\Phi_{*}$ ist bis auf Homotopie eindeutig bestimmt. Bei der Berechnung kann man natürlich wiederum die Theorie aus Kapitel 2 zur Vereinfachung der auftretenden Hebungsprobleme in $C_{*}$ benutzen. Hat man $\Phi_{*}$ und ist ein $[\alpha] \in \operatorname{Ext}_{A}^{s, t}\left(\mathbb{F}_{2}, \mathbb{F}_{2}\right)$ durch einen Kozykel $\alpha: C_{s} \rightarrow \Sigma^{t} \mathbb{F}_{2}$ gegeben, so wird $\mathrm{H}_{2}([\alpha]) \in \operatorname{Ext}_{A(2)}^{s, t}\left(\mathbb{F}_{2}, \mathbb{F}_{2}\right)$ durch $\alpha \circ \Phi_{s}: D_{s} \rightarrow \Sigma^{t} \mathbb{F}_{2}$ repräsentiert. $\mathrm{H}_{2}$ kann man also aus $\Phi_{*}$ einfach ablesen. Die Berechnung von $\Phi_{*}$ im gesamten Bereich $t-s \leq 210$ dauerte etwas weniger als zwei Tage.

Topologisch würde man das $\mathrm{H}_{2}$ wie folgt interpretieren: man betrachte allgemeiner

$$
\mathrm{H}_{n}: \operatorname{Ext}_{A}^{s, t}\left(\mathbb{F}_{2}, \mathbb{F}_{2}\right) \rightarrow \operatorname{Ext}_{A(n)}^{s, t}\left(\mathbb{F}_{2}, \mathbb{F}_{2}\right) .
$$

Es gibt einen Isomorphismus (siehe zB. [32, Seite 438]) $\operatorname{Ext}_{A(n)}^{s, t}\left(\mathbb{F}_{2}, \mathbb{F}_{2}\right) \cong \operatorname{Ext}_{A}^{s, t}(A / / A(n)$, $\mathbb{F}_{2}$ ), bei dem $\mathrm{H}_{n}$ mit der von $A / / A(n) \rightarrow \mathbb{F}_{2}$ induzierten Abbildung identifiziert werden kann. Für $n \leq 2$ kann man außerdem $A / / A(n)$ als Kohomologie eines Spektrums schreiben:

$$
\begin{aligned}
H^{*}\left(H \mathbb{F}_{2} ; \mathbb{F}_{2}\right) & =A / / A(-1) & ( & \left.=A \text { weil } A(-1)=\mathbb{F}_{2}\right) \\
H^{*}\left(H \mathbb{Z}_{\mathbb{F}_{2}}\right) & =A / / A(0) & ( & \left.=A / A Q_{0}, \text { siehe Abschnitt } 1.2 .5\right) \\
H^{*}\left(\mathrm{ko} ; \mathbb{F}_{2}\right) & =A / / A(1) & \text { ko } & =\text { zusammenhängende reelle } K \text {-Theorie } \\
H^{*}\left(\mathrm{tmf} ; \mathbb{F}_{2}\right) & =A / / A(2) & \text { tmf } & =\text { Theorie der topologischen Modulformen. }
\end{aligned}
$$

Ist $H^{*}\left(X_{n} ; \mathbb{F}_{2}\right)=A / / A(n)$, so wird zudem $A / / A(n) \rightarrow \mathbb{F}_{2}$ von einer Abbildung $\iota_{n}: S \rightarrow X_{n}$ induziert. Betrachtet man die Adams-Spektralreihen für die Homotopiegruppen von $X_{n}$ bzw. der Sphäre $S$, so erhält man das Diagramm

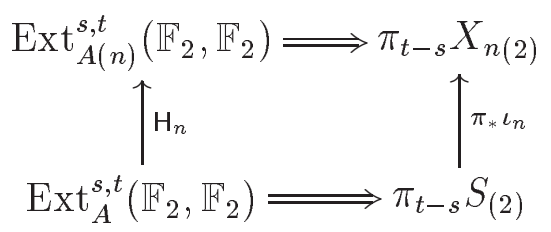




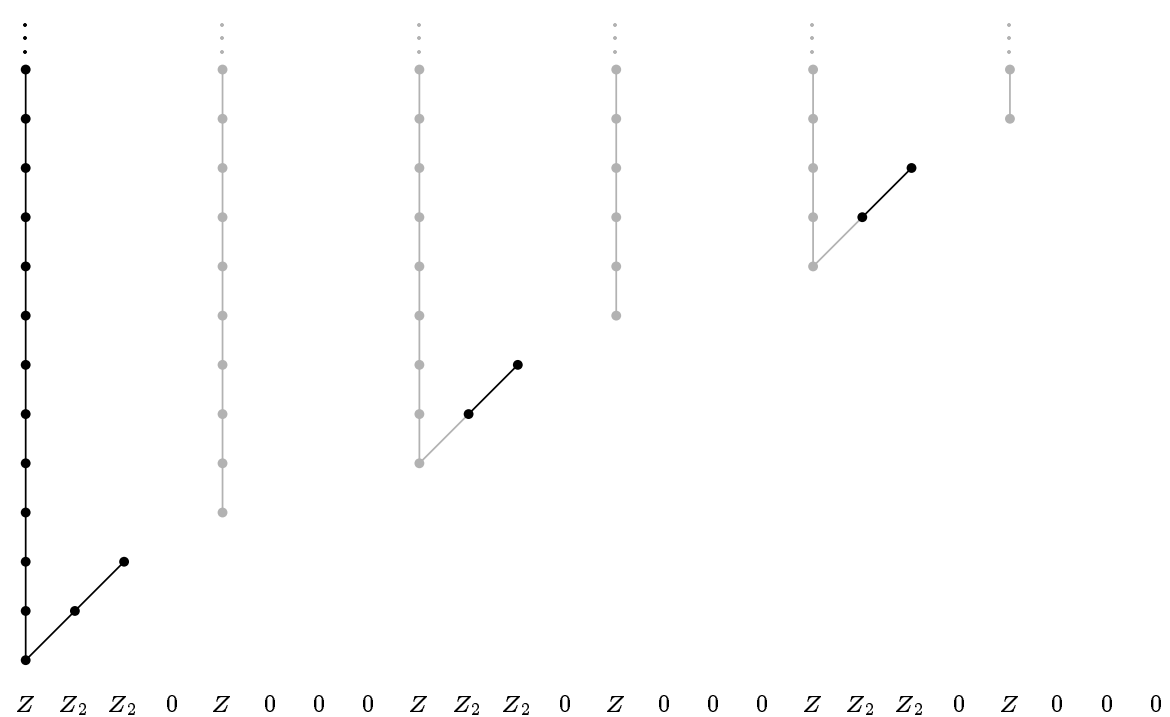

Abbildung 3.10: Ausgangsterm $E_{2}^{s, t}=\operatorname{Ext}_{A}^{s, t}\left(A / / A(1), \mathbb{F}_{2}\right)$ der Adams-Spektralreihe für $\pi_{*} \mathrm{ko}_{(2)}$, welche kollabiert. In schwarz werden die Klassen hervorgehoben, die dem Bild von $\pi_{*} S \rightarrow \pi_{*}$ ko entsprechen.

in dem $\mathrm{H}_{n}$ offenbar zu $\pi_{*} \iota_{n}: \pi_{*} S \rightarrow \pi_{*} X_{n}$ assoziiert ist. Diese nennt man oft auch die $X_{n}$-Hurewicz-Abbildung. Für $n<2$ ist diese bereits völlig verstanden:

$n=-1$. Hier ist $X_{-1}=H \mathbb{F}_{2}$. Die Abbildung $\pi_{*} S \rightarrow \pi_{*} H \mathbb{F}_{2}$ ordnet einem $f \in \pi_{0} S$ den Abbildungsgrad $\operatorname{deg} f \bmod 2$ zu.

$n=0$. Hier ist $X_{0}=H \mathbb{Z}$. Dies ist der klassische Fall, dem die Bezeichnung Hurewicz-Abbildung geschuldet ist: die Abbildung $\iota_{0}: S \rightarrow H \mathbb{Z}$ induziert gerade die Transformation $\pi_{n}(X) \rightarrow H_{n}(X ; \mathbb{Z})$, bei der einem $f: S^{n} \rightarrow X$ das Bild $f_{*}\left(\left[S^{n}\right]\right) \in H_{n}(X ; \mathbb{Z})$ der Fundamentalklasse $\left[S^{n}\right] \in H_{n}\left(S^{n}\right)$ zugeordnet wird. Setzt man hier $X=S^{0}$, so erhält man eine Invariante für Abbildungen zwischen Sphären: eine Klasse $f \in \pi_{0}(S)$ der Dimension Null wird auf ihren Abbildungsgrad $\operatorname{deg} f \in$ $\mathbb{Z} \cong H_{0}\left(S^{0}, \mathbb{Z}\right)$ abgebildet, Klassen anderer Dimension auf Null.

In Ext ergibt sich dasselbe Bild: $\operatorname{Ext}_{A}^{s, t}\left(A / / A(0), \mathbb{F}_{2}\right)$ wurde in Abbildung 1.3 gezeigt. Die Hurewicz-Abbildung $\operatorname{Ext}_{A}^{s, t}\left(\mathbb{F}_{2}, \mathbb{F}_{2}\right) \rightarrow \operatorname{Ext}_{A}^{s, t}\left(A / / A(0), \mathbb{F}_{2}\right)$ ist für $0=t-s$ ein Isomorphismus und entspricht dort dem Abbildungsgrad $\mathbb{Z} \cong \pi_{0}(S) \rightarrow \pi_{0} H \mathbb{Z}=$ $\mathbb{Z}$. Für $t-s \neq 0$ ist sie Null.

$n=1$. Hier ist $X_{1}=$ ko das Spektrum der zusammenhängenden reellen $K$-Theorie. Diese unterscheidet sich von der gewöhnlichen reellen $K$-Theorie KO nur dadurch, daß die Homotopiegruppen in negativen Dimensionen getötet wurden: für KO hat man die bekannten Bott-periodischen Gruppen

\begin{tabular}{r|llllllll}
$k \bmod 8$ & 0 & 1 & 2 & 3 & 4 & 5 & 6 & 7 \\
\hline$\pi_{k}(\mathrm{KO})$ & $\mathbb{Z}$ & $\mathbb{Z}_{2}$ & $\mathbb{Z}_{2}$ & 0 & $\mathbb{Z}$ & 0 & 0 & 0
\end{tabular}


und für ko gilt (nach Konstruktion)

$$
\pi_{k}(\mathrm{ko})= \begin{cases}\pi_{k}(\mathrm{KO}) & k \geq 0 \\ 0 & k<0\end{cases}
$$

Außerdem hat man eine Transformation ko $\rightarrow \mathrm{KO}$, die für nichtnegatives $k$ den Isomorphismus $\pi_{k}(\mathrm{ko}) \rightarrow \pi_{k}(K O)$ induziert.

Daß $H^{*}\left(\mathrm{ko} ; \mathbb{F}_{2}\right)=A / / A(1)$ ist findet man zB. in [26]. In Abbildung 3.10 sieht man zunächst $\operatorname{Ext}_{A}^{*, *}\left(H^{*}(\mathrm{ko}), \mathbb{F}_{2}\right)$, worin diejenigen Klassen in Schwarz hervorgehoben sind, die von $\operatorname{Ext}_{A}^{* * *}\left(\mathbb{F}_{2}, \mathbb{F}_{2}\right)$ herkommen. Neben den Klassen mit Dimension $t-s=0$, die durch den Abbildungsgrad beschrieben werden können, erkennt man die sogenannte $\mu$-Familie von Adams [1] in den Dimensionen $\equiv 1,2 \bmod 8$.

$n=2$. Hier ist $X_{2}=\operatorname{tmf}$ das noch ziemlich mysteriöse Spektrum der topologischen Modulformen. Daß $H^{*}\left(\mathrm{tmf} ; \mathbb{F}_{2}\right)=A / / A(2)$ gilt, ist Theorem 9.2 aus [20]. Dort sind auch die Differentiale der Adams-Spektralreihe

$$
\operatorname{Ext}_{A(2)}^{s, t}\left(\mathbb{F}_{2}, \mathbb{F}_{2}\right) \Rightarrow \pi_{*} \operatorname{tmf}_{(2)}
$$

vollständig berechnet worden.

Was topologische Modulformen sind (oder sein sollen), können wir hier nicht erklären, weil dies den Wissensstand zumindest dieses Autors übersteigt. Unter anderem geht es darum, Witten's Beobachtung aus [47], daß nämlich die $S^{1}$-äquivarianten Indices gewisser hypothetischer Dirac-Operatoren auf freien Schleifenräumen die Fourierdarstellungen von Modulformen sind, in richtiger Allgemeinheit neu zu verstehen (siehe [19]). Wir verweisen den Leser dazu auf [20], [6]. 
3.4 Hurewicz-Abbildung für tmf

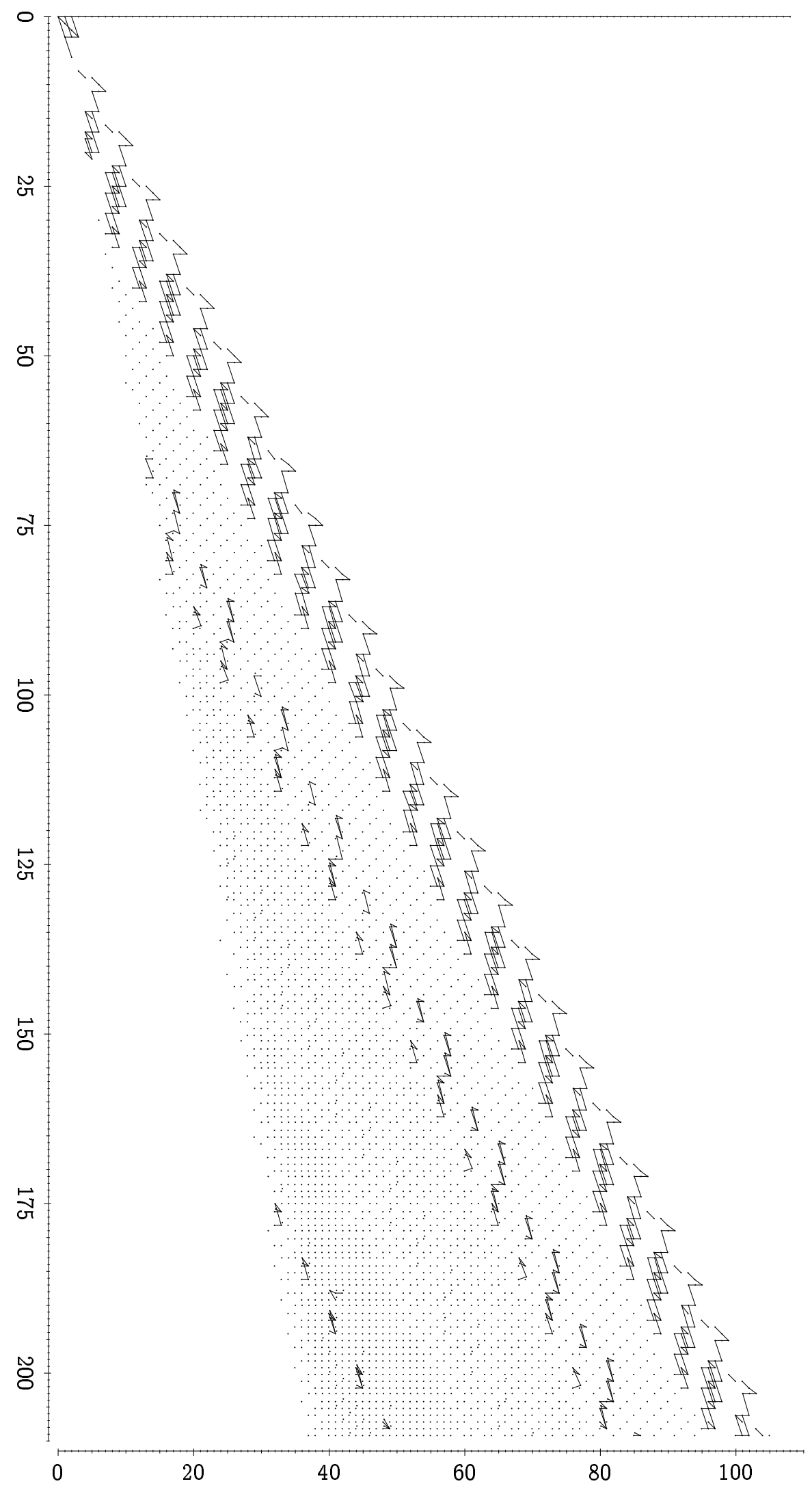

Abbildung 3.11: Bild von $\operatorname{Ext}_{A}^{*, *}\left(\mathbb{F}_{2}, \mathbb{F}_{2}\right) \rightarrow \operatorname{Ext}_{A(2)}^{*, *}\left(\mathbb{F}_{2}, \mathbb{F}_{2}\right)$ 
3 Weitere Anwendungen 


\section{Abbildungsverzeichnis}

1.1 Multiplizieren in der Milnorbasis . . . . . . . . . . . . . . . 8

$1.2 \pi_{n+k}\left(S^{n}\right)$ nach [46] . . . . . . . . . . . . . . . . . . . . . . 14

$1.3 \quad E_{2}$-Term für $\pi_{*}(H \mathbb{Z}) \ldots \ldots \ldots \ldots \ldots$

$1.4 \quad E_{2}$-Term für $\pi_{*}(S)_{(2)} \ldots \ldots \ldots \ldots \ldots \ldots \ldots \ldots$

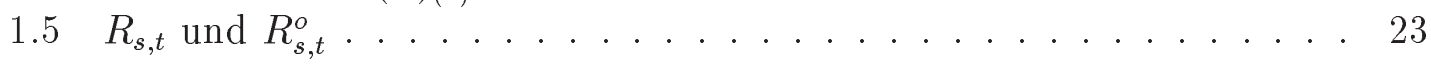

1.6 Dimension der Steenrod-Algebra . . . . . . . . . . . . . . . . . . 25

1.7 Anfang der minimalen Auflösung . . . . . . . . . . . . . 26

2.1 Lemma, obere Version . . . . . . . . . . . . . . . . . . . . 32

2.2 Lemma, untere Version . . . . . . . . . . . . . . . . . . . . . 33

2.3 Graphische Darstellung einer Profilfunktion . . . . . . . . . . . . 36

2.4 Schrittzerlegungen . . . . . . . . . . . . . . . . . . . . 38

2.5 Zerlegungsstatistik bei unterer Anwendung . . . . . . . . . . . . . . . 44

2.6 Anwendbarkeit der unteren Version . . . . . . . . . . . . . . . . . . 45

2.7 Anwendbarkeit der oberen Version . . . . . . . . . . . . . . . . . . 47

2.8 Zerlegungsstatistik bei oberer Anwendung . . . . . . . . . . . . 50

2.9 Bild zur Wahl der Unteralgebra . . . . . . . . . . . . . . . . . 54

2.10 Ext-Panorama für die Sphäre, $p=2 \ldots \ldots \ldots 6$

2.11 Ext über $A(2)$ für $p=2 \ldots \ldots \ldots \ldots$. . . . . . . . . . . . . . . . . . . . . . . . . 57

2.12 Dimension von $\operatorname{Ext}_{A}^{s, t}\left(\mathbb{F}_{2}, \mathbb{F}_{2}\right) \ldots \ldots \ldots \ldots \ldots$

2.13 Rechenzeit . . . . . . . . . . . . . . . . 58

2.14 Wachstum der minimalen Auflösung . . . . . . . . . . . . . 59

2.15 Datenmenge . . . . . . . . . . . . . . . . . 59

$3.1 \quad E^{2}$-Term für $\Sigma^{-1} \mathbb{R} P^{2} \ldots \ldots \ldots \ldots$. . . . . . . . . . . . . 66

$3.2 \quad E^{2}$-Term für den Fragezeichen-Komplex . . . . . . . . . . . . . . . 67

$3.3 \quad E^{2}$-Term für $A_{1} \ldots \ldots \ldots \ldots \ldots$. . . . . . . . . . . . 68

$3.4 \quad E^{2}$-Term für $A_{2} \ldots \ldots \ldots \ldots \ldots \ldots \ldots$

3.5 $E^{2}$-Term für $\Sigma^{3} B_{1} \ldots \ldots \ldots \ldots$. . . . . . . . . . . . . 70

$3.6 \quad E^{2}$-Term für $\Sigma^{7} B_{2} \ldots \ldots \ldots \ldots \ldots \ldots$. . . . . . . . . . . . . . . . . . . . . 71

3.7 Wurzelinvarianten in $(n, k-n)$-Koordinaten . . . . . . . . 73

3.8 Wurzelinvarianten in $(k-n, s)$-Koordinaten . . . . . . . . . . 73

3.9 Bild von $\mathrm{Sq}^{0}: \operatorname{Ext}_{A}^{* * *}\left(\mathbb{F}_{2}, \mathbb{F}_{2}\right) \rightarrow \operatorname{Ext}_{A}^{*, 2 *}\left(\mathbb{F}_{2}, \mathbb{F}_{2}\right) \ldots \ldots . \ldots 76$

$3.10 E_{2}$-Term für $\pi_{*} \mathrm{ko}_{(2)}$ mit Hurewicz-Bild . . . . . . . . . . . . . . 79

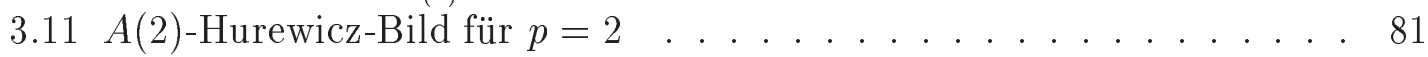


Abbildungsverzeichnis 


\section{Literaturverzeichnis}

[1] J. F. Adams. On the groups $J(X)$. IV. Topology, 5:21-71, 1966.

[2] J. F. Adams. Stable homotopy and generalised homology. University of Chicago Press, Chicago, Ill., 1974. Chicago Lectures in Mathematics.

[3] J. F. Adams and H. R. Margolis. Sub-Hopf-algebras of the Steenrod algebra. Proc. Cambridge Philos. Soc., 76:45-52, 1974.

[4] Alejandro Adem and R. James Milgram. Cohomology of finite groups. SpringerVerlag, Berlin, 1994.

[5] Donald W. Anderson and Donald M. Davis. A vanishing theorem in homological algebra. Comment. Math. Helv., 48:318-327, 1973.

[6] M. Ando, M. Hopkins, and N. P. Strickland. Elliptic spectra, the Witten genus and the Theorem of the cube. Preprint.

[7] A. K. Bousfield. A classification of $K$-local spectra. J. Pure Appl. Algebra, 66(2):121-163, 1990.

[8] A. K. Bousfield, E. B. Curtis, D. M. Kan, D. G. Quillen, D. L. Rector, and J. W. Schlesinger. The mod $-p$ lower central series and the Adams spectral sequence. Topology, 5:331-342, 1966.

[9] R. R. Bruner, J. P. May, J. E. McClure, and M. Steinberger. $H_{\infty}$ ring spectra and their applications. Springer-Verlag, Berlin, 1986.

[10] Robert Bruner and John Greenlees. The Bredon-Löffler conjecture. Experiment. Math., 4(4):289-297, 1995.

[11] Robert R. Bruner. Internetseite. http://www.math.wayne.edu/ rrb/cohom.

[12] Robert R. Bruner. Ext in the nineties. In Algebraic topology (Oaxtepec, 1991), pages 71-90. Amer. Math. Soc., Providence, RI, 1993.

[13] Robert R. Bruner. Some remarks on the root invariant. In Stable and unstable homotopy (Toronto, ON, 1996), pages 31-37. Amer. Math. Soc., Providence, RI, 1998.

[14] Robert R. Bruner. Some root invariants and Steenrod operations in $\operatorname{Ext}_{A}\left(F_{2}, F_{2}\right)$. In Homotopy theory via algebraic geometry and group representations (Evanston, IL, 1997), pages 27-33. Amer. Math. Soc., Providence, RI, 1998. 
[15] Robert R. Bruner. A Yoneda description of the Steenrod operations. Proc. Symp. Pure Math. 63, 1998.

[16] Donald M. Davis and Mark Mahowald. $v_{1^{-}}$and $v_{2}$-periodicity in stable homotopy theory. Amer. J. Math., 103(4):615-659, 1981.

[17] W. G. Dwyer and C. W. Wilkerson. A new finite loop space at the prime two. J. Amer. Math. Soc., 6(1):37-64, 1993.

[18] A. D. Elmendorf, I. Kriz, M. A. Mandell, and J. P. May. Rings, modules, and algebras in stable homotopy theory. American Mathematical Society, Providence, RI, 1997. With an appendix by M. Cole.

[19] M. Hopkins. Topological modular forms, the Witten genus, and the Theorem of the cube. In Proceedings of the International Congress of Mathematicians, pages 554-565. Birkhäuser, Basel, 1994.

[20] M. Hopkins and M. Mahowald. From elliptic curves to homotopy theory. A preliminary report, 1998.

[21] Intel Corporation. Pentium Processor Family Developer's Manual.

[22] L. G. Lewis, Jr., J. P. May, M. Steinberger, and J. E. McClure. Equivariant stable homotopy theory. Springer-Verlag, Berlin, 1986. With contributions by J. E. McClure.

[23] Elon L. Lima. The Spanier-Whitehead duality in new homotopy categories. Summa Brasil. Math., 4:91-148 (1959), 1959.

[24] W. H. Lin, D. M. Davis, M. E. Mahowald, and J. F. Adams. Calculation of Lin's Ext groups. Math. Proc. Cambridge Philos. Soc., 87(3):459-469, 1980.

[25] A. Liulevicius. Coalgebras, resolutions, and the computer. Math. Algorithms, $1: 4-11,1966$.

[26] M. Mahowald and R. James Milgram. Operations which detect $\mathrm{Sq}^{4}$ in connective K-theory and their applications. Quart. J. Math. Oxford Ser. (2), 27(108):415432, 1976.

[27] Mark Mahowald. The metastable homotopy of $S^{n}$. American Mathematical Society, Providence, R.I., 1967.

[28] Mark E. Mahowald and Douglas C. Ravenel. The root invariant in homotopy theory. Topology, 32(4):865-898, 1993.

[29] H. R. Margolis. Spectra and the Steenrod algebra. North-Holland Publishing Co., Amsterdam, 1983. Modules over the Steenrod algebra and the stable homotopy category.

[30] J. P. May. The cohomology of restricted Lie algebras and of Hopf algebras. J. Algebra, 3:123-146, 1966. 
[31] J. Peter May. A general algebraic approach to Steenrod operations. In The Steenrod Algebra and its Applications (Proc. Conf. to Celebrate N. E. Steenrod's Sixtieth Birthday, Battelle Memorial Inst., Columbus, Ohio, 1970), pages 153231. Springer, Berlin, 1970.

[32] John McCleary. A user's guide to spectral sequences. Cambridge University Press, Cambridge, second edition, 2001.

[33] H. R. Miller and C. Wilkerson. Vanishing lines for modules over the Steenrod algebra. J. Pure Appl. Algebra, 22:293-307, 1981.

[34] John Milnor. The Steenrod algebra and its dual. Ann. of Math. (2), 67:150-171, 1958.

[35] John W. Milnor and John C. Moore. On the structure of Hopf algebras. Ann. of Math. (2), 81:211-264, 1965.

[36] Stephen A. Mitchell. Finite complexes with $A(n)$-free cohomology. Topology, 24(2):227-246, 1985.

[37] Robert E. Mosher and Martin C. Tangora. Cohomology operations and applications in homotopy theory. Harper \& Row Publishers, New York, 1968.

[38] C. Nassau. Internetseite. http://www.math.uni-frankfurt.de/ nassau/Ext2.

[39] Douglas C. Ravenel. Complex cobordism and stable homotopy groups of spheres. Academic Press Inc., Orlando, FL, 1986.

[40] Douglas C. Ravenel. Nilpotence and periodicity in stable homotopy theory. Princeton University Press, Princeton, NJ, 1992. Appendix C by Jeff Smith.

[41] Nobuo Shimada and Akira Iwai. On the cohomology of some Hopf algebras. Nagoya Math. J., 30:103-111, 1967.

[42] N. E. Steenrod. Cohomology operations. Princeton University Press, Princeton, N.J., 1962.

[43] R. M. Switzer. Algebraic Topology - Homotopy and Homology. Springer Verlag, Berlin and New York, 1975.

[44] Martin C. Tangora. On the cohomology of the Steenrod algebra. Math. Z., 116:18-64, 1970.

[45] Martin C. Tangora. Computing the homology of the lambda algebra. Mem. Amer. Math. Soc., 58(337):v+163, 1985.

[46] Hirosi Toda. Composition methods in homotopy groups of spheres. Princeton University Press, Princeton, N.J., 1962.

[47] Edward Witten. The index of the Dirac operator in loop space. In Elliptic curves and modular forms in algebraic topology (Princeton, NJ, 1986), pages 161-181. Springer, Berlin, 1988. 
Literaturverzeichnis 


\section{Danksagung}

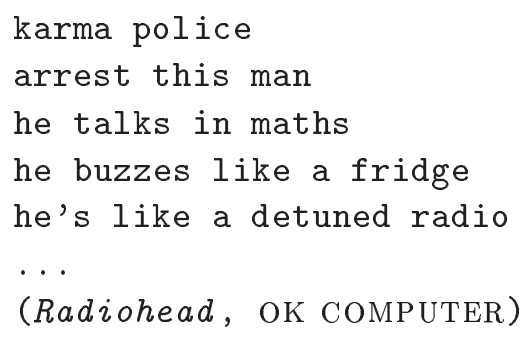

Zuerst gilt mein Dank Prof. Kultze für die unproblematische und großzügige Betreuung. Damit hat sich eine Tradition, die mit meiner Diplomarbeit begann, glücklich fortgesetzt. Zu danken ist auch Bob Bruner, für eine Reihe ermutigender E-Mails, die im Laufe der Jahre eintrudelten, und für seinen meist offenen E-Briefkasten.

Matthias Schork hat freundlicherweise Teile einer Frühfassung Korrektur gelesen. Meine tiefsitzende Dudenskepsis machte dies vermutlich zu einem etwas frustrierenden Unterfangen, was wohl meine Dankesschuld verdoppelt. Dank gilt außerdem Prof. Ossa für das Aufspüren kleinerer Fehler. Ihm ist es auch zu Gute zu halten, daß der Algorithmus jetzt etwas expliziter beschrieben wird, als es zunächst von mir geplant war.

Um eine schöne Tradition fortzuführen gilt mein Dank natürlich auch den mittlerweile zumeist ehemaligen - An- und Zugehörigen der Arbeitsgruppe 8:1 von und um Prof. Weidmann, nämlich mindestens Holger Altenhofen, Dirk Buschmann, David Damanik, Jacqueline Habash, Steffen Klassert, Frank Kleespies, Daniel Lenz, Bernd Metzger, Andreas U. Schmidt, Matthias Schork, Peter Stollmann sowie (last, not least) Andreas Weng, geb. Hoffmann, deretwegen ich meinen Kaffee all die Jahre nicht alleine trinken mußte. Gott vergelt's.

Schließlich bleibt nur noch Dank an Douglas Ravenel für das Schreiben von [39]. Ohne diese Sphären- oder Sirenenmusik, die nicht so heißen durfte [und mittlerweile, wie's scheint, auch nicht mehr so heißen soll], wäre zumindest mein akademisches Leben anders verlaufen.

Finally, I apologize for not writing this thing in English. (No joke) 
cn@maths: / > ls -1 fu**

lrwxrwxrwx $1 \mathrm{cn}$ idiots 0 Feb 292002 future -> /dev/null cn@maths: $\sim />$ 

Logos Verlag Berlin

ISBN 3-89722-881-5 